Portland State University

PDXScholar

Civil and Environmental Engineering Master's

Project Reports

Civil and Environmental Engineering

Winter 2021

\title{
Small Scale Plasma Gasification of Municipal Solid Waste
}

Keith Male

Portland State University

Follow this and additional works at: https://pdxscholar.library.pdx.edu/cengin_gradprojects

Part of the Civil and Environmental Engineering Commons Let us know how access to this document benefits you.

Recommended Citation

Male, Keith, "Small Scale Plasma Gasification of Municipal Solid Waste" (2021). Civil and Environmental Engineering Master's Project Reports. 55.

https://doi.org/10.15760/CCEMP.54

This Project is brought to you for free and open access. It has been accepted for inclusion in Civil and Environmental Engineering Master's Project Reports by an authorized administrator of PDXScholar. Please contact us if we can make this document more accessible: pdxscholar@pdx.edu. 


\title{
KEITH MALE
}

A research project report submitted in partial fulfillment of the requirement for the degree of

\author{
MASTER OF SCIENCE \\ IN \\ CIVIL AND ENVIRONMENTAL ENGINEERING
}

Project Advisor:

Dr. Gwynn R. Johnson

P Portland $\int_{\text {UNIVERSITY }}^{\text {State }}$

Portland State University

(C)2021 


\section{ABSTRACT}

The goal of this project was to develop a prototype plasma gasification system to treat municipal solid waste (MSW) with minimal regulated emissions in a footprint small enough to be transported for a wide range of applicability. This project started with a review of current MSW treatment methods and of the emissions produced from thermal breakdown of MSW. A review of air quality regulations and emission control technologies was then used to select the emission control systems to be used for a small-scale plasma gasification system.

This plasma gasification system began with a plasma torch and cooling system being designed, built, and tested with various electrode materials and designs. The torch was tested using compressed air, nitrogen, and a mixture of argon and hydrogen. Tungsten was chosen for the positive electrode and copper for the negative electrode, with compressed nitrogen as the process gas. A gasification chamber was designed and built to handle the molten material and act as a platform for the torch-centering device, exhaust port, and viewing window. Several emission control systems were built based on expected pollutants of MSW decomposition. These controls include a particle separator, catalytic converter and a packed column scrubber. Gas and atmospheric sensors were then programmed and installed in the exhaust gas stream.

This prototype plasma gasification system was built with the intent of testing various materials one at a time and measuring the emissions produced. This data would then have been used to modify and improve the emission controls used to eliminate or capture any contaminants in the syngas, with the intent of having the syngas be a mixture of only hydrogen and carbon monoxide. Later additions would include a fuel cell to be used with the hydrogen, a heat exchanger and turbine to recover energy from the heat produced, and material recovery systems for the contaminants detected. 


\section{TABLE OF CONTENTS}

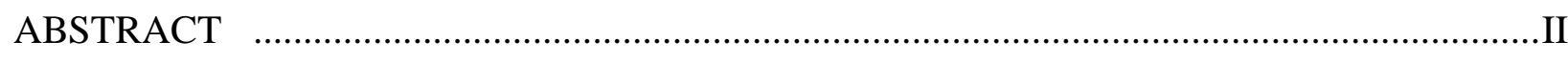

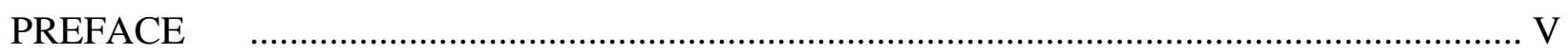

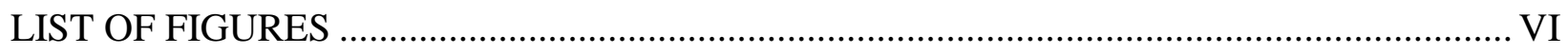

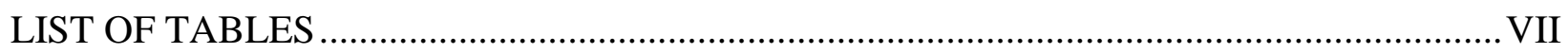

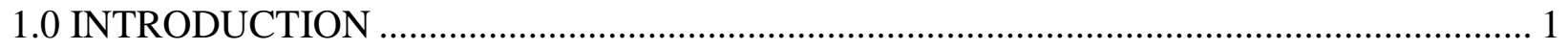

2.0 CURRENT MUNICIPAL WASTE MANAGEMENT METHODS ..................................... 4

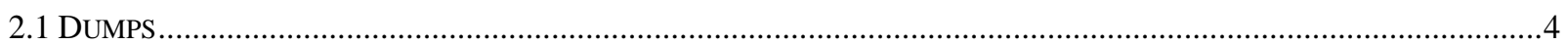

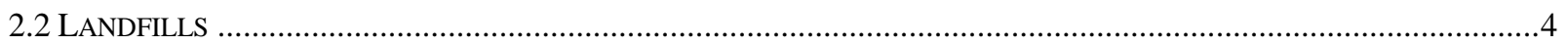

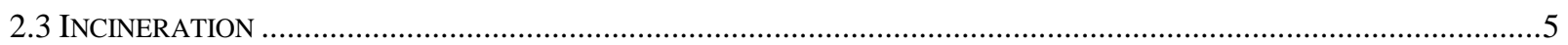

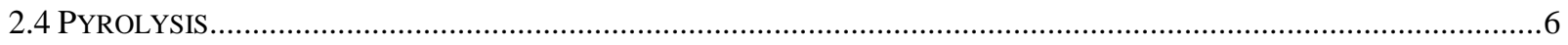

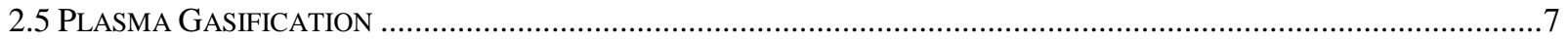

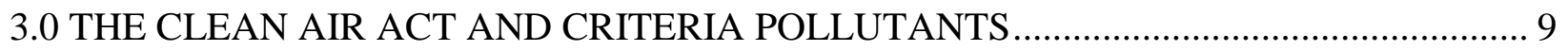

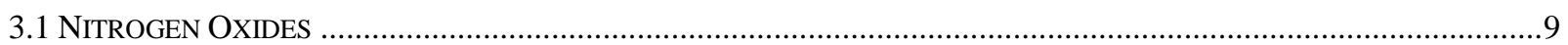

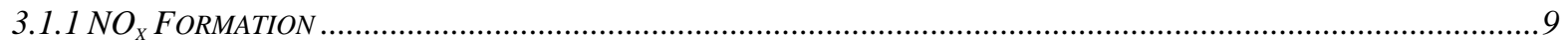

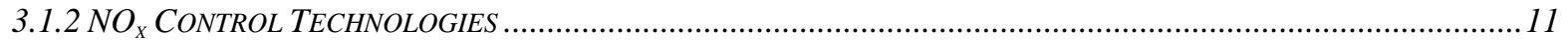

3.1.2.1 Selective non-catalytic reduction (SCNR) .............................................................................................. 11

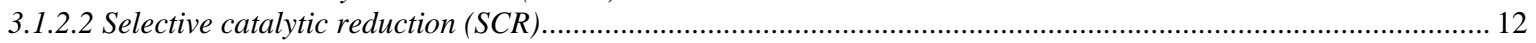

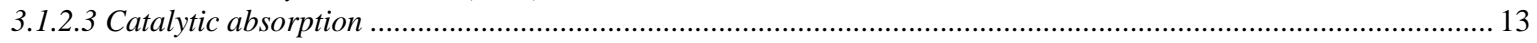

3.1.2.4 Low temperature oxidation with absorption ……….................................................................................... 14

3.1.2.5 Corona-induced plasma ..................................................................................................................... 14

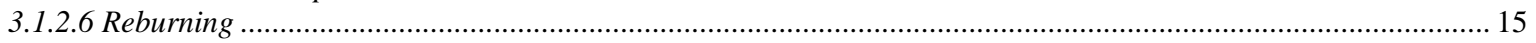

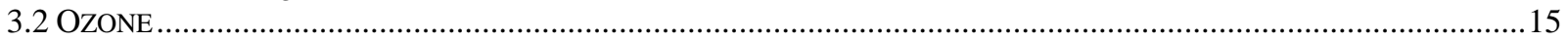

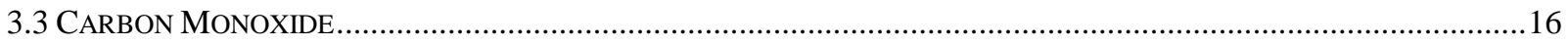

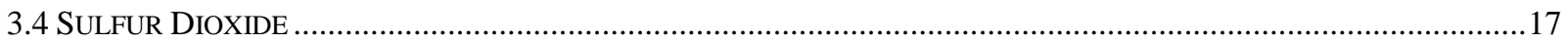

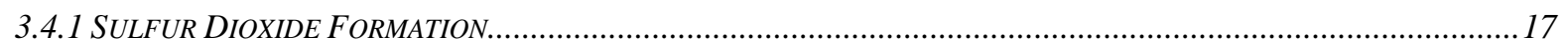

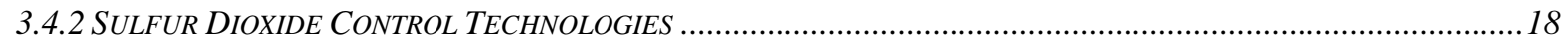

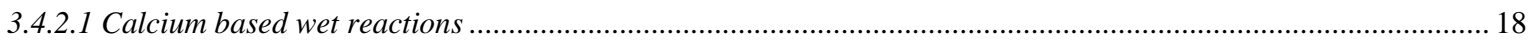

3.4.2.2 Calcium based dry reactions ………………….............................................................................. 19

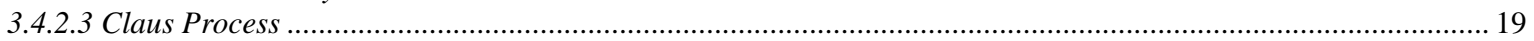

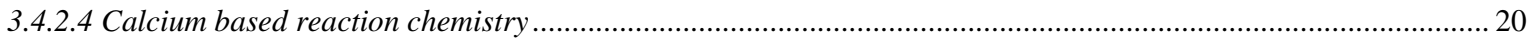

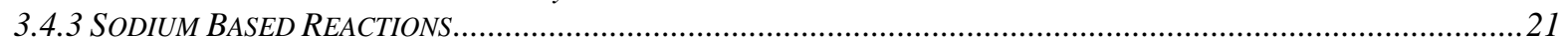

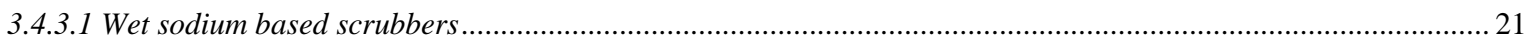

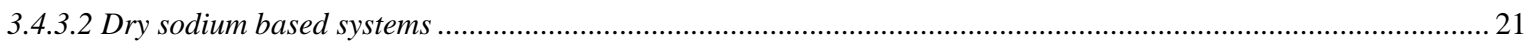

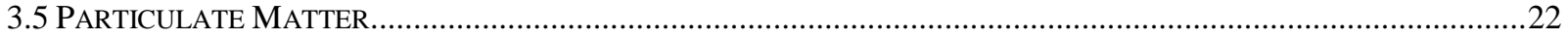

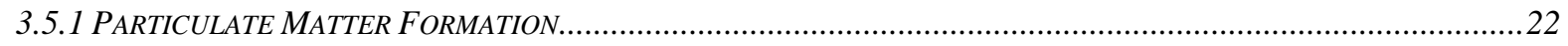

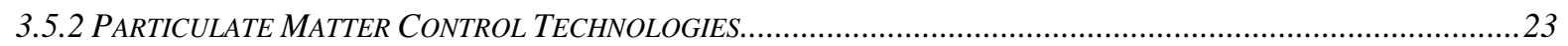

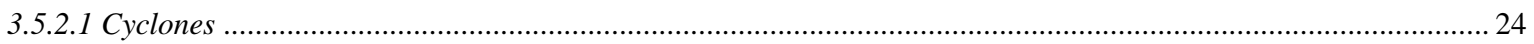

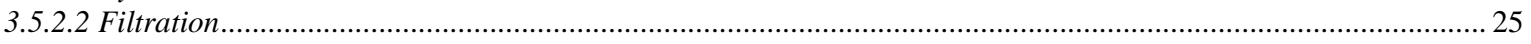

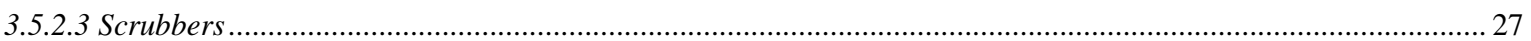

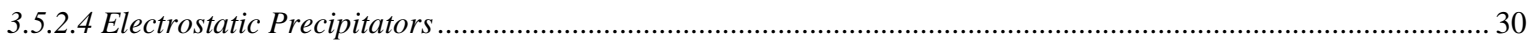

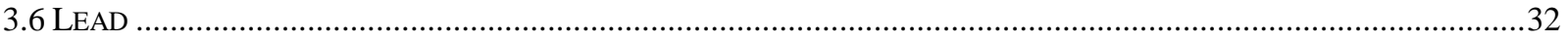

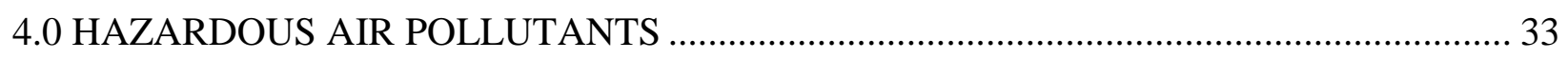

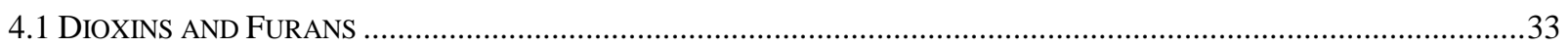

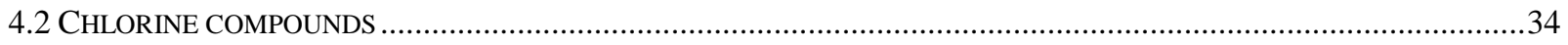

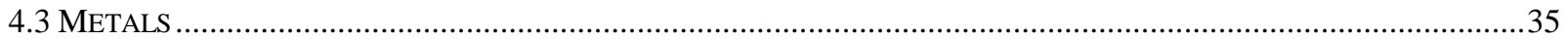

5.0 PROTOTYPE PLASMA GASIFICATION SYSTEM AND DESIGN COMPONENTS ..... 36

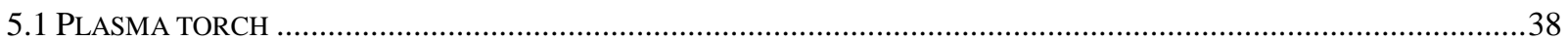

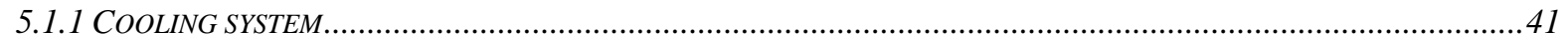

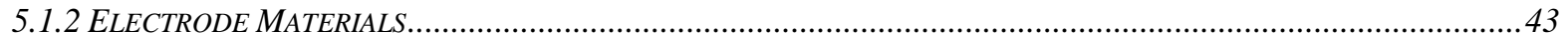




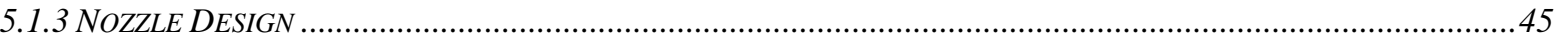

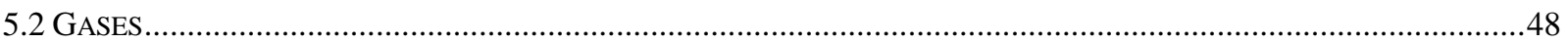

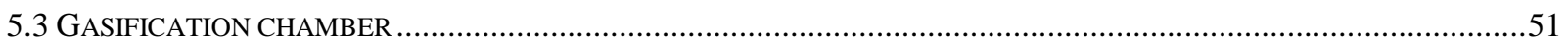

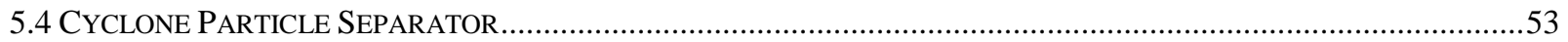

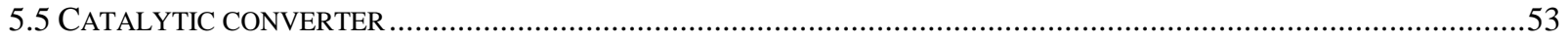

5.6 SCRUBBER

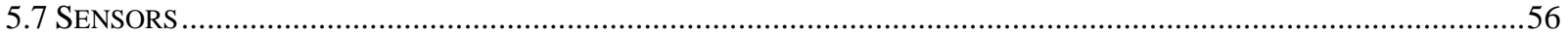

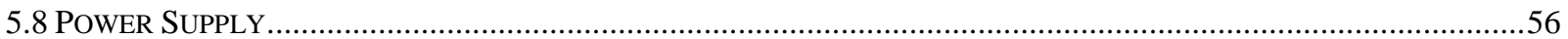

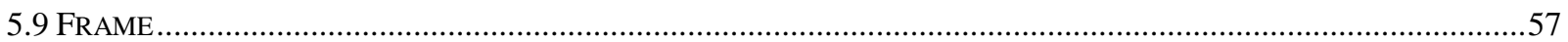

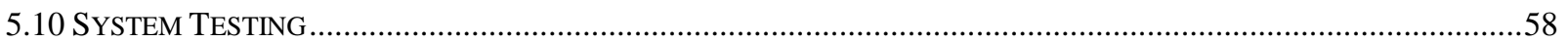

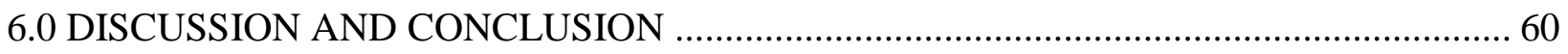

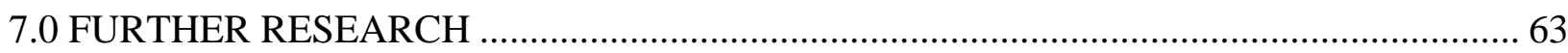

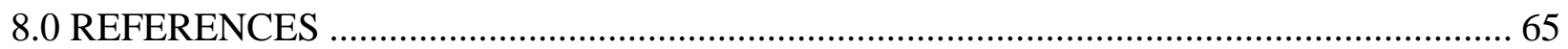




\section{PREFACE}

This project was started with the intent of testing the gasification of various feedstocks in the Contaminant Transport and Chemistry Lab in the Department of Civil and Environmental Engineering at Portland State University. The plasma torch, gasification chamber, emissions control systems and sensors, and frame were all built with preliminary testing conducted between the summer of 2019 and March 2020. The next steps in the process included further testing and optimization of the prototype beginning in spring 2020. During March 2020, the COVID-19 virus caused much of the United States and of the world to shut down and restrict access to public spaces. Portland State followed similar restrictions, preventing all extended access to the university's lab spaces. This restriction halted all testing and access to any on-campus shops (and labs) preventing access to necessary equipment to manufacture/produce additional or modified parts necessary to optimize the prototype plasma torch. Furthermore, while preliminary studies included sensing gas-phase emissions, those preliminary tests indicated the system would require flow controls. Further research recommendations are provided as the final chapter of this report. 


\section{LIST OF FIGURES}

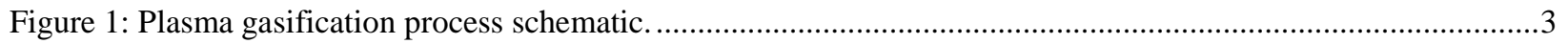

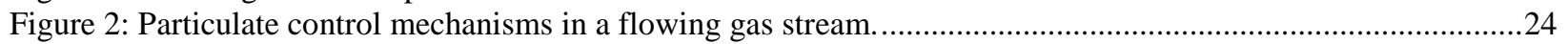

Figure 3: Particle trajectories in a cyclone particle separator of 10, 50 and 100 micron particles.............................25

Figure 4: Impingement spray scrubber internal components and capture mechanism. ............................................29

Figure 5: Venturi scrubber design possibilities, components and capture mechanism ..........................................30

Figure 6: Electrostatic precipitator charge production and particle collection mechanism. ......................................31

Figure 7: Schematic of plasma gasification system components, with inputs and outputs.........................................37

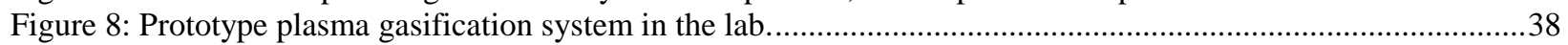

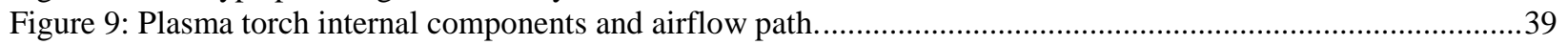

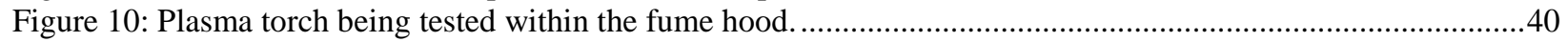

Figure 11: Coolant system with radiator, fan, pump, power supply, and coolant tubes. ...........................................42

Figure 12: Coolant jacket for the plasma torch...................................................................................................

Figure 13: Graphite electrode wear on both positive and negative electrodes ........................................................43

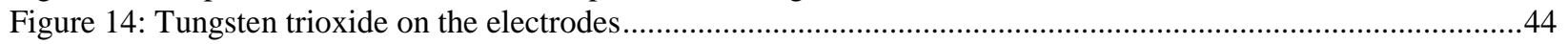

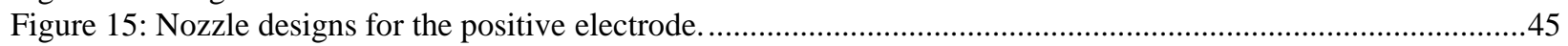

Figure 16: Copper 0.040 in. diameter nozzle after the testing ............................................................................46

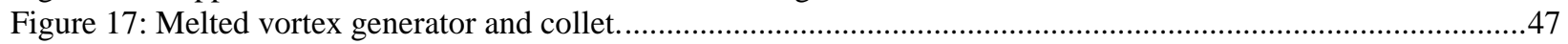

Figure 18: Torch centering device with glass-filled nylon screws ...................................................................48

Figure 19: Specific enthalpy for different monatomic and molecular gases as a function of temperature.................49

Figure 20: Equilibrium composition for argon gas (left) and nitrogen (right) as a function of temperature. .............49

Figure 21: Thermal conductivity for $\mathrm{Ar} / \mathrm{H} 2$ mixtures as a function of temperature ...............................................50

Figure 22: Plasma produced with compressed nitrogen (left) and argon/hydrogen mixture (right). ..........................51

Figure 23: Gasification chamber being filled with refractory cement. ......................................................................52

Figure 24: Gasification chamber filled with test material................................................................................53

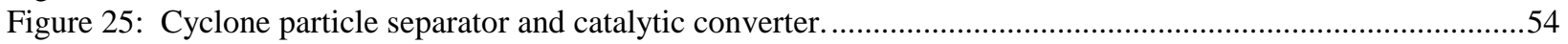

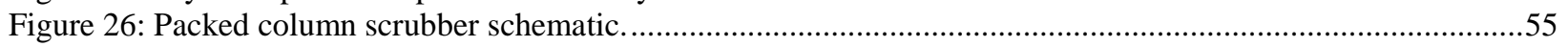

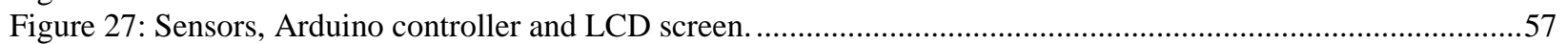

Figure 28: Plasma system prototype frame front (left) and back (right)..............................................................58

Figure 29: Molten glass and metals inside the gasification chamber.....................................................................59 


\section{LIST OF TABLES}

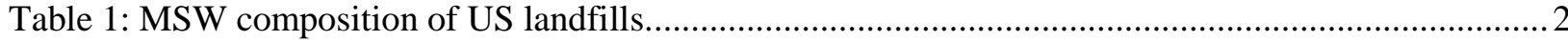




\subsection{INTRODUCTION}

Global municipal solid waste (MSW) production was estimated to be 2.01 billion tons annually for the year $2016^{[1]}$. This rate is expected to increase to 3.40 billion tons by 2050 if humans continue with the current material based economy. The degree of collection and disposal of this waste depends on the country's income level. Lower income countries tend to have between $10.6-55 \%$ collection rates compared to $76-100 \%$ collection for higher income countries ${ }^{[1]}$. Countries with $100 \%$ collection rates do not include litter, which is waste that falls off the collection vehicles or blows away in the wind and waste that never makes it to a collection container. Waste that is not collected often goes directly into the environment via runoff from streets or is simply thrown into piles on the edges of villages and towns.

Disposal methods are similarly influenced by income level, with lower income countries sending more of the collected waste to open dumps and landfills and higher income countries using more expensive technologies such as recycling, composting and waste to energy (WTE) plants. The majority of the world uses landfills as the main disposal methods as it is generally the lowest immediate cost.

In the United States as of 2006, 54\% of the collected MSW goes to landfills, $24 \%$ of waste is recycled, $8 \%$ goes to be composted and the remaining $14 \%$ is converted to energy (WTE) ${ }^{[1]}$. This means that $54 \%$ of the waste in the US is not utilized. Within this $54 \%$, there are many materials (Table 1) that can be composted, separated and brought back into the economy, or recovered for energy content. Glass, metals, and inorganic wastes can be recovered, while paper and paperboard, yard trimmings, food and wood can all be composted and any of the other materials that cannot be recovered can be processed to recover the energy content. The effort spent gathering and processing these materials from the earth is wasted if these materials end up in a landfill instead of being reused. This is cradle to grave design, where products are manufactured, used and then discarded as waste, and was popular when there were plenty of resources available and human impact on the planet was low. Items that follow cradle to cradle design are manufactured, used and repaired for as long as possible, then disassembled for material recovery. With an ever expanding population, anthropogenic-induced climate change and widespread pollution, there must be a cradle to cradle mindset to reduce the total waste produced. 
Table 1: MSW composition of US landfills.

\begin{tabular}{|l|c|}
\hline \multicolumn{1}{|c|}{ Material group } & $\begin{array}{c}\text { \% mix of } \\
\text { MSW* }\end{array}$ \\
\hline Glass & 4.9 \\
\hline Metals & 9.9 \\
\hline Paper and paperboard & 13.1 \\
\hline Yard Trimmings & 6.2 \\
\hline Food & 21.9 \\
\hline Wood & 8.7 \\
\hline Textiles & 8.0 \\
\hline Misc. Inorganic Wastes & 2.3 \\
\hline Rubber and Leather & 3.5 \\
\hline Plastics & 19.2 \\
\hline Other & 2.1 \\
\hline
\end{tabular}

*Percentages listed on epa.gov for total MSW landfilled in 2017.

The transportation of waste from the source to the destination is not insignificant in terms of impact on the environment compared to the impact of collection and treatment. Waste collection vehicles have a route that the trucks follow and stop for anywhere from 10 to 17 seconds at each house and another 11 to 13 seconds between each stop ${ }^{[2]}$. Internal combustion engines use the most amount of fuel during acceleration and this constant deceleration and acceleration of waste collection vehicles with heavy loads and diesel engines lead to fuel efficiencies of between 1.4 and 3.3 miles per gallon $(\mathrm{mpg})^{[2]}$. These vehicles then travel to either landfills, transfer stations, waste to energy plants or material recovery facilities and spend anywhere from 7 to 22 minutes unloading, where vehicles with larger loads spend more time unloading, often with their diesel engines sitting idle for the duration of that time ${ }^{[2]}$. Landfills commonly utilize bulldozers, front loaders, and other heavy machinery run by diesel engines, which are considered non-road compression-ignition engines and have less stringent emission controls than that of on-road vehicles, to move the MSW in formation to seal off each layer ${ }^{[3][4]}$. All of these low efficiency diesel vehicles lead to a significant amount of emissions and air pollution dealing with the transportation and organization of waste. A review of the literature reveals that the specific amount of emissions from waste transportation vehicles may not be well quantified, however many models exist to attempt to quantify the amount ${ }^{[2],[5],[6],[7]}$. A simple way to reduce these emissions is to optimize the collection and transportation routes of the vehicles, which can also greatly reduce the associated costs ${ }^{[8]}$. 
An overview of waste treatment technologies is provided to show how plasma gasification applied as a waste treatment/materials recovery option has the potential to greatly reduce the emissions and other environmental damage caused by antiquated waste management methods. This project investigates whether a small, low cost plasma gasification system can produce close to zero emissions with the proper controls. In order to explain the reasoning for the particular emission control systems chosen in this project, a background of air quality regulations is provided. A review of the technologies that are currently in use is also provided to show the relative costs and benefits of each technology and reasons why each may or may not be viable in a small-scale system. With the background in air quality controls, a more detailed description of the plasma gasification system used in this project can be appreciated. The plasma gasification system designed, built, and used for this project includes a gasification chamber, particle separator, catalytic converter, packed column scrubber and a set of sensors to test pollutants passing the emission controls (Figure 1). The tests of the components in this system are discussed, along with recommendations for improvement.

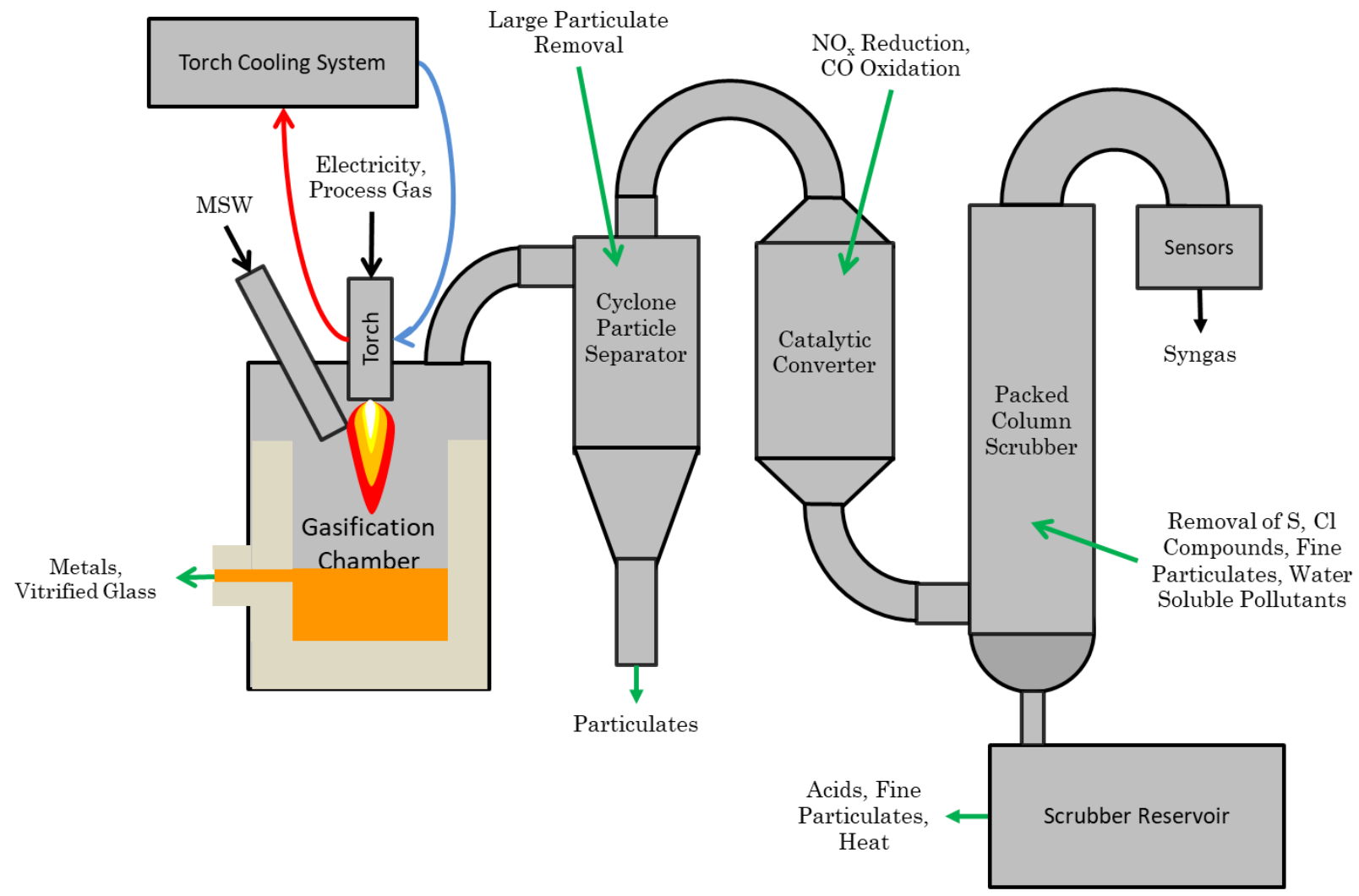

Figure 1: Plasma gasification process schematic. 


\subsection{CURRENT MUNICIPAL WASTE MANAGEMENT METHODS}

\section{$\underline{2.1 \text { Dumps }}$}

The simplest, cheapest method of waste disposal is an open dump, which involves piling waste on the ground near the source. Open dumps are sometimes burned to reduce volume and often catch fire as combustibles decompose, producing smoke along with methane gas upon decomposition of organic waste. These dumps are commonly not bottom sealed from chemicals of decomposition leeching into the surrounding water bodies, which are usually close to the communities that fill the dump. Open dumps allow release of the chemicals and decomposition byproducts into the environment. These chemicals often cause health and safety issues for the nearby communities. Higher income countries typically prohibit open dumps. There are many lower income regions of the world where open dumps still exist.

\section{$\underline{2.2 \text { Landfills }}$}

Engineered landfills, a more sophisticated type of dump, are typically designed with a bottom lining system and covered or topped every day to minimize pollution released. In European and American landfills, the ground is typically covered with an impermeable or semi-impermeable geomembrane followed by a geotextile and a system of leachate collection pipes laid on top of the geotextile ${ }^{[9]}$. The leachate collection pipes are then covered in a mineral barrier and finally a drainage layer to allow leachates to reach the pipes ${ }^{[9]}$. The waste is then laid on top of this lining system. The cover is typically made up of the same type of liner placed on top of the waste, with additional soil on top of the cover ${ }^{[10]}$. Gas collection pipes are installed to capture the gases produced within the landfill. These gases are collected for use as fuels and typically are composed of $45-55 \%$ methane and $40-50 \%$ carbon dioxide ${ }^{[11]}$. Some landfills choose to burn the methane with flares and release methane combustion products directly into the environment. Landfills do occasionally catch fire during gas extraction or from sparking of equipment ${ }^{[11]}$. Aerobic microbial reactions can also increase the temperature and ignite gases within the landfill $^{[11]}$.

Landfill coverings are meant to seal in the gases produced by decomposition of organic matter and prevent rainwater from entering. Landfill linings are designed to prevent liquids draining from the waste to reach the soil or the groundwater; however the linings are only 
designed to last so many years. The linings can fail through erosion, freeze-thaw cycling, wetdry cycling or subsidence of the soil below at some point, which will allow leachate to be released $^{[11]}$.

The majority of leachates from landfills are released from precipitation infiltrating the landfill and leaking through the liner. One study found that $42.76 \%$ of the total precipitation leaked through the Ano Liosia landfill of the Attica region of Greece ${ }^{[12]}$. The leachate from this study found high levels of ammonia $\left(\mathrm{NH}_{3}\right)$, phosphate ions $\left(\mathrm{PO}_{4}{ }^{3-}\right)$, sulfate ions $\left(\mathrm{SO}_{4}{ }^{2-}\right)$, chloride ions $\left(\mathrm{Cl}^{-}\right)$, potassium ions $\left(\mathrm{K}^{+}\right)$, along with iron and lead ions in the groundwater close to the landfill. This study did not test for hazardous industrial chemicals, as there is not much industry in that area of Greece.

The landfill studied in Greece also had high chemical oxygen demand (COD) and a relatively low biological oxygen demand (BOD), with a relatively low ratio of BOD/COD. This low BOD/COD ratio suggests that the organic matter in the landfill is not easily biodegradable ${ }^{[12]}$. In 1991, an archaeologist, Dr. William Rathje at the University of Arizona, worked on "The Garbage Project" to determine the archaeology of contemporary landfills and found "such preserved perishables as heads of lettuce, Kaiser rolls, hot dogs, corncobs with their kernels intact, guacamole, and literally tons of datable, readable newspapers",[13]. If guacamole can be found after years in a landfill without major decomposition, then that organic matter in landfills is not doing what organic matter is supposed to with the design of current landfills. Dr. Rathje's study analyzed landfills in Arizona, which does not receive as much precipitation as in many other parts of the world where leachate would be a greater issue.

\section{$\underline{2.3 \text { Incineration }}$}

Incinerators for MSW were first built in the US in 1885 as the first level of technology above landfills $^{[14]}$. This process involves continuously feeding the waste into an incinerator with waste serving as the fuel source. The trash is burned in a chamber with air continuously injected, allowing for combustion and the high temperature chemical reactions that can form polychlorinated dibenzo-p-dioxins (PCDDs) and polychlorinated dibenzofurans (PCDFs) and other pollutants. The emissions from MSW incineration often include carbon dioxide, methane, nitrogen oxides, PCDDs, PCDFs, particulate matter including heavy metals, volatile organic compounds (VOCs), and any byproducts formed from the multitude of materials that make up 
$\mathrm{MSW}^{[15]}$. These systems commonly use air as the source of oxygen for combustion so there is little control over the reactions that form many of the emissions produced during combustion. The byproducts of this process include substantial atmospheric pollution and $a h^{[15]}$. The ash produced is commonly composed of bottom ash and fly ash, where fly ash includes the fine particles that remain airborne. As MSW can contain anything people throw in the trash, there is a wide range of chemical compounds that can form, which requires extensive emissions controls at each plant. When the Clean Air Act came into effect in 1970, the existing MSW incineration facilities had to either install emission control technology or shut down ${ }^{[14]}$. In the 1990 s, the Maximum Achievable Control Technology (MACT) regulations set forth by the EPA recognized the dangers of PCDDs and mercury emissions, resulting in another round of retrofitting emission controls or shutting incineration plants down ${ }^{[14]}$.

\subsection{Pyrolysis}

One solution to reducing the emissions from incineration is to reduce the concentration of oxygen in the combustion chamber. An oxygen starved high temperature process is called pyrolysis. The byproducts of pyrolysis include a low sulfur liquid similar to fuel oil, char, a fraction of water, and gaseous emissions ${ }^{[16]}$. This process has reduced gaseous emissions as compared to traditional incineration due to the lack of air in the heated chamber. The gaseous emissions from atmospheric pressure pyrolysis include carbon monoxide, carbon dioxide, hydrogen, $C_{1}$ to $C_{7}$ hydrocarbons and small amounts of water vapor and methyl chloride ${ }^{[16]}$. The composition of the byproducts is approximately $40 \mathrm{wt} . \%$ oil, $35 \mathrm{wt} . \%$ char, $10 \%$ gases and $15 \%$ water $^{[16]}$. The oil produced from pyrolysis is typically used as fuel oil for energy production as the oils are composed of many sizes of molecules and depend highly on the MSW feed material $^{[17]}$. A comparison of pyrolysis versus incineration of certain materials found that the production of PCDDs and PCDFs is greatly reduced, yet still not eliminated with pyrolysis ${ }^{[18]}$. For example, with polyvinyl chloride, the combusted concentration of PCDD/PCDF was $4500 \mathrm{pg}$ $\mathrm{I}_{\mathrm{TEQ}} / \mathrm{g}^{*}$ while the pyrolysis concentration was $215 \mathrm{pg} \mathrm{I-TEQ} / \mathrm{g}^{[18]}$. A similar effect was seen with a sewage sludge containing a high concentration of metals which produced $1700 \mathrm{pg}$ ITEQ/g PCDD/PCDF concentration when combusted versus 232 pg I-TEQ/g PCDD/PCDF

\footnotetext{
* pico-grams PCDD/PCDF expressed as International Toxic Equivalents/gram waste material
} 
concentration through pyrolysis $^{[18]}$. The other materials studied had far lower production of PCDD/PCDFs and had similar results with a lesser percent difference. Pyrolysis does reduce the production of PCDD/PCDFs, decreasing the required emissions controls; however the process does still produce unwanted byproducts.

Pyrolysis is used more often to process bio-mass than solid waste to increase the quality of the oil produced and allow for a lower temperature chamber to be maintained. This process is only feasible for specific homogeneous feed materials, as it must be done at specific controlled temperatures depending on the feedstock ${ }^{[19]}$. This could be a suitable process for organic material that is not easily composted or as a method for bio-fuel production. The solid residues produced must still be sent to a landfill, so it is not a full treatment for waste materials ${ }^{[19]}$.

\section{$\underline{2.5 \text { Plasma Gasification }}$}

Plasma gasification is the process transmitting a high electric current through a stream of flowing gas, resulting in the stripping of electrons from the passing molecules to create a high temperature field of ionized gas ${ }^{[20]}$. When applied to waste materials, this high temperature field breaks chemical bonds and is full of radicals, electrons, ions and excited molecules that can reach temperatures in the range of many thousands of degrees Kelvin, with some reaching $10,000 \mathrm{~K}^{[20],[21]}$. At these temperatures, the inorganic material is decomposed into vitrified slag and the organic material fully decomposed into gaseous state ${ }^{[20]}$. This process occurs in a highly insulated chamber of refractory material, where the slag can form a pool and be released in a controlled manner and the gases generated can leave through an exhaust port to refining processes. The exhaust gases are mainly hydrogen and carbon monoxide, with low levels of contaminants that need to be removed ${ }^{[19]}$. This refined mixture of hydrogen and carbon monoxide can be used for fuel purposes and is often called syngas (synthesis gas).

There are several benefits of plasma gasification over combustion processes. When plasma is used as the energy source for waste decomposition, less oxygen is required to process the feedstock $^{[19]}$. This reduction in oxygen requirements allows plasma gasification to have reduced formation of $\mathrm{SO}_{2}, \mathrm{NO}_{\mathrm{x}}$ and $\mathrm{PCDD} / \mathrm{PCDFs}^{[19]}$. The volume of process gas is also lower due to reduced oxygen demand for combustion; consequently, the equipment for cleaning the exhaust gases can be smaller and less expensive ${ }^{[19]}$. The energy density of plasma is also much higher than the feed materials used in combustion processes, which can reduce the size of the 
destruction chamber ${ }^{[19]}$. With the high energy density, plasma gasification is able to crack tars and chars that are a byproduct of pyrolysis ${ }^{[19]}$. The high energy density means that any particulates or residues produced within the cleaning process can be sent back to the input to be processed $^{[19]}$.

Plasma gasification systems have smaller footprints than landfills and do not produce the associated odors of decay. This gives plasma gasification plants more locational freedom and can be spaced out to minimize waste transportation distances. Small-scale, decentralized plasma gasification systems would reduce the emissions from waste collection and allow material and energy recovery from MSW. Decentralized systems built with sorting, recycling and composting facilities placed at the source of MSW would greatly reduce the travel time for waste collection vehicles by reducing the travel time to and from their collection routes. This would also require far less real estate than landfills currently need and prevent the environmental damage commonly associated with landfills. Small modular plasma gasification systems could be stationed at landfills to mine and treat the materials buried to prevent future environmental damage, reduce the footprint of the landfill or even extend the lifetime of operation of waste management systems. Smaller scale waste treatment systems also have the benefit of reduced capital costs, which is a major hindrance to new waste treatment technologies.

This project considers treating MSW using plasma gasification on a small scale to reduce the capital cost and the carbon footprint of large-scale waste management facilities. The goal of studying plasma gasification is to eventually eliminate the need for landfills and to excavate existing landfills to restore them to their natural clean state while reclaiming or mining the landfilled materials. If the capital cost and operating costs can be sufficiently reduced, then this technology could be used even in the lower income regions of the world to dispose of the waste being sent to open dumps and landfills. In application, this process could be delivered to parts of the world with no effective waste management system. 


\subsection{THE CLEAN AIR ACT AND CRITERIA POLLUTANTS}

The Clean Air Act, passed in 1963, established federal responsibility of air pollution

control $^{[22]}$. This was mainly aimed at establishing criteria of air pollutants for the protection of public health and welfare, although the original Clean Air Act did not have specific requirements. The Clean Air Act Amendments of 1970 established several programs with these requirements and created the U.S. Environmental Protection Agency (EPA) ${ }^{[22]}$. The National Ambient Air Quality Standards (NAAQS) established criteria pollutants at two levels: primary standards to protect health, and secondary standards to protect welfare ${ }^{[22]}$. The six criteria pollutants include nitrogen dioxide $\left(\mathrm{NO}_{2}\right)$, ozone $\left(\mathrm{O}_{3}\right)$, carbon monoxide $(\mathrm{CO})$, sulfur dioxide $\left(\mathrm{SO}_{2}\right)$, particulates, and lead $(\mathrm{Pb})^{[22]}$. Volatile organic compounds (VOCs) were not included as a criteria pollutant, as VOCs and nitrogen oxides are precursors to ozone, which is one of the criteria pollutants $^{[22]}$. There are also a huge number of volatile organic compounds, so regulating them as a single group is impossible. Another program, the New Source Performance Standards (NSPS), required any new source of air pollution to apply effective air pollution controls and any modification to existing sources to upgrade air pollution control equipment ${ }^{[22]}$. The NSPS allowed existing sources to be "grandfathered" in without retrofitting their pollution controls ${ }^{[22]}$. A third major program was the National Emission Standards for Hazardous Air Pollutants (NESHAP). This program, NESHAP, was too cumbersome for the EPA to effectively implement, as there were over a hundred hazardous chemicals to be regulated, all requiring research and testing to determine acceptable levels for human and environmental health ${ }^{[22]}$. Due to this lack of research, NESHAP standards were only initially widespread for a small number of hazardous pollutants.

\section{$\underline{3.1 \text { Nitrogen Oxides }}$}

\section{$\underline{3.1 .1 \mathrm{NO}_{x}}$ Formation}

Nitrogen oxides include many different species, with nitrogen dioxide $\left(\mathrm{NO}_{2}\right)$ and nitrogen oxide (NO) being of primary concern. Nitrogen dioxide is one of the six criteria pollutants, and nitrogen oxide is involved in both $\mathrm{NO}_{2}$ and ozone formation. The main mechanisms for $\mathrm{NO}_{\mathrm{x}}$ formation during high temperature processes include thermal $\mathrm{NO}_{\mathrm{x}}$, prompt $\mathrm{NO}_{\mathrm{x}}$, and fuel 
$\mathrm{NO}_{\mathrm{x}}^{[22],[23]}$. Thermal $\mathrm{NO}_{\mathrm{x}}$ forms by the combination of oxygen and nitrogen radicals that are released during combustion by the following overall reaction:

$$
\begin{gathered}
O_{2(g)} \leftrightarrow 2 O_{(g)} \\
O_{(g)}+2 N_{(g)} \leftrightarrow N O_{(g)}+N_{(g)} \\
N_{(g)}+O_{2(g)} \leftrightarrow N O_{(g)}+O_{(g)} \\
- \\
O_{2(g)}+N_{2(g)} \leftrightarrow 2 N O_{(g)} \\
N O_{(g)}+\frac{1}{2} O_{2(g)} \leftrightarrow N O_{2(g)}
\end{gathered}
$$

Prompt $\mathrm{NO}_{\mathrm{x}}$ formation occurs at the edges of the flame zone, where oxygen and hydroxide radicals increase the rate of formation of $\mathrm{NO}_{\mathrm{x}}{ }^{[22]}$. Fuel $\mathrm{NO}_{\mathrm{x}}$ forms from nitrogenous sources of fuel combusting and reacting with oxygen. These fuels include ammonia $\left(\mathrm{NH}_{3}\right)$ and nitrogen organically bound to hydrocarbons.

Nitrogen oxides in the atmosphere are involved in the formation of ozone in the troposphere and acid rain. In the presence of high energy light $(<398 \mathrm{~nm})$, nitrogen dioxide is broken down, which allows the formation of ozone:

$$
\begin{array}{r}
N O_{2(g)} \stackrel{h v}{\rightarrow} N O_{(g)}+O_{(g)} \\
O_{(g)}+O_{2(g)}+M_{(g)} \rightarrow O_{3(g)}+M_{(g)}
\end{array}
$$

where $\mathrm{M}$ is a molecule that accepts energy lost in the reaction as kinetic energy. The reaction continues as NO reacts with ozone to complete the cycle.

$$
N O_{(g)}+\mathrm{O}_{3(g)} \rightarrow \mathrm{NO}_{2(g)}+\mathrm{O}_{2(g)}
$$

In the presence of moisture in the atmosphere, nitrogen oxide can react with hydroxyl radicals to form nitric acid as shown in equation 9.

$$
\begin{aligned}
\mathrm{M}_{(g)}+\mathrm{H}_{2} \mathrm{O}_{(g)} & \rightarrow \mathrm{MH}_{(g)}+\mathrm{OH}_{(g)}^{-} \\
\mathrm{OH}_{(g)}^{-} & +\mathrm{NO}_{2(g)} \rightarrow \mathrm{HNO}_{3}(g)
\end{aligned}
$$


where $\mathrm{M}$ is a molecule that will accept a proton. The combination of these reactions results in ozone formation and acid rain, which forms when the nitric acid encounters water droplets and falls out of the atmosphere as rain. These cause health issues, acidification of water bodies, and damage to plants ${ }^{[24]}$.

\section{$\underline{3.1 .2 \mathrm{NO}_{\underline{x}} \text { Control Technologies }}$}

The two main techniques for control of $\mathrm{NO}_{\mathrm{x}}$ compounds include combustion controls and emission controls. Combustion controls involve modifying the conditions of the combustion reaction such as the temperature, airflow, fuel air chemistry, or the processing method. For plasma gasification, the temperature depends on the torch used, the power input, and the process gas used. To treat a wide variety of waste materials, the goal is to maximize the temperature to reduce the destruction time for waste materials. Modifying the temperature for $\mathrm{NO}_{\mathrm{x}}$ reduction in this system is therefore not a viable option. One benefit of plasma gasification is the ability to change the gas used by the torch. Air is composed of $78 \%$ nitrogen, so if the process gas is then switched to steam, argon, helium or another gas that does not contain nitrogen, then the only source of nitrogen would be from the waste to be treated.

The main technique for $\mathrm{NO}_{\mathrm{x}}$ emissions control in plasma gasification is to control the flue gas. Flue gas emission controls are considered secondary control technologies located downstream of the combustion zone. Some of these secondary controls require the gases to be treated to remain at high temperature for the chemicals reactions to favorably occur. Several of these secondary control technologies are discussed, along with the pros and cons of each process.

\subsubsection{Selective non-catalytic reduction (SCNR)}

Selective non-catalytic reduction (SCNR) is a high temperature reaction that involves injecting ammonia $\left(\mathrm{NH}_{3}\right)$ or urea $\left(\left(\mathrm{NH}_{2}\right)_{2} \mathrm{CO}\right)$ into the gas stream above the combustion zone to

reduce $\mathrm{NO}_{\mathrm{x}}$ to nitrogen gas $\left(\mathrm{N}_{2}\right)$ and water ${ }^{[22]}$. This process typically reduces the amount of $\mathrm{NO}_{\mathrm{x}}$ by 30 to $50 \%{ }^{[22]}$. If urea is used, the first reaction is urea breaking down into ammonium ion $\left(\mathrm{NH}_{4}{ }^{+}\right)$and carbamate ion $\left(\mathrm{NH}_{2} \mathrm{COO}^{-}\right)$and then into $\mathrm{NH}_{3}$ and $\mathrm{CO}_{2}$ by the following reactions:

$$
\mathrm{H}_{2} \mathrm{NCONH}_{2(\mathrm{aq})}+\mathrm{H}_{2} \mathrm{O}_{(\mathrm{g})} \stackrel{\text { heat }}{\longrightarrow} \mathrm{NH}_{2} \mathrm{COO}_{(\mathrm{g})}^{-}+\mathrm{NH}_{4(g)}^{+} \rightarrow 2 \mathrm{NH}_{3(g)}+\mathrm{CO}_{2(g)}
$$


Once the $\mathrm{NH}_{3}$ is free, it will react with $\mathrm{NO}$ and oxygen in the gas stream to form $\mathrm{N}_{2}$ and water by either of the following reactions depending on the temperature:

$$
\begin{array}{rlr}
2 \mathrm{NH}_{3(g)}+2 \mathrm{NO}_{(g)}+\mathrm{O}_{2(g)} & \rightarrow 2 \mathrm{~N}_{2(g)}+3 \mathrm{H}_{2} \mathrm{O}_{(g)} & 871-1038^{\circ} \mathrm{C} \\
2 \mathrm{NH}_{3(g)}+2 \mathrm{NO}_{(g)}+\mathrm{O}_{2(g)}+\mathrm{H}_{2(g)} & \rightarrow 2 \mathrm{~N}_{2(g)}+4 \mathrm{H}_{2} \mathrm{O}_{(g)} & 704-1038^{\circ} \mathrm{C}
\end{array}
$$

The presence of hydrogen gas allows the reaction to occur at lower temperatures. These reactions do not need a catalyst to occur yet do require enough residence time to react fully. Above $1038^{\circ} \mathrm{C}$, the upper limit of the temperature for the reactions shown in equations 12 and 13 , the ammonia will break down into more NO, which is what the process is attempting to remove:

$$
4 \mathrm{NH}_{3(g)}+5 \mathrm{O}_{2(g)} \rightarrow 4 \mathrm{NO}_{(g)}+6 \mathrm{H}_{2} \mathrm{O}_{(g)} \quad>1038^{\circ} \mathrm{C}
$$

Building a system that meets these requirements of $\mathrm{NH}_{3}$ stored nearby and minimum residence time at temperatures greater than $704^{\circ} \mathrm{C}$ adds cost and would likely require the system to be stationary at a set facility. The goal of this project is to make a low cost system that can be mobile to reach parts of the world that do not have effective waste management systems. The need for $\mathrm{NH}_{3}$ storage also complicates this design as many parts of the world where this process could improve conditions may not have access to high purity $\mathrm{NH}_{3}$.

\subsubsection{Selective catalytic reduction (SCR)}

Selective catalytic reduction (SCR) is similar to SCNR with a catalyst bed that can lower the effective temperature of the reaction. The SCR system involves injection of a reducing agent, typically ammonia, upstream of a catalyst bed that the flue gas contacts. The dominant reactions occurring in SCR systems include that of equation 12 at lower temperatures, and the following:

$$
6 \mathrm{NO}_{(g)}+4 \mathrm{NH}_{3(g)} \rightarrow 5 \mathrm{~N}_{2(g)}+6 \mathrm{H}_{2} \mathrm{O}_{(g)}
$$

The temperature range of the reaction depends on the catalyst material used. Precious metal catalysts will reduce the temperature of reaction to a range of $175^{\circ}-290^{\circ} \mathrm{C}^{[20]}$. For large-scale treatment, this can become expensive; however the operating costs can be far lower with an 
operating temperature at this range. A more common catalyst is vanadium pentoxide on titanium dioxide, which reacts in the range of $260^{\circ}-425^{\circ} \mathrm{C}^{[22]}$. Zeolites can also be used in the range of $454^{\circ}-593{ }^{\circ} \mathrm{C}^{[22]}$.

There are common issues with the catalytic bed in SCR making the process less desirable under certain situations. For example, if the flue gas contains sulfur, the catalyst will increase the rate of oxidation of $\mathrm{SO}_{2}$ to $\mathrm{SO}_{3}$ resulting in sulfuric acid mist emissions. This can be reduced by using tungsten trioxide or molybdenum trioxide catalysts at the downstream end of the catalyst bed. The catalyst beds can also be overwhelmed with particulates, greatly reducing their effectiveness. To counteract this, the SCR must simply be placed downstream of particulate control systems. Overall, SCR systems are capable of between 70\%-90\% reduction of $\mathrm{NO}_{\mathrm{x}}$.

\subsubsection{Catalytic absorption}

Catalytic absorption is an absorption process that is modified with a catalyst. One commercial technology uses a potassium carbonate coating on the catalyst to remove both $\mathrm{CO}$ and $\mathrm{NO}_{\mathrm{x}}$. The $\mathrm{NO}$ and $\mathrm{CO}$ are first oxidized to $\mathrm{NO}_{2}$ and $\mathrm{CO}_{2}$ and are then absorbed on the catalyst by the following reaction:

$$
2 \mathrm{NO}_{2(g)}+\mathrm{K}_{2} \mathrm{CO}_{3(s)} \rightarrow \mathrm{CO}_{2(g)}+\mathrm{KNO}_{2(s)}+\mathrm{KNO}_{3(s)}
$$

Once the carbonate coating is depleted, it must be regenerated using a dilute stream of hydrogen and carbon dioxide in the absence of oxygen ${ }^{[22]}$ :

$$
\mathrm{KNO}_{2(s)}+\mathrm{KNO}_{3(s)}+4 \mathrm{H}_{2(g)}+\mathrm{CO}_{2(g)} \rightarrow \mathrm{K}_{2} \mathrm{CO}_{3(s)}+4 \mathrm{H}_{2} \mathrm{O}_{(g)}+\mathrm{N}_{2(g)}
$$

The oxidation/absorption cycle lasts between 9 and 15 minutes before requiring regeneration, and the regeneration takes 3 to 5 minutes. With such a short duration of useful absorption, many individual sections of catalyst bed are required, with a portion of the beds being regenerated all the time. The regenerated portion must be isolated from the exhaust gases to prevent the presence of oxygen. This process occurs effectively between $150{ }^{\circ} \mathrm{C}$ and $370^{\circ} \mathrm{C}$, and does not require ammonia storage (required for both SCNR and SCR). This process is used in conjunction with other emission control technologies to increase its effectiveness. 
There are several issues with catalytic absorption being used in this small scale system. The constant regeneration of catalyst beds makes this process slightly more complicated in design. Sealing each of the beds from the exhaust gases and introducing a mixture of hydrogen and carbon dioxide would involve extensive ducting and valves to be installed and maintained. The hydrogen and carbon dioxide mixture would require a storage tank to be in close proximity. Alternatively, another system of gas separation from the final exhaust to extract these gases without oxygen could be used. This system should be automated for the lowest maintenance.

\subsubsection{Low temperature oxidation with absorption}

Low temperature oxidation with absorption involves a reaction with ozone to form a highly soluble byproduct, $\mathrm{N}_{2} \mathrm{O}_{5}$, which can be absorbed in a wet scrubber. The reaction occurs in the following sequence ${ }^{[22]}$ :

$$
\begin{gathered}
\mathrm{NO}_{(g)}+\mathrm{O}_{3(g)} \rightarrow \mathrm{NO}_{2(g)}+\mathrm{O}_{2(g)} \\
2 \mathrm{NO}_{2(g)}+\mathrm{O}_{3(g)} \rightarrow \mathrm{N}_{2} \mathrm{O}_{5(g)}+\mathrm{O}_{2(g)} \\
\mathrm{N}_{2} \mathrm{O}_{5(g)}+\mathrm{H}_{2} \mathrm{O}_{(l)} \rightarrow 2 \mathrm{H}_{(a q)}^{+}+2 \mathrm{NO}_{3(a q)}^{-}
\end{gathered}
$$

The oxidation of $\mathrm{NO}_{2}$ and $\mathrm{NO}$ are faster than the competing reactions of $\mathrm{CO}$ and $\mathrm{SO}_{2}$ and will not compete with consumption of ozone. This process is highly efficient when used with the

exhaust from combustion products and can reach up to $99 \% \mathrm{NO}_{\mathrm{x}}$ removal ${ }^{[22]}$. The reactions with ozone occur at temperatures of approximately $150{ }^{\circ} \mathrm{C}$ and the destruction of ozone occurs at 260 ${ }^{\circ} \mathrm{C}^{[22]}$. These temperatures are reasonable for a $\mathrm{NO}_{\mathrm{x}}$ control process at a fixed facility wherein the byproduct of nitric acid $\left(\mathrm{HNO}_{3}\right)$ could be appropriately managed. This process also requires the on-site storage of oxygen for production of ozone adding substantial cost and size of the facility.

\subsubsection{Corona-induced plasma}

Corona-induced plasma or corona discharge is non-thermal plasma generated by a high electric field surrounding a conductor and produces a stream of ionized gas. The radicals produced in the plasma can oxidize $\mathrm{NO}$ to $\mathrm{NO}_{2}$ and $\mathrm{N}_{2} \mathrm{O}_{5}$ as described in equations 18,19 and 
20. The byproduct of these reactions is nitric acid, which would need to be dealt with in the scrubber reservoir.

\subsubsection{Reburning}

Reburning consists of burning the exhaust gases at a lower temperature in fuel rich conditions and allows for combustion control techniques to be used to reduce the amount of $\mathrm{NO}_{\mathrm{x}}$ produced $^{[23]}$. Reburning requires the addition of fuel and a combustion zone beyond the plasma chamber itself. This defeats the purpose of using a plasma system with no combustion zone, as it introduces all the exhaust components of combustion after the plasma chamber.

For the small scale system in this project, a catalytic converter paired with a packed bed scrubber was selected for $\mathrm{NO}_{\mathrm{x}}$ control. These two control methods do not require storage of ammonia, oxygen, hydrogen or carbon dioxide. A catalytic converter is a low cost option for $\mathrm{NO}_{\mathrm{x}}$ reduction compared to the other catalyst methods described. With further testing, additional modifications or systems may be added for better $\mathrm{NO}_{\mathrm{x}}$ control.

\subsection{Ozone}

Ozone formation occurs within the plasma by the breakdown and re-combination of oxygen atoms. Ozone has a decreasing half-life at increasing temperatures. The half-life of ozone at $20^{\circ} \mathrm{C}$ is 3 days and as the temperature increases to $250^{\circ} \mathrm{C}$, the half-life decreases to 1.5 seconds ${ }^{[26]}$. Given the high temperatures expected in the plasma chamber, ozone forming in the plasma reaction chamber will only be present in the plasma for a very short time as it will break down into $\mathrm{O}_{2}$ and $\mathrm{O}^{2-}$. If there are any ozone molecules that do end up lasting long enough to interact with other molecules, those ozone molecules would be helpful in breaking down other molecules. Further in the exhaust stream, where the temperature is low enough, ozone may form yet. However, considering its high reactivity ozone will likely react with other exhaust byproducts before it can escape the system. Ozone has been used as a method for breaking down

organic molecules in water treatment and in treatment of VOCs in exhaust processes ${ }^{[25]}$. Within the plasma chamber, ozone will have a beneficial effect. 


\subsection{Carbon Monoxide}

Carbon monoxide forms through incomplete combustion of carbonaceous fuels. The basic reactions of carbon in combustion are:

$$
\begin{aligned}
2 C_{(g)}+O_{2(g)} & \rightarrow 2 \mathrm{CO}_{(g)} \\
2 \mathrm{CO}_{(g)}+O_{2(g)} & \rightarrow 2 \mathrm{CO}_{2(g)}
\end{aligned}
$$

The first reaction occurs at a much faster rate than the second. If the combustion reaction is oxygen limited, then there will be far more CO produced. The air to fuel ratio is generally increased to the correct stoichiometric ratio or with excess air to push the reaction toward $\mathrm{CO}_{2}$ production.

The catalytic converter is used in combination with internal combustion engines to control both $\mathrm{CO}$ and $\mathrm{NO}_{\mathrm{x}}$ emissions. During combustion in air, the nitrogen present is oxidized to form $\mathrm{NO}_{\mathrm{x}}$. Catalytic converters in automobiles typically use platinum, palladium and/or rhodium as the catalyst material. Platinum is used for both oxidation and reduction, while palladium is used for oxidation, and rhodium for reduction ${ }^{[27]}$. The following reactions occur in the catalytic converter to reduce NO and oxidize carbon ${ }^{[28]}$ :

$$
\begin{aligned}
\mathrm{NO}_{(g)}+\mathrm{C}_{n} \mathrm{H}_{m(g)}(\text { or } \mathrm{CO}) & \rightarrow \mathrm{N}_{2(g)}+\mathrm{CO}_{2}(g) \\
\mathrm{C}_{n} \mathrm{H}_{m(g)}+\mathrm{O}_{2(g)} & \rightarrow \mathrm{CO}_{2}(g) \\
2 \mathrm{CO}_{(g)}+\mathrm{O}_{2(g)} & \rightarrow 2 \mathrm{CO}_{2(g)} \\
\mathrm{CH}_{2} \mathrm{O}_{(g)}+\mathrm{O}_{2(g)} & \rightarrow \mathrm{CO}_{2(g)}+\mathrm{H}_{2} \mathrm{O}_{(g)}
\end{aligned}
$$

where $\mathrm{n}$ and $\mathrm{m}$ are the number of carbon and hydrogen atoms in various hydrocarbon fuels. When running an internal combustion engine with a fuel rich air/fuel ratio, there is not enough oxygen to fully oxidize the carbon into $\mathrm{CO}_{2}$. When running an internal combustion engine with a lean air/fuel ratio, there is not enough unburnt carbon to reduce $\mathrm{NOx}$ to $\mathrm{N}_{2}$. It is therefore important to run an internal combustion engine at the right air/fuel ratio to maximize the conversion of $\mathrm{CO}$ to $\mathrm{CO}_{2}$, $\mathrm{NO}$ to $\mathrm{N}_{2}$ and unburned hydrocarbons to $\mathrm{CO}_{2}$ and $\mathrm{H}_{2} \mathrm{O}$ within the catalytic converter. 
In industrial combustion applications, the main forms of $\mathrm{CO}$ controls are allowing sufficient residence time, air/fuel ratio control, air injection to secondary burners, and keeping the temperature up to allow for maximum oxidation of carbon ${ }^{[29]}$. Afterburners or chambers kept above $760{ }^{\circ} \mathrm{C}$ are used to increase residence time and maintain the high temperature environment for the reactions to continue ${ }^{[29]}$. Combustion zones can be kept between $870{ }^{\circ} \mathrm{C}$ and $980{ }^{\circ} \mathrm{C}$ to achieve removal efficiencies of over $95 \%{ }^{[29]}$. Thermal incinerators and catalytic incinerators make up the majority of $\mathrm{CO}$ control techniques in industrial processes. These incinerators are chambers downstream of the combustion chamber kept at temperatures that will allow complete oxidation of carbon with or without catalysts and often have air injection to provide oxygen ${ }^{\text {[30] }}$.

A catalytic converter was selected for carbon monoxide control. The catalytic converter will both oxidize carbon monoxide and reduce NOx, making it a dual purpose system. The length of piping from the gasification chamber to the downstream end of the catalytic converter will be insulated to keep the temperature high for additional residence time. These two methods should control the majority of the carbon monoxide produced. Further testing will determine whether additional controls are necessary.

\subsection{Sulfur Dioxide}

\subsubsection{Sulfur Dioxide Formation}

Sulfur is the tenth most common element by mass and can be found in many materials including rubber, paper, amino acids, insecticides and fungicides, and hydrocarbon fuel sources. When these materials are broken down by combustion, sulfur dioxide is formed:

$$
S_{(s)}+O_{2(g)} \stackrel{\text { heat }}{\longrightarrow} S O_{2}(g)
$$

Sulfur dioxide in the atmosphere leads to acid rain and acidification of water bodies, various human health issues, and damage to foliage. Once in the atmosphere, sulfur dioxide can react with water and oxygen to form sulfuric acid, which can then be deposited on the earth's surface with rain by the following equations:

$$
\begin{gathered}
2 \mathrm{SO}_{2(g)}+\mathrm{O}_{2(g)} \rightarrow 2 \mathrm{SO}_{3(g)} \\
\mathrm{SO}_{3(g)}+\mathrm{H}_{2} \mathrm{O}_{(l)} \rightarrow \mathrm{H}_{2} \mathrm{SO}_{4(a q)}
\end{gathered}
$$


The combination of sulfuric and nitric acids in acid rain have caused some lakes in the Adirondack Mountains of New York State, in Canada, and in Scandinavia to reach low enough $\mathrm{pH}$ values that all the fish have died ${ }^{[24]}$. The largest source of sulfur emissions is from the burning of coal ${ }^{[22]}$. The majority of $\mathrm{SO}_{2}$ emissions causing acid rain in the United States have been reduced due to strict controls on the exhaust of the power generating industry with the Acid Rain $\mathrm{SO}_{2}$ Reduction Program established under Title IV of the Clean Air Act Amendments of $1990^{[31]}$.

\subsubsection{Sulfur Dioxide Control Technologies}

\subsubsection{Calcium based wet reactions}

The wet limestone method of flue gas desulfurization (FGD) uses wet limestone dissolved in water to react with dissolved $\mathrm{SO}_{2}{ }^{[30]}$. This process requires a spray tower where the spray of water is run toward the incoming exhaust gas to allow $\mathrm{SO}_{2}$ to partition into the water. The majority of the chemistry occurs in the reservoir where calcium sulfite and calcium sulfate are produced. This process typically uses slurry concentrations of $10 \%$ to $15 \%$ solids by weight with a retention time of 12 to 14 hours $^{[30]}$.

Wet lime FGD commonly uses a countercurrent flow vertical spray tower with a hydrated lime slurry spray ${ }^{[32]}$. The process is similar to wet limestone FGD, using hydrated lime instead of limestone and is often enhanced with magnesium to increase removal efficiency. The magnesium-enhanced lime (MEL) process uses 5\% to $8 \%$ magnesium oxide or dolomitic lime with approximately $20 \%$ magnesium oxide ${ }^{[30]}$. With increased removal efficiency, the MEL process allows for smaller absorbers than that of the wet limestone FGD. The MEL process also has the potential to produce a higher quantity of gypsum $\left(\mathrm{CaSO}_{4} * 2 \mathrm{H}_{2} \mathrm{O}\right)$ in the reservoir.

Both wet limestone FGD and wet lime FGD processes have the potential for gypsum scale to form in the absorbers and process parameters must be followed to reduce operating and maintenance costs. By blowing air through the reservoir, gypsum can be produced in the reservoir to avoid formation in the absorber. Alternatively, emulsified sodium thiosulfate $\left(\mathrm{Na}_{2} \mathrm{~S}_{2} \mathrm{O}_{3}\right)$ can be added to prevent gypsum formation in the absorber ${ }^{[22],[30]}$. Sodium thiosulfate is added for systems with higher sulfur content. 


\subsubsection{Calcium based dry reactions}

Lime dry spraying FGD involves spraying a hydrated lime slurry into the hot flue gas stream within a spray drying chamber ${ }^{[30]}$. The flue gas must be hot, as the reaction of $\mathrm{CaO}$ and $\mathrm{SO}_{2}$ occurs at approximately $1000{ }^{\circ} \mathrm{C}^{[30]}$. This is considered a dry process as the spray quickly evaporates to leave behind particulates that have sorbed the $\mathrm{SO}_{2}$. These particulates must then be removed during a subsequent process.

Duct sorbent injection is similar to lime dry spraying, without the spray drying chamber. The hydrated lime slurry is injected directly into the flue gas duct with water being injected further downstream ${ }^{[30]}$. These particulates also need to be removed in a subsequent process.

Furnace sorbent injection involves injecting calcium hydroxide or calcium carbonate just above or downstream of the combustion chamber to form highly porous particulate with high surface area. This greatly increases the ability to sorb $\mathrm{SO}_{2}$. This process also forms particulate matter to be removed.

Circulating fluidized beds use a bed of calcium hydroxide that is kept continuously moving with the flue gas circulating throughout the bed material. This process allows for high contact time with the calcium hydroxide. Circulating fluidized beds introduce a high concentration of particulates that need to be removed downstream.

The particulates formed in the dry processes are easier to remove than those of the wet processes. This is often the reason to use dry systems over wet systems. Operating and maintenance costs tend to be the determining factor for which of these systems to use, along with preferences of handling the reaction products. Some facilities may choose to handle a slurry or aqueous solution over dry powder or vice versa.

\subsubsection{Claus Process}

The Claus process uses hydrogen sulfide $\left(\mathrm{H}_{2} \mathrm{~S}\right)$ to react with the $\mathrm{SO}_{2}$ produced to form elemental sulfur ${ }^{[22]}$. This process assumes there will be $\mathrm{H}_{2} \mathrm{~S}$ present as the main gaseous form of sulfur and requires a portion of that $\mathrm{H}_{2} \mathrm{~S}$ to be burned to form $\mathrm{SO}_{2}$. The mixture is then passed over a catalyst to produce elemental sulfur by the following reaction:

$$
2 \mathrm{H}_{2} \mathrm{~S}_{(g)}+\mathrm{SO}_{2(g)} \rightarrow 2 \mathrm{H}_{2} \mathrm{O}_{(g)}+3 \mathrm{~S}_{(s)}
$$


This process requires that there be two moles of $\mathrm{H}_{2} \mathrm{~S}$ for every mole of $\mathrm{SO}_{2}$ which can be a challenge to achieve in a high velocity gas flow. The Claus process can remove 93 to $97 \%$ of the sulfur, with the remaining sulfur likely to be small amounts of unreacted $\mathrm{H}_{2} \mathrm{~S}, \mathrm{SO}_{2}$ and other sulfur compounds such as carbon disulfide $\left(\mathrm{CS}_{2}\right)$, carbonyl sulfide (COS), and sulfur vapor $\left(\mathrm{S}_{8}\right)^{[22]}$. These trace components in the exhaust gas would need an additional process to remove them from the gas stream.

\subsubsection{Calcium based reaction chemistry}

The chemistry of calcium based wet and dry FGD processes are described by similar stoichiometric reactions. Limestone $\left(\mathrm{CaCO}_{3}\right)$ is used to produce lime $\left(\mathrm{Ca}(\mathrm{OH})_{2}\right)$ by the following equations:

$$
\begin{gathered}
\mathrm{CaCO}_{3} \stackrel{\text { heat }}{\longrightarrow} \mathrm{CaO}_{(s)}+\mathrm{CO}_{2}(\mathrm{~g}) \\
\mathrm{CaO}_{(\mathrm{s})}+\mathrm{H}_{2} \mathrm{O}_{(\mathrm{l})} \rightarrow \mathrm{Ca}(\mathrm{OH})_{2(a q)}
\end{gathered}
$$

For the wet limestone $\mathrm{FSD}$, the $\mathrm{SO}_{2}$ reacts to form $\mathrm{CaSO}_{4}$ in the reservoir by the following equations:

$$
\begin{gathered}
\mathrm{CaCO}_{3(s)}+\mathrm{SO}_{2(a q)}+\frac{1}{2} \mathrm{H}_{2} \mathrm{O}_{(l)} \rightarrow \mathrm{CaSO}_{3} * \frac{1}{2} \mathrm{H}_{2} \mathrm{O}_{(s)}+\mathrm{CO}_{2}(g) \\
2 \mathrm{CaSO}_{3} * \frac{1}{2} \mathrm{H}_{2} \mathrm{O}_{(s)}+3 \mathrm{H}_{2} \mathrm{O}_{(l)}+\mathrm{O}_{2(g)} \rightarrow 2 \mathrm{CaSO}_{4} * 2 \mathrm{H}_{2} \mathrm{O}_{(\mathrm{s})}
\end{gathered}
$$

The wet lime, lime dry spraying, and hydrated lime FGD processes form the same products by the following reactions:

$$
\begin{gathered}
\mathrm{Ca}(\mathrm{OH})_{2(a q)}+\mathrm{SO}_{2(g)} \rightarrow \mathrm{CaSO}_{3} * \frac{1}{2} \mathrm{H}_{2} \mathrm{O}_{(\mathrm{s})}+\frac{1}{2} \mathrm{H}_{2} \mathrm{O}_{(l)} \\
\mathrm{Ca}(\mathrm{OH})_{2(a q)}+\mathrm{SO}_{2(a q)}+\frac{1}{2} \mathrm{O}_{2(a q)}+\mathrm{H}_{2} \mathrm{O}_{(l)} \rightarrow \mathrm{CaSO}_{4} * 2 \mathrm{H}_{2} \mathrm{O}_{(\mathrm{s})}
\end{gathered}
$$

Calcium sulfite forms needle-like crystals that are hard to dewater, has no market value, and is typically sent to landfills. Pure calcium sulfate is gypsum, which does have market value and can be made into commercial products such as plaster, drywall, and as an additive in cement, glass, stucco and other chemicals. Dry calcium based FGD systems typically contain approximately $75 \%$ calcium sulfite and $25 \%$ calcium sulfate. This mixture can be sent to a landfill and will cause the leachate to have a high $\mathrm{pH}$. 


\subsubsection{Sodium Based Reactions}

\subsubsection{Wet sodium based scrubbers}

Wet sodium scrubbers will use either a sodium carbonate $\left(\mathrm{Na}_{2} \mathrm{CO}_{3}\right)$ or sodium hydroxide $(\mathrm{NaOH})$ solution $^{[22]}$. Sodium carbonate is dissolved into solution to form carbonate and bicarbonate ions by the following equations:

$$
\begin{gathered}
\mathrm{NaCO}_{3(s)}+\mathrm{H}_{2} \mathrm{O}_{(l)} \rightarrow \mathrm{Na}_{(a q)}^{+}+\mathrm{CO}_{3}^{2-}(a q) \\
\mathrm{CO}_{3}^{2-}(a q) \\
+\mathrm{H}_{2} \mathrm{O}_{(l)} \mathrm{O}_{(l)}
\end{gathered}
$$

The alternative sodium technique, using sodium hydroxide, skips the dissolution step required on using sodium carbonate as $\mathrm{NaOH}$ is sold in solution. Either of these solutions would then be sprayed through a scrubber where the $\mathrm{SO}_{2(\mathrm{~g})}$ would be absorbed into solution. The sprayer solution's alkalinity prevents the build-up of sulfurous acid by pushing the reaction to favor sulfite ions in solution:

$$
\begin{aligned}
& \mathrm{SO}_{2}(g)+\mathrm{H}_{2} \mathrm{O}_{(l)} \leftrightarrow \mathrm{H}_{2} \mathrm{SO}_{3}(a q) \\
& \mathrm{H}_{2} \mathrm{SO}_{3(a q)} \leftrightarrow H_{(a q)}^{+}+\mathrm{HSO}_{3}^{-}(a q) \\
& H_{(a q)}^{+}+\mathrm{HSO}_{3(a q)}^{-} \leftrightarrow S \mathrm{O}_{3(a q)}^{2-}+2 H_{(a q)}^{+}
\end{aligned}
$$

The overall reactions in the scrubber solution would then be:

$$
\begin{aligned}
2 \mathrm{Na}_{(a q)}^{+}+2 \mathrm{OH}_{(a q)}^{-}+\mathrm{SO}_{2(a q)} & \rightarrow \mathrm{Na}_{2} \mathrm{SO}_{3(a q)}+\mathrm{H}_{2} \mathrm{O}_{(l)} \\
2 \mathrm{Na}_{(a q)}^{+}+2 \mathrm{OH}_{(a q)}^{-}+\mathrm{SO}_{2(a q)}+\frac{1}{2} \mathrm{O}_{2(a q)} & \rightarrow \mathrm{Na}_{2} \mathrm{SO}_{4(a q)}+\mathrm{H}_{2} \mathrm{O}_{(l)} \\
2 \mathrm{Na}_{(a q)}^{+}+\mathrm{OH}_{(a q)}^{-}+\mathrm{SO}_{2(a q)} & \rightarrow \mathrm{Na}_{2} \mathrm{HSO}_{3(a q)}
\end{aligned}
$$

where the products are a mixture of sodium sulfite, sodium sulfate and sodium bisulfite. The dominant species would be sodium bisulfite if the $\mathrm{pH}$ of the solution is low.

\subsubsection{Dry sodium based systems}

Dry sodium systems use sodium bicarbonate $\left(\mathrm{NaHCO}_{3}\right)$ or sodium sesquicarbonate $\left(\mathrm{Na}_{3} \mathrm{H}\left(\mathrm{CO}_{3}\right)_{2}\right)$ exposed to heat $\left(150{ }^{\circ} \mathrm{C}-315^{\circ} \mathrm{C}\right)$ to produce particulates with high surface area for maximum sorption ${ }^{[22]}$. Sodium sesquicarbonate is made up of both sodium carbonate and 
sodium bicarbonate. With heat, the sodium bicarbonate is broken down into sodium carbonate, which can then react with the $\mathrm{SO}_{2}$ by the following reactions:

$$
\begin{aligned}
& 2 \mathrm{NaHCO}_{3(s)} \stackrel{\text { heat }}{\longrightarrow} \mathrm{Na}_{2} \mathrm{CO}_{3(s)}+\mathrm{CO}_{2(g)}+\mathrm{H}_{2} \mathrm{O}_{(l)} \\
& \mathrm{Na}_{2} \mathrm{CO}_{3(s)}+\mathrm{SO}_{2(g)} \rightarrow \mathrm{Na}_{2} \mathrm{SO}_{3(s)}+\mathrm{CO}_{2(g)}
\end{aligned}
$$

The sodium sulfite product can then be removed from the system. Sodium sulfate and sodium sulfite are both of commercial value and could be sold where there is demand.

A packed bed scrubber with a $\mathrm{NaOH}$ solution was selected in this system for $\mathrm{SO}_{2}$ control. Sodium hydroxide solution was available in the Contaminant Transport and Chemistry Lab, so it was selected for the scrubber solution.

\subsection{Particulate Matter}

\subsubsection{Particulate Matter Formation}

Particulate matter (PM) consists of solid particles and liquid droplets that are suspended in the air. These particles can be liquid droplets, solid particles, or a combination such as a particle of black carbon from diesel exhaust coated in a thin layer of water that has condensed onto it. Particles are categorized by size with particles under $10 \mu \mathrm{m}$ in diameter called PM10 and particles under $2.5 \mu \mathrm{m}$ in diameter called PM2.5. The PM10 particles are small enough to can get into the lungs, with larger particles depositing on the mouth, throat and upper regions of the

lungs, and smaller particles (mainly PM2.5) reaching deep into the lungs ${ }^{[28]}$. A portion of PM2.5 can diffuse directly into the bloodstream through the alveoli due to their small size ${ }^{[28]}$.

Particles can form either physically or chemically from primary sources or as secondary pollutants ${ }^{[28]}$. Primary particles include soot and combustion related particles, and particles that are physically released from mechanical processes such as grinding, or machining. Primary gaseous pollutants can react to form secondary particulate pollutants. This occurs by reactions of $\mathrm{SO}_{2}, \mathrm{NO}_{\mathrm{x}}, \mathrm{NH}_{3}$, and VOCs to form other compounds which are considered particulates ${ }^{[28]}$. Sulfur dioxide will form sulfuric acid $\left(\mathrm{H}_{2} \mathrm{SO}_{4}\right)$ and the associated ions $\left(\mathrm{HSO}_{4}{ }^{-}\right.$and $\left.\mathrm{SO}_{4}{ }^{2-}\right)$. Nitrogen oxides will form nitric acid $\left(\mathrm{HNO}_{3}\right.$ and $\left.\mathrm{NO}_{3}{ }^{-}\right)$, and ammonia will form ammonium ions $\left(\mathrm{NH}_{4}{ }^{+}\right)$. Ammonium will combine with both sulfuric and nitric acid ions to form ammonium nitrate $\left(\mathrm{NH}_{4} \mathrm{NO}_{3}\right)$ and ammonium sulfate $\left(\left(\mathrm{NH}_{4}\right)_{2} \mathrm{SO}_{4}\right)$, which are both considered particulates. The ions that form from these reactions can also combine with moisture in the air to form droplets. 
Volatile organic compounds will oxidize into less volatile compounds which can then condense and form secondary aerosols as well.

Particles larger than $10 \mu \mathrm{m}$ do not travel far and tend to settle out of suspension in the air. The smaller the particle, the further it can travel in the atmosphere. The gravitational settling velocity of a particle of $10 \mu \mathrm{m}$ diameter is approximately $1 \mathrm{~cm} / \mathrm{sec}$, depending on the density of the particle; the density, pressure and temperature of the air; and the motion of the air ${ }^{[32]}$. The gravitational settling velocity of a particle of $2.5 \mu \mathrm{m}$ diameter would be approximately 0.03 $\mathrm{cm} / \mathrm{sec}$ and that of $0.01 \mu \mathrm{m}$ diameter would be approximately $0.000015 \mathrm{~cm} / \mathrm{sec}^{[32]}$. These low settling velocities allow these small particles to remain suspended and travel long distances. With the circulation of the atmosphere, it takes airborne pollutants roughly two days to reach the top of the troposphere and approximately two months to reach the equator or the North Pole from the United States. In approximately two years, pollutants will reach the other hemisphere and, after roughly twenty years, pollutants will enter the stratosphere.

\subsubsection{Particulate Matter Control Technologies}

Mechanisms for particulate control involve three basic principles of impaction, interception and diffusion. Within a flowing gas stream, impaction is when the particles are large enough that their momentum allows them to leave their streamline and come forcibly into contact with a target (Figure 2) ${ }^{[22]}$. The smaller the radius of curvature of the streamline, the more likely a particle is to be carried out of it, so smaller targets are more likely to be impacted. Interception is when a particle is moving along its path of trajectory and that path curves around a target close enough that the particle comes in contact with the target (Figure 2). Interception occurs with particles between roughly $0.1 \mu \mathrm{m}$ to $1 \mu \mathrm{m}$ and only intercept when in a streamline sufficiently close to the target. Interception is therefore a weak mechanism for removal. Diffusion is when smaller submicron particles experience Brownian motion and randomly come into contact with the target (Figure 2). Diffusion occurs over short distances and long periods of time. The target in each of these cases can be the fibers in a filter screen, the side walls of a cyclone, water droplets in the spray of a wet scrubber, or some other physical object. 


\subsubsection{Cyclones}

A cyclone particle separator creates a vortex to use the principle of impaction to remove larger particles from the gas stream. The larger the particle, the more momentum the particle will have, which will cause the particle to come in contact with the side wall of the cyclone and fall down into a collection container. The particle trajectory varies directly with particle size (Figure 3$)^{[33]}$. The time a particle spends in the cyclone also increases the probability of a particle making contact with the side wall and falling out of suspension. Gases exit through a tube at the top of the cyclone where the velocity is lowest. This tube extends down into the cyclone to reduce the cross sectional area of the upper portion of the cyclone where the gas enters. This reduced cross sectional area helps to keep the velocity of the incoming gas high.
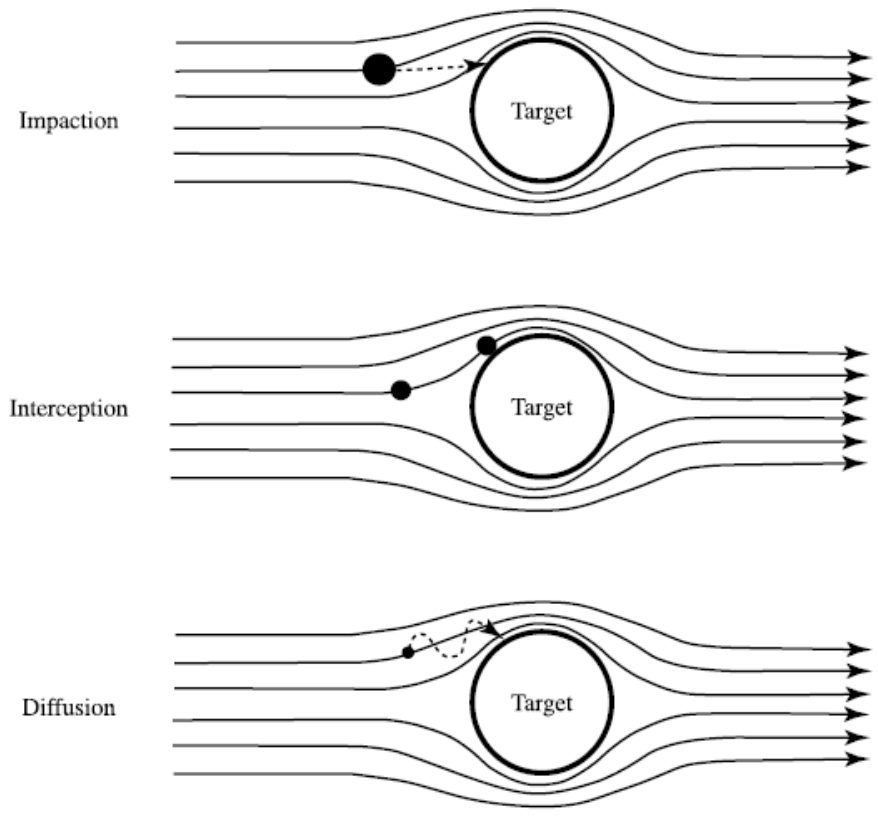

Figure 2: Particulate control mechanisms in a flowing gas stream $^{[20]}$. 

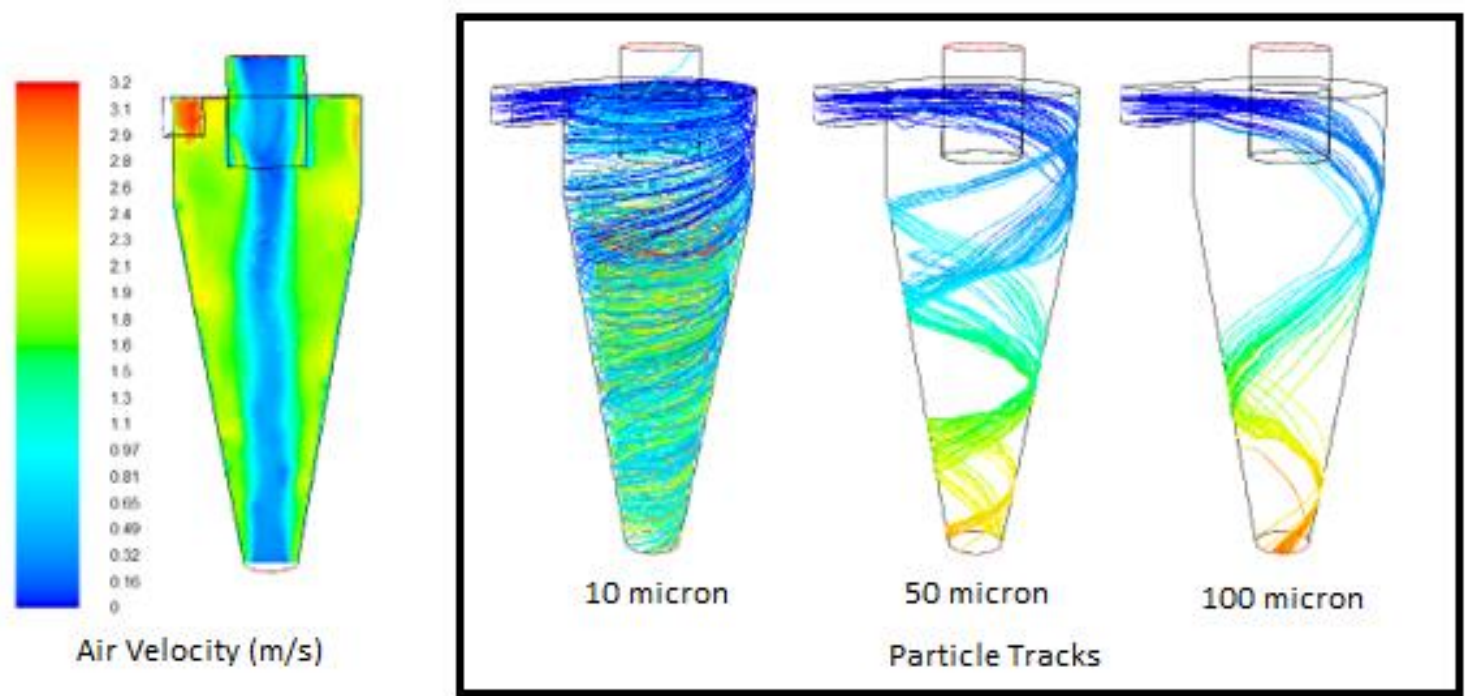

Figure 3: Particle trajectories in a cyclone particle separator of 10, 50 and 100 micron particles ${ }^{[33]}$.

Cyclones are only effective in capturing larger particles with enough momentum for impaction. Cyclones are low cost systems with minimal maintenance and simple designs, which makes them popular. There are no moving parts in a cyclone allowing them to last a long time. The main breakdown of cyclones is from abrasion of the particles along the inner wall. Cyclones often have a lining material such as a castable refractory or rubber to reduce abrasion. Cyclones can also handle high temperature gases depending on the material of construction. Overall, cyclones are a relatively inexpensive particle removal system for particles greater than $10 \mu \mathrm{m}$. Cyclones are often used to reduce the load on subsequent processes.

\subsubsection{Filtration}

Filtration of gas streams is done by placing some sort of filter media in the gas stream to provide a physical target for the particles to come into contact. The filter media can be a fabric material, porous ceramic, paper, activated carbon or any number of other materials. The mechanisms of particulate removal in filtration include impaction, interception and diffusion. Initially a clean filter will have impaction as the main mechanism. As more particles are embedded in the filter material, the path becomes more tortuous with smaller passages and particles are more likely to be removed by interception and diffusion ${ }^{[22]}$. Accordingly, filtration tends to do well with removing submicron particles. 
As mentioned above, the filtration media builds up a layer of particles over time. To prevent excess back pressure and to ensure continued removal of particles, filtration systems require physical replacement, physical removal, or back washing of the filter media. For example, applications employing bag filters often have a shaker that physically vibrates the bags to remove caked on dust. Bag systems can also deflate the bags and inflate to remove caked dust layers. The air flow can also be reversed or air pulsed at high pressure from the downstream side to push particles back off the filters.

Filter material properties are the most important design component to consider for the filtration of particulates. The properties of filter media to be considered include chemical resistance, maximum operating temperature, abrasion resistance and physical properties of the filter material such as weight, strength and weave ${ }^{[22]}$. The temperature of the gas flow is a major factor in material selection, with cost limiting how high of temperature to allow. There are plenty of options for low temperature filter material, with fewer low cost options as the operating temperature increases. As the temperature exceeds approximately $260{ }^{\circ} \mathrm{C}$, there are fewer options available ${ }^{[22]}$. High temperature filters include mainly ceramic fabrics or solid ceramic filters in the shape of cylinders (often called candles) ${ }^{[22]}$. Ceramic fiber filters and solid ceramic filters often have costs that are prohibitive to many designs ${ }^{[22]}$. It is often cheaper to reduce the temperature of the gas stream to be filtered than to select high temperature materials for the filtration media.

The chemistry of the gas stream will also affect the filtration material selection process. Commonly available filter materials have degrees of abrasion resistance, acid resistance, and base resistance. Choosing a material without the proper chemical or physical resistance will lead to failure, wasting both time and money. Filters tend to be less expensive than other consumables. Specifically, the operation and maintenance costs associated with the time and labor of shutting down the system to replace filters reduces the cost difference as compared with other consumables.

There are many options for filter fabrics, including woven versus felted, surface treatment, fabric weight, pleated or non-pleated, membrane-backed fabrics with or without a catalyst, or solid filters ${ }^{[22]}$. Woven fabrics are made up of warp, the main thread running lengthwise in the weave, and the fill, the thread lacing the warp together. There are all sorts of combinations and customizations, with woven fabrics being stronger and more lasting than felted fabrics. Woven 
fabrics can also become loose and allow gaps between the warp over time, allowing for particles to escape. Felted fabrics are thicker and have randomly oriented fibers, which increase the number of targets and introduce a more tortuous path for particle diffusion time than that of a weave. Depending on the fiber material, different surface treatments can be applied to overcome weaknesses of the base material. Fibers can be coated in silicone, graphite or fluorocarbon to increase abrasion resistance and acid resistance, while inorganic coatings on silica fibers can increase temperature resistance ${ }^{[22]}$. Filter materials with higher weight have greater physical strength and greater target area for particle interaction. This increase in filter fabric density also increases the pressure drop across the filter and the cost of the filter material. Pleated filters greatly increase the surface area per cross sectional area of the filter and pleats can slightly increase the filter's rigidity. Membrane backed fabrics include a membrane on one side of the filter fabric that can have extremely fine diameter fibers to act as a primary target ${ }^{[22]}$. This membrane can be added to reduce cake buildup or to increase the life of the main filter material and tends to have a lower pressure drop than the bulk material ${ }^{[22]}$.

Solid candle filters are rigid and do not deform during the backflow or pulsing process. These filters can be made of many materials and can either be monolithic or composite. Monolithic ceramic filters can be made of clay-bonded silicon carbide, aluminum oxide particles or silicon nitride. Composite ceramic filters may be built with continuous fibers of one inorganic material, with discontinuous filtration fibers added to fill in the matrix. Ceramic filters are commonly used in high temperature applications from approximately $900{ }^{\circ} \mathrm{C}$ to $1100{ }^{\circ} \mathrm{C}^{[22]}$. These rigid filters operate at high temperature and are susceptible to thermal and mechanical shock. The design of solid ceramic filters must consider this to avoid failure.

\subsubsection{Scrubbers}

Wet scrubbers are another method for particulate removal. The primary collection mechanisms for wet scrubbers are impaction and interception. In application, a wet scrubber introduces fine liquid droplets as the targets into the carrier gas. These droplets either contact particles and capture them or increase the overall size of the particles. As particles impact or intercept scrubber liquid droplets and increase in size, gravity settling causes the droplets to fall down to the base of the scrubber where they collect and flow into the reservoir. The droplet liquid used is often water with additives to also absorb other contaminants in the gas stream. 
Wet scrubbers capture particles in the liquid droplets, removing them from the gas stream and preventing re-entrainment back into the gas stream, which is possible with dry particle collection techniques. This process is most effective with particles in the range of $0.1 \mu \mathrm{m}$ to $20 \mu \mathrm{m}^{[22]}$.

Disadvantages of wet scrubbers include freezing of the carrier liquid in winter and corrosion of internal parts due to the continuously wet environment. Corrosion can occur either from acids being absorbed into the carrier liquid and coating all of the internal components, or from materials exposed to the carrier liquid without any corrosion protection mechanism. The addition of sacrificial anodes, coatings of parts, and the use of corrosion resistant materials will greatly increase the life of equipment.

The concept of creating a mist of scrubber liquid and finding a way to maximize contact time is used for a variety of designs. There are vertical spray towers, packed bed scrubbers, impingement plate scrubbers, cyclone spray towers, venturi scrubbers, and many more ${ }^{[22]}$. These five basic designs will be described here.

Vertical spray towers operate by spraying the carrier liquid from the top of the tower while the gas stream flows in from the bottom of the tower. This countercurrent flow increases the chances for particles to interact with the liquid droplets. This design depends heavily on the process of increasing particle size to increase settling velocity. The particle settling velocity must exceed the upward gas velocity for particles to settle out of the gas stream. At the base of vertical spray towers, a drain allows for the particle-laden liquid to be collected and the particulates removed. A mist eliminator is commonly installed at the top of the spray tower to prevent liquid droplets from leaving the tower in the exhaust. Within the tower, there are many levels of spray nozzles. These towers are capable of removing particles from high concentration gas streams and are approximately $70 \%$ efficient in removing particles over $10 \mu \mathrm{m}$ and less efficient for smaller particles ${ }^{[27]}$.

A packed bed scrubber is similar to a vertical spray tower with the addition of some sort of media packed into the majority of the tower below the spray generator. The spray comes in through the top and flows over the packed material. This design creates a scrubber bed with high surface area and a highly tortuous flow path thereby maximizing contact time with the gas. This system is mainly used for sorption of gas phase contaminants in the gas stream. The tortuous path of the packing media acts similarly to a filter media and can pick up particulates as well. 
Impingement plate scrubbers have perforated plates spaced throughout the body of the tower with the carrier liquid flowing across them, down to the drain at the base as illustrated in Figure 4. The gas flows up from the bottom through the perforations in the plates to contact the passing carrier liquid. Spray nozzles are sometimes added below the plates to introduce more droplets before or between plates to maximize the time for particle and droplet interaction. The top of the impingement tower has a mist eliminator often in the form of an axial mist eliminator or packed mesh layer.

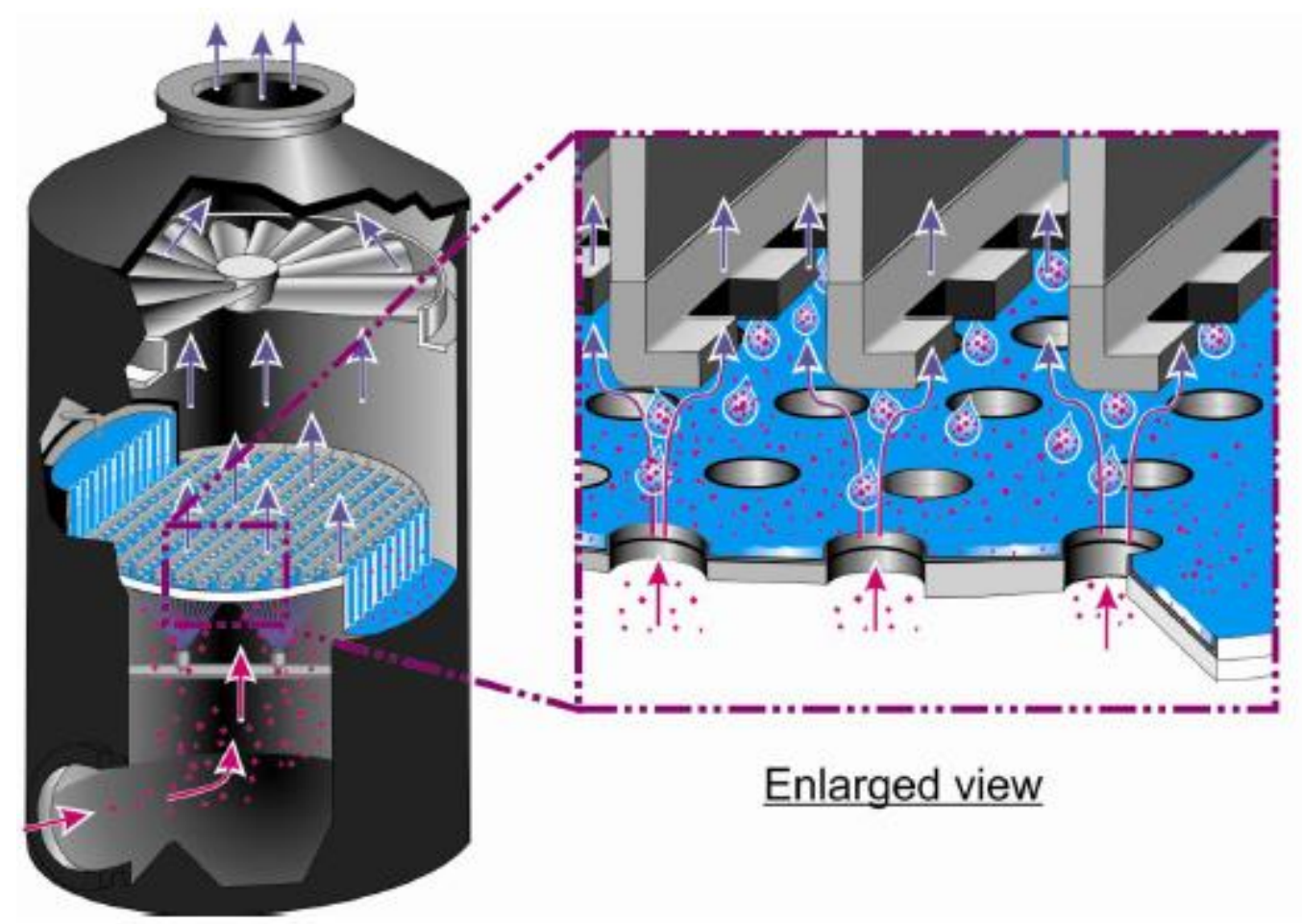

Figure 4: Impingement spray scrubber internal components and capture mechanism ${ }^{[27](\text { Figure 1.23) }}$.

A cyclone spray tower is a cyclone particle separator with a carrier liquid injected into the gas stream. The injected liquid causes particles to group together, increasing their mass. Higher mass particles carry more momentum and contact the cyclone walls quicker. The carrier liquid helps to wash particles away from the walls of the cyclone and can be easily collected in the reservoir. Wet cyclone spray towers can remove particles with particles greater than $5 \mu \mathrm{m}$, which is an improvement from the lower range of $10 \mu \mathrm{m}$ particles removed in the dry cyclone.

Venturi scrubbers use the venturi concept of decreasing the cross sectional area of the gas stream to increase the velocity and decrease the pressure to inject a carrier liquid in this 
constricted section (see Figure 5). This decrease in pressure can be used to pull the carrier liquid into the gas stream and the increased velocity can help atomize the spray pattern. As the velocity is increased, induced turbulent gas-phase flow increases the chances for particles to interact with the carrier liquid droplets. The particle-laden droplets are then either removed by gravitational settling or some other inertial separation process. Venturi scrubber systems have no moving parts and often include corrosion resistant or acid resistant coatings improving their operating life.

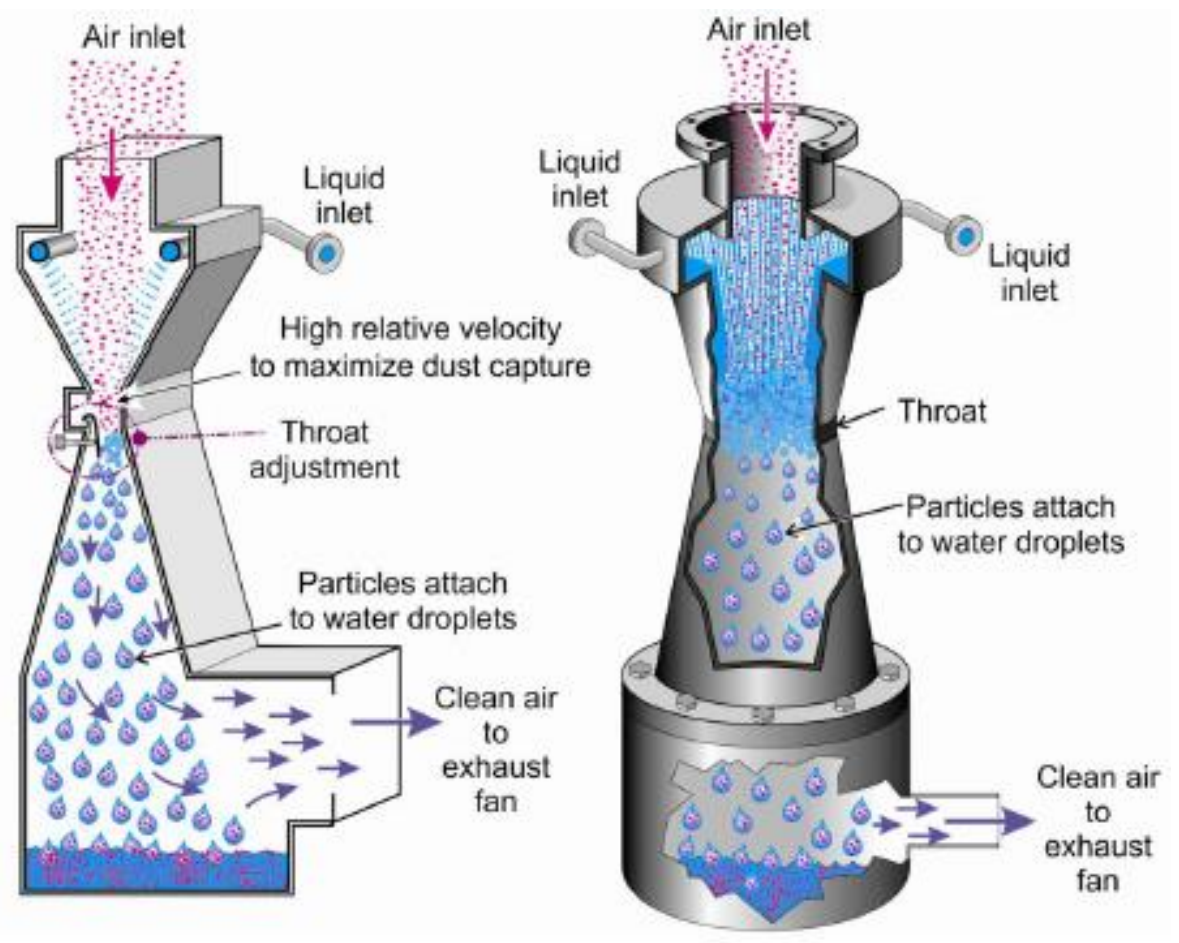

Figure 5: Venturi scrubber design possibilities, components and capture mechanism ${ }^{[27](F i g u r e ~ 1.22) . ~}$

\subsubsection{Electrostatic Precipitators}

Electrostatic precipitators generate non-thermal plasma to charge the particles in a gas stream. Particles will either collide with those charged ions or become charged themselves from the resident high voltage. The systems include oppositely charged collection plates positioned closely together allowing charged particles to migrate toward and stick to the collection plates as suggested in Figure $6^{[22]}$. The particles are then knocked off the plate, or washed away in a wet system, and collected.

Electrostatic precipitation has operational advantages over local exhaust ventilation systems; however electrostatic systems often have a higher initial cost. There are no moving parts to wear 
out in an electrostatic system and the particles are easily collected and removed. Electrostatic precipitators can operate at various temperatures as all the components can be metal. This process can achieve $90 \%$ particulate removal efficiency ${ }^{[22]}$. Gases with high resistance require higher voltages to charge the particles for removal. Additives such as $\mathrm{SO}_{3}, \mathrm{NH}_{3}$ or $\mathrm{NH}_{4} \mathrm{SO}_{4}$ can be injected into the gas stream to reduce particle resistance while increasing the particulate volume to be removed ${ }^{[22]}$. These systems must also be designed to ensure sufficient time and distance for particle migration from the center of the gas stream to the collection plates. There is also a constant high voltage applied to the system to generate the non-thermal plasma, which needs to be consistent in order to be effective. The voltages used are mostly between $20 \mathrm{kV}$ and $100 \mathrm{kV}^{[22]}$.

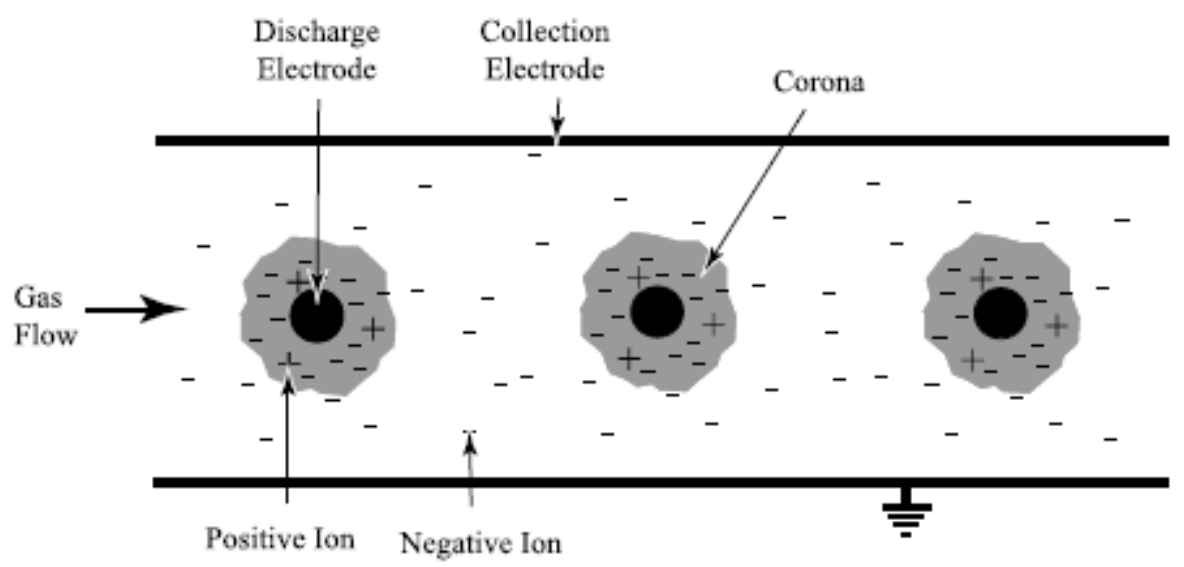

Figure 6: Electrostatic precipitator charge production and particle collection mechanism (Figure 24.2, ${ }^{[22]}$ ).

For this small scale system, a cyclone particle separator as well as a packed bed scrubber were used for particulate control. The cyclone will remove larger particles and the scrubber will remove smaller particles downstream. The cyclone will reduce the particle load of the scrubber as well as the frequency of particle removal from the scrubber reservoir. The packed bed of the scrubber will provide a highly tortuous path for the particles to move through, greatly increasing the chance for contact. The scrubber liquid will then wash collected particles out of the packed bed and into the scrubber reservoir. 


\subsection{Lead}

Lead was added as a criteria air pollutant when leaded fuels were still in widespread use. For example, with lead as a component in automotive fuel, it commonly partially deposited on the cylinder walls in internal combustion engines with the remaining lead released as particulates in the system's exhaust. In a study in the United Kingdom, between $10 \%$ and $58 \%$ of the lead particles generated by burning leaded fuels were deposited on the ground within $100 \mathrm{~m}$ of the roadway, and the rest were small enough to remain airborne ${ }^{[34]}$. During the time leading up to the Clean Air Act, there were lead particulates in the air and possibly gaseous lead compounds. As leaded fuels were phased out, the particulates released greatly decreased.

Lead is still released into the atmosphere by other sources besides leaded fuels. Waste incineration and the burning of coal can both release lead into the atmosphere and can be traced using stable lead isotopes ${ }^{[35],[36]}$. In the United States, there are still plenty of lead processing industries that have airborne lead emissions, including lead smelting, battery manufacturing, and foundries making alloys that include lead ${ }^{[37]}$. These industries mainly produce multi-metal

oxides and sulfates of lead as particulates of varying sizes ${ }^{[38]}$. As airborne lead seems to mainly form particulates, the previously described particulate removal processes all apply to airborne lead control. 


\subsection{HAZARDOUS AIR POLLUTANTS}

According to the U.S. EPA, hazardous air pollutants (HAPs) include any airborne pollutants that are known to cause cancer or other serious health effects. As of 2020, the EPA list includes 187 hazardous air pollutants consisting of mostly organic compounds along with some metal compounds ${ }^{[39]}$. With this many compounds to control, it is simplest to treat them in groups and approach each source of emissions on a case-by-case basis.

\subsection{Dioxins and Furans}

The toxic equivalency factors (TEFs) for PCDDs and PCDFs range from 1 to 0.0003 and 0.1 to 0.0003 , respectively ${ }^{[40]}$. The most toxic of the PCDDs are 2,3,7,8-tetrachlorodibenzo-p-dioxin and 1,2,3,7,8-pentachlrorodibenzo-p-dioxin both with TEFs of 1, which is the top of the TEF scale $^{[41]}$. Compounds with less than 1 are less toxic, making the two mentioned PCDDs the most toxic compounds on the scale. The most toxic PCDF is 2,3,7,8-tetrachlorodibenzofuran with a TEF of 0.1. Due to the high toxicity of these compounds, it makes sense to have them on the HAPs list to regulate their release into the atmosphere.

Dioxins and furans form by two mechanisms. The homogeneous mechanism occurs between $500^{\circ} \mathrm{C}-800^{\circ} \mathrm{C}$ and involves the rearrangement reaction of chlorophenols and chlorobenzenes ${ }^{[41]}$. The heterogeneous reaction occurs in the post-combustion zone between $200^{\circ} \mathrm{C}-400^{\circ} \mathrm{C}$ and involves the reaction of chlorophenols and chlorobenzenes catalyzed by fly ash ${ }^{[41]}$. Both of these reactions require the presence of chlorophenols and chlorobenzenes, which are not stable under high temperature plasma arc conditions ${ }^{[42][43]}$. Eliminating the precursors in the gas stream or the time in the temperature zones where these reactions occur can effectively control dioxins and furans. Chlorophenols, chlorobenzenes, dioxins and furans all degrade over approximately $900^{\circ} \mathrm{C}$ (Figures 7 and 8$)^{[42]}$. For this reason, process gases are often kept at high temperature until a quench, which rapidly cools the gases through the temperature range of reaction and recombination. 


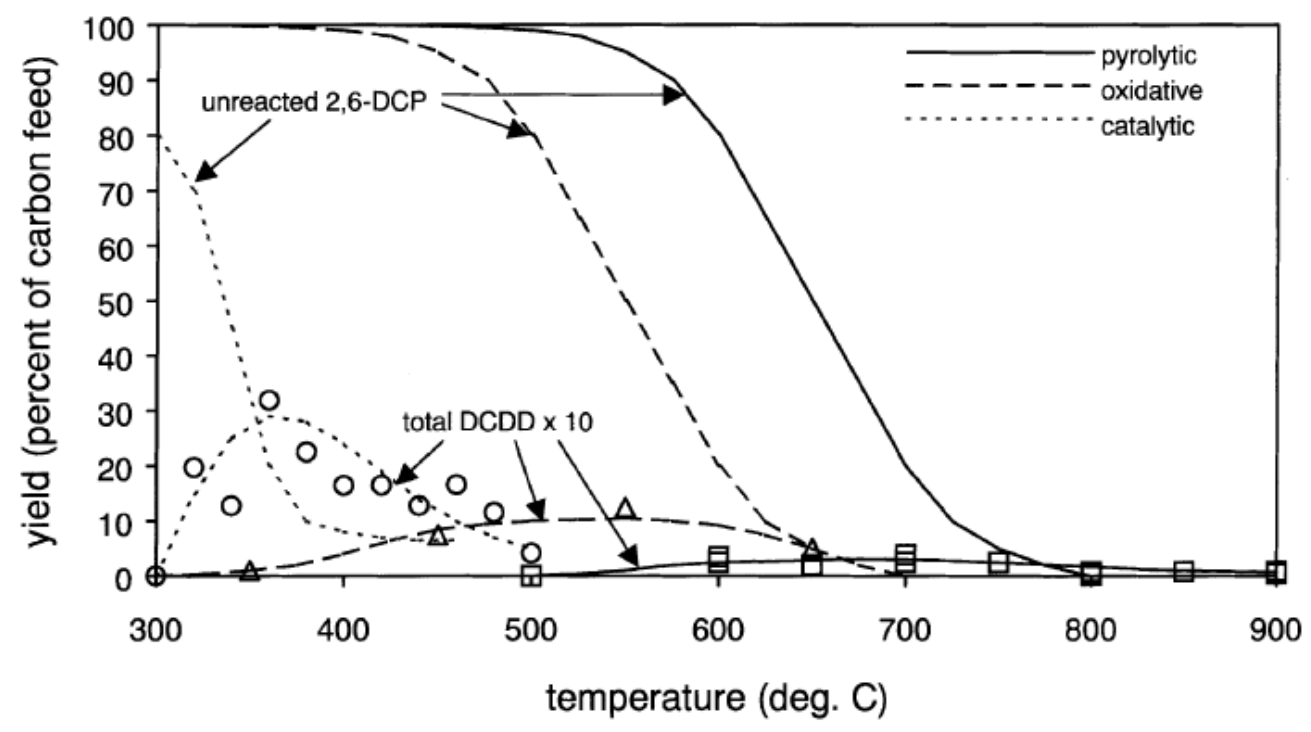

Figure 7: DCDD production from 2,6-dichlorophenol (2,6-DCP) at different temperatures.

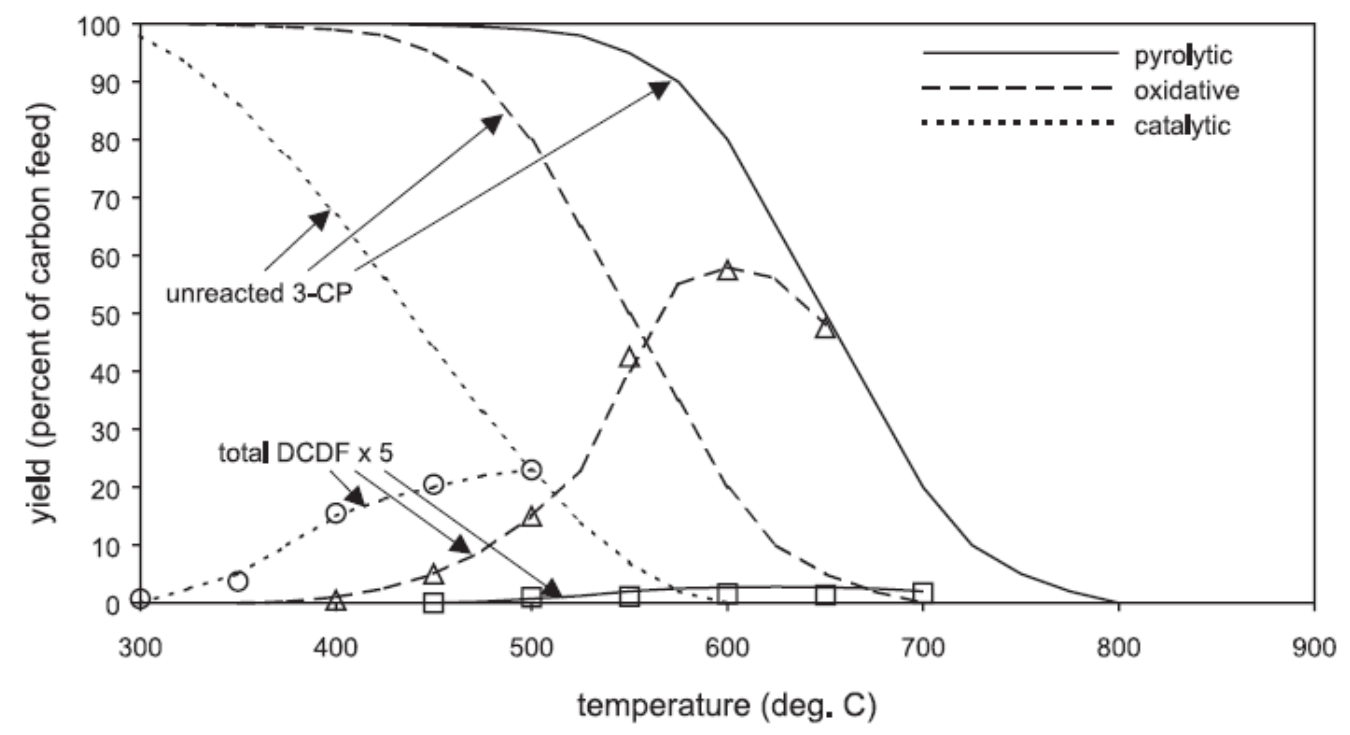

Figure 8: DCDD production from 3-chlorophenol (3-CP) at different temperatures.

\section{$\underline{4.2 \text { Chlorine compounds }}$}

Many of the organic molecules listed as HAPs contain chlorine atoms in their molecular structure, including allyl chloride, benzotrichloride, bis(2-ethylhexyl)phthalate (DEHP), bis(chloromethyl)ether, carbon tetrachloride, and many more ${ }^{[39]}$. During high temperature processing like that occurring in plasma gasification, most organic molecules are broken down and the chlorine atoms can be released to freely interact with any other nearby molecules. Free 
chlorine can be treated similarly to sulfur, as both are water soluble and form acids in scrubber solutions.

\section{$\underline{4.3 \text { Metals }}$}

Antimony, arsenic, beryllium, cadmium, chromium, cobalt, manganese, mercury, nickel, and selenium are all metals listed as HAPs ${ }^{[39]}$. These metals come from various sources including manufacturing, transportation emissions, and metal production industries. Industrial processes that release metals mainly do so in the form of metal oxides as particulates. When welding galvanized steel or any zinc containing steels, zinc oxide is released and can range from $0.03 \mu \mathrm{m}$

to $0.3 \mu \mathrm{m}^{[32]}$. Zinc oxide, along with some other metal oxides can cause metal fume fever, which is often severe enough to put the welder out of work for a few days. As the majority of the metals that are released from MSW in plasma gasification are in the form of metal oxide particulates, the particulate control techniques previously described apply. 


\subsection{PROTOTYPE PLASMA GASIFICATION SYSTEM AND DESIGN COMPONENTS}

The goal of this project was to design a low capital cost plasma gasification system to process municipal solid waste while producing minimal regulated emissions, using few to no consumables, that could be delivered to parts of the world that do not have effective and sustainable waste management systems in place. This project involved designing and building the majority of the components, and making use of components that could be purchased at low cost.

The components that were built include the plasma torch, cooling system, gasification chamber, cyclone particle separator, scrubber, scrubber reservoir, sensor system and the system's frame (Figures 9 and 10). The MSW enters through a small tube in the gasification chamber lid. There are two ports at the base of the gasification chamber for excess molten metals and glass to be released. A cooling system is connected to the plasma torch to prevent it from overheating. The gases then travel to the particle separator where larger particles are removed. After the particle separator, a catalytic converter encourages oxidation and reduction reactions of the gases. The gases then travel into the scrubber, which has a reservoir where acids, fine particulates, and heat are removed from the system. After these emission controls, the gases pass by the sensor bank where levels of select constituents can be measured and recorded.

This section describes the design and fabrication of the components used in the prototype plasma gasification system from the plasma torch to the sensor system in the direction of gas flow (see Figure 9). The details of torch design along with the challenges of electrode material selection and process gases are explained. The construction, function, and issues associated with the particle separator, catalytic converter and packed column scrubber are each described. The power supplies used for the torch and each of the pumps are outlined. A description of the frame and its construction is also included. 


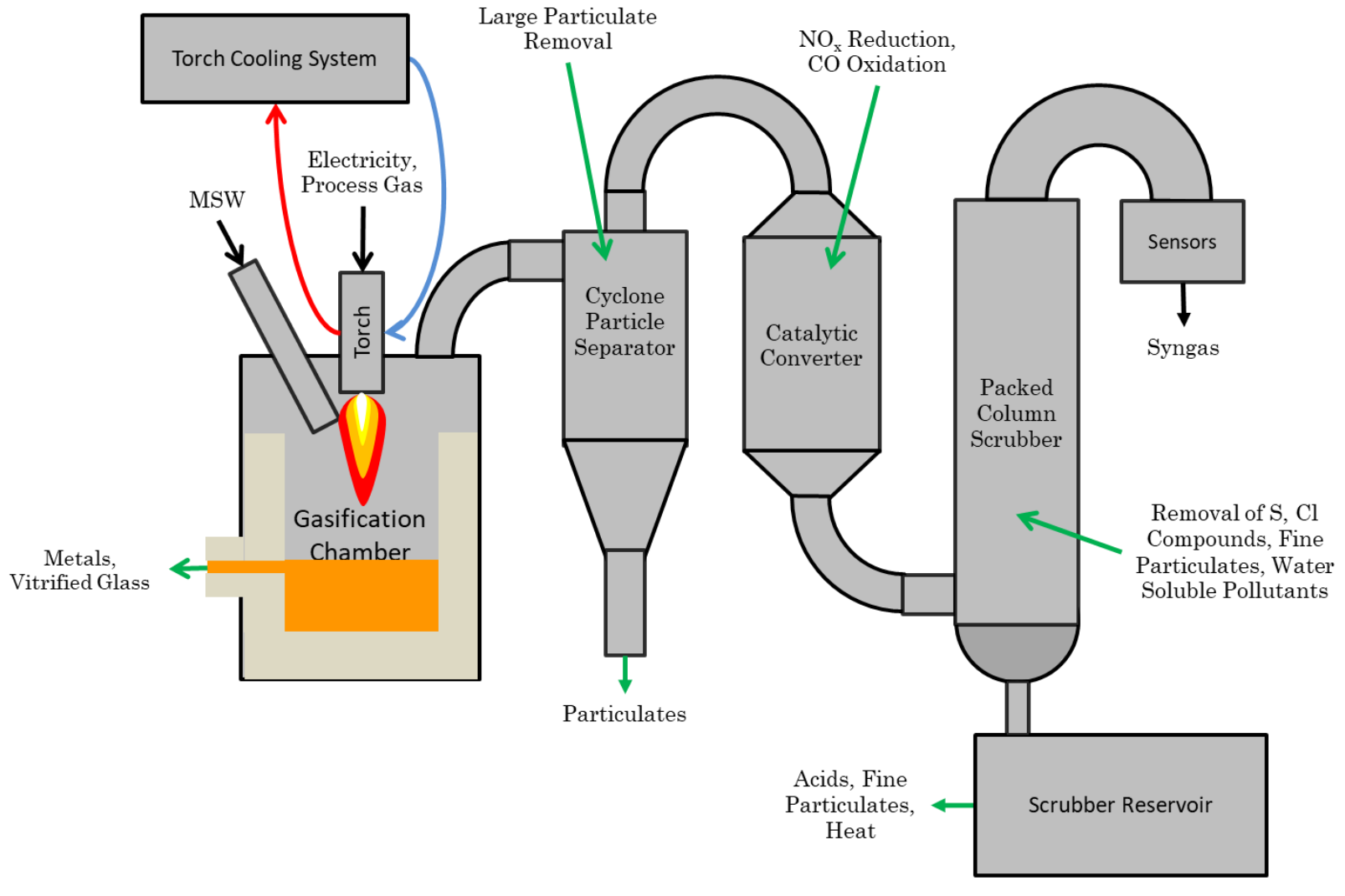

Figure 9: Schematic of plasma gasification system components showing inputs and outputs. 


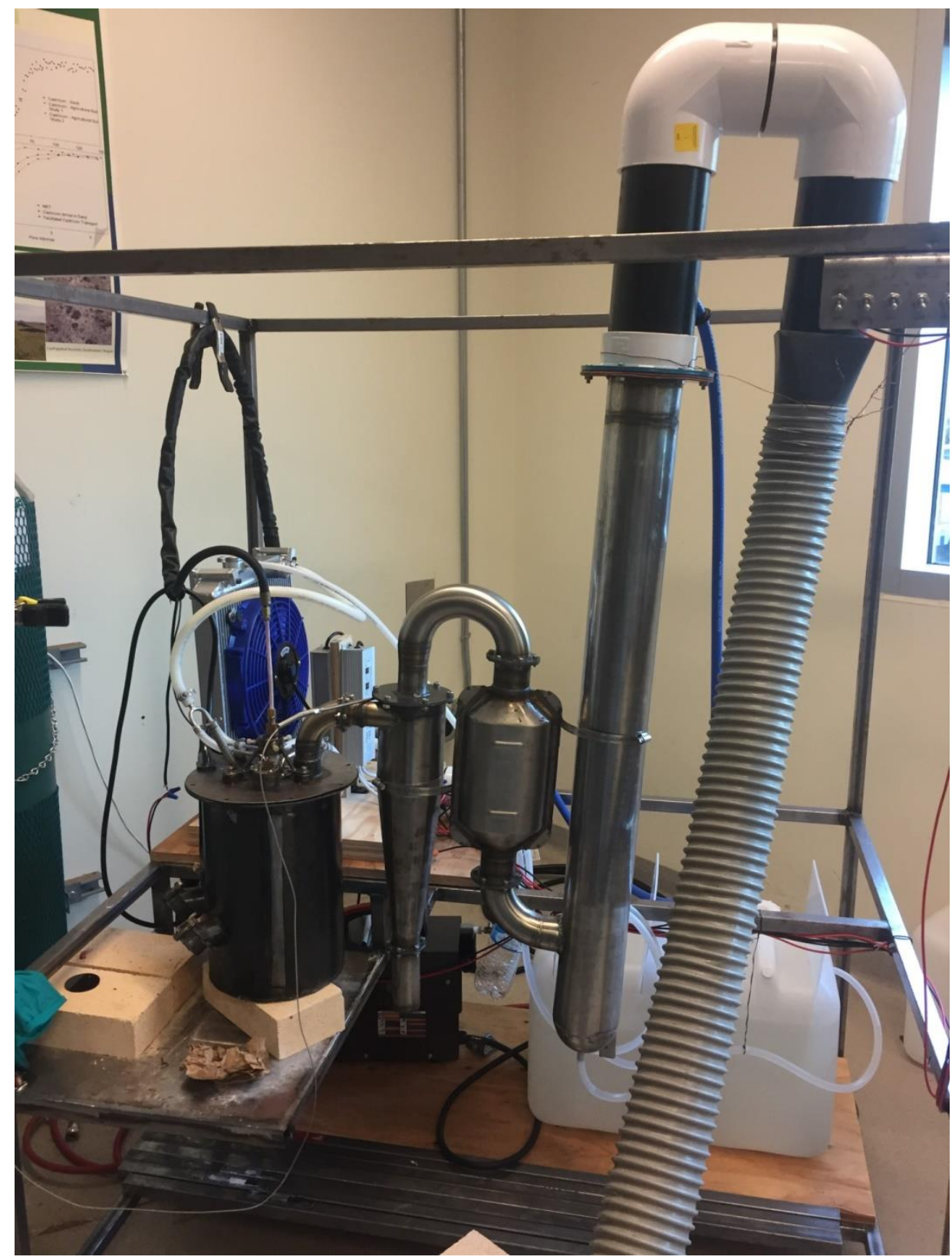

Figure 10: Prototype plasma gasification system in the lab.

\subsection{Plasma torch}

The plasma torch used in this system is a non-transferred arc plasma torch with a water jacket for cooling. The torch has five components that are contained within an alumina insulator tube, which can be inserted and removed from the water jacket (Figure 11). Starting at the connection for air and gas is a fitting that has a 1/8-inch NPT thread for connection with a pressurized gas line on one end and a 1/4-20 threaded hole for the electrode collet on the other end. There are four holes drilled at $90^{\circ}$ intervals along the circumference of the $1 / 4-20$ hole to allow gas flow past the 
collet while providing enough electrical contact on the threads for current to flow. There are also two O-rings in slots at the base of this fitting to seal the back of the alumina tube and prevent loss of gas pressure. These O-rings also provide friction to allow the spacing between the two electrodes to be adjusted. The electrode collet was made from a 1/4-inch diameter copper rod with a 1/8-inch hole for a 1/8-inch diameter negative electrode to be inserted. The vortex generator can slide down the collet to increase pressure on the electrode and close the collet onto the electrode to hold it in place. The vortex generator also causes the gas passing through to form a spiral vortex to help center the plasma as it leaves the nozzle. The negative electrode is centered within the alumina tube with the vortex generator and the base of the electrical and gas connection fitting. The water cooling jacket has a $1 / 2$-inch diameter hole for the nozzle to be inserted. When the gas is flowing, the power can be turned on and the current will flow through the electrical fitting to the collet and negative electrode and jump the gap to the positive electrode to form plasma as long as the breakdown voltage of the passing gas is below the applied voltage.

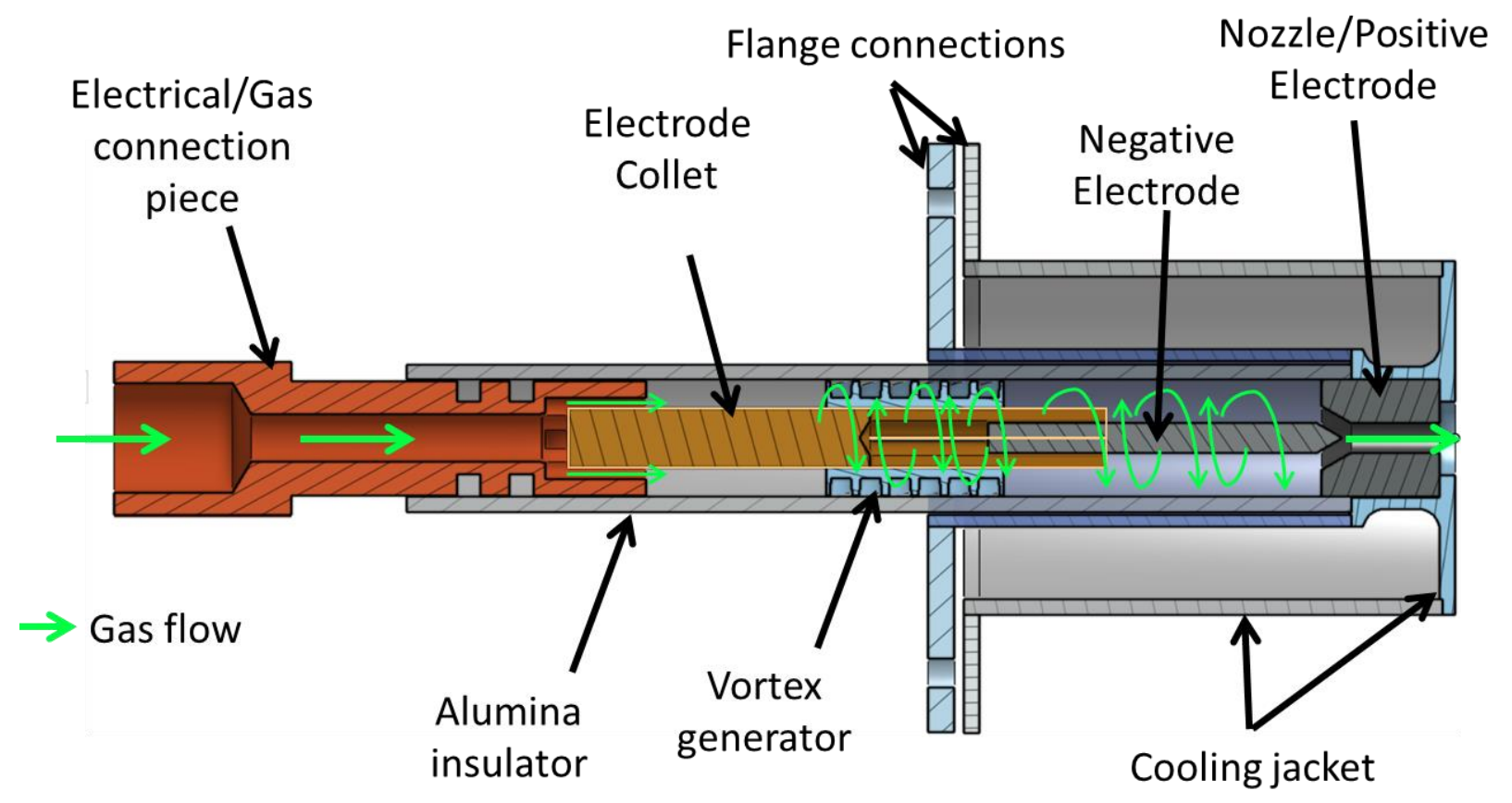

Figure 11: Plasma torch internal components and gasflow path.

The torch was tested in this study by varying the amperage on the power supply and the air pressure to produce plasma, shown in Figure 12. The amperage ranged from 15 to 50 amps in 5 
amp intervals and air pressure from 30 to $75 \mathrm{psig}$. When the air pressure was below $30 \mathrm{psig}$, there was no plasma formed, however there was arcing within the torch. Above $60 \mathrm{psig}$, while plasma did form, gas flow was excessive and wasted. The amperage did not seem to have any visual effects on the size of the produced plasma. The lower the amperage, the closer the electrodes needed to be spaced to overcome the breakdown voltage of the air to produce plasma. Amperage of 35 amps was settled on to maintain consistent electrode spacing for further experimentation.

To estimate the temperature of the plasma exiting the torch, small samples of metal and pieces of glass were tested. In all of these tests, the steel pieces melted immediately. Additionally, in each batch test, the glass pieces melted in less than three seconds. The melting point of silica glass is $1700^{\circ} \mathrm{C}$ suggesting that the plasma exceeded that temperature. Additional and similar tests were conducted to estimate the temperature of the plasma in application (i.e., with the torch mounted in the gasification chamber) as described in section 5.10 System Testing.

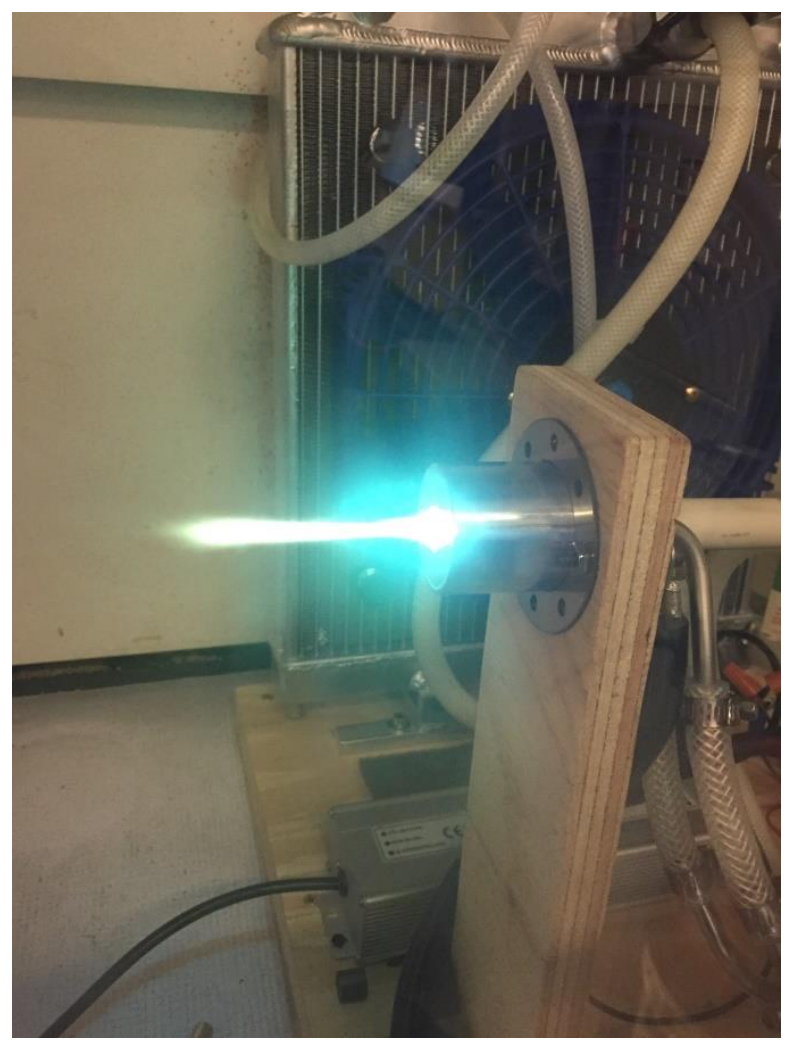

Figure 12: Plasma torch being tested within the fume hood. 


\section{$\underline{\text { 5.1.1 Cooling system }}$}

The torch cooling system consists of a cooling jacket constructed of 304 stainless steel connected through fiber-reinforced silicone tubing to an aluminum automotive radiator modified with custom aluminum hose barbs welded to the openings, all driven by a 1-gpm centrifugal pump (Figure 13). The stock radiator fan was mounted to the radiator and connected to a 12-volt dc-power supply to provide increased cooling if necessary. The coolant liquid tested consisted of a 10:1 ratio of distilled water to ethylene glycol antifreeze for corrosion protection.

The cooling jacket is made of several parts welded together (Figure 14). The interior is a lathe cut end piece welded to a section of 304 stainless steel pipe. This end piece has a $1 / 2$-inch diameter bore for the electrode to slide into, and an opening just under $1 / 2$ inch for the electrode to rest. The inner corners of the end piece are rounded to prevent crevice corrosion and prevent coolant eddies around the edges. These two internal pieces were then welded to a flange that has holes for coolant flow tubes and for mounting to the gasification chamber. There are two inlet and two outlet tubes, with the inlet tubes terminating at the top of the chamber and the outlet tubes terminating close to the end piece where the electrodes produce the most heat. A larger tube section was welded around the exterior to seal off the interior chamber.

As mentioned above, the heat exchanger includes an automotive radiator. Modifications included two hose barb type adaptors cut on a lathe and welded to the inlet and outlet of the radiator. The adaptors allowed $1 / 2$-inch reinforced silicone tubing to be used for the coolant plus an additional $1 / 4$-inch tube connected to the overflow of the radiator. The pump is made of polyetheretherketone (PEEK), allowing it to handle the temperatures of the heated coolant. The pump is also mounted on the downstream side of the radiator to reduce the heat sent through the pump. 


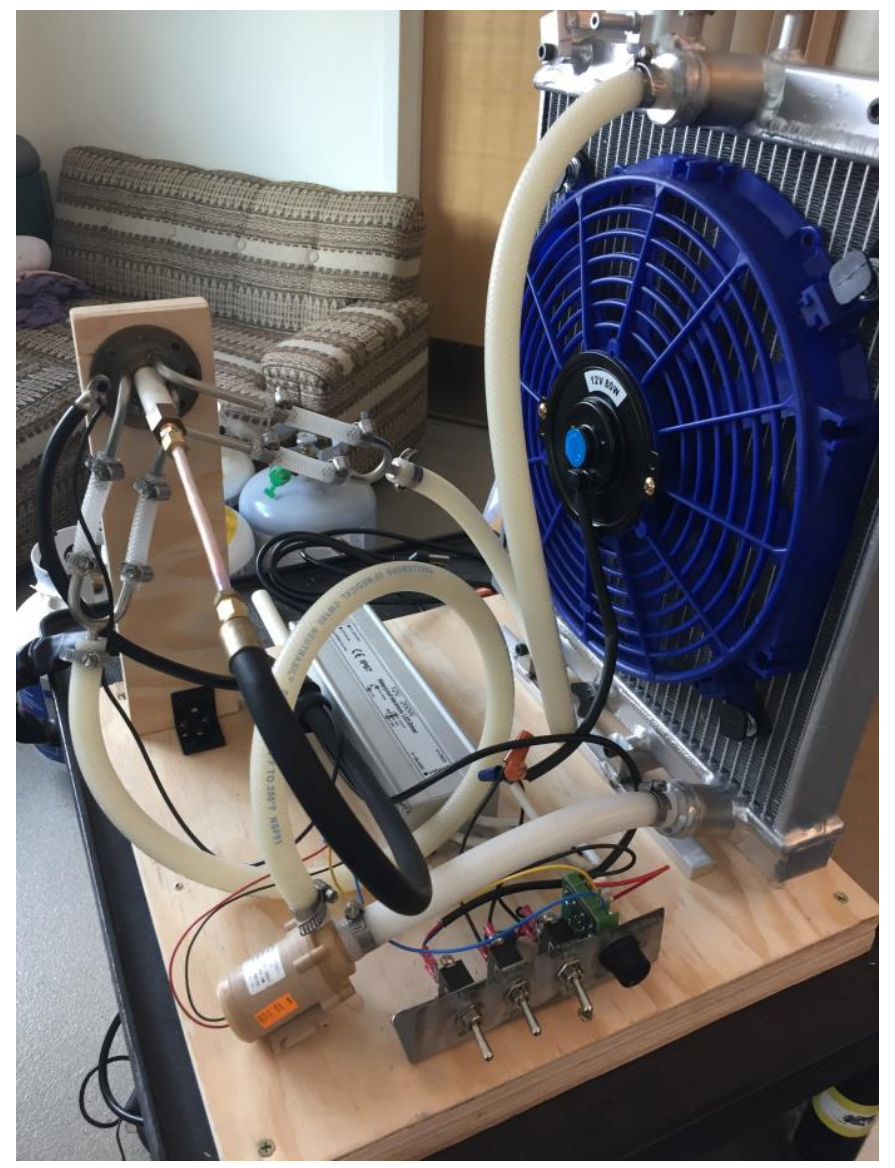

Figure 13: Cooling system with radiator, fan, pump, power supply, and coolant tubes.

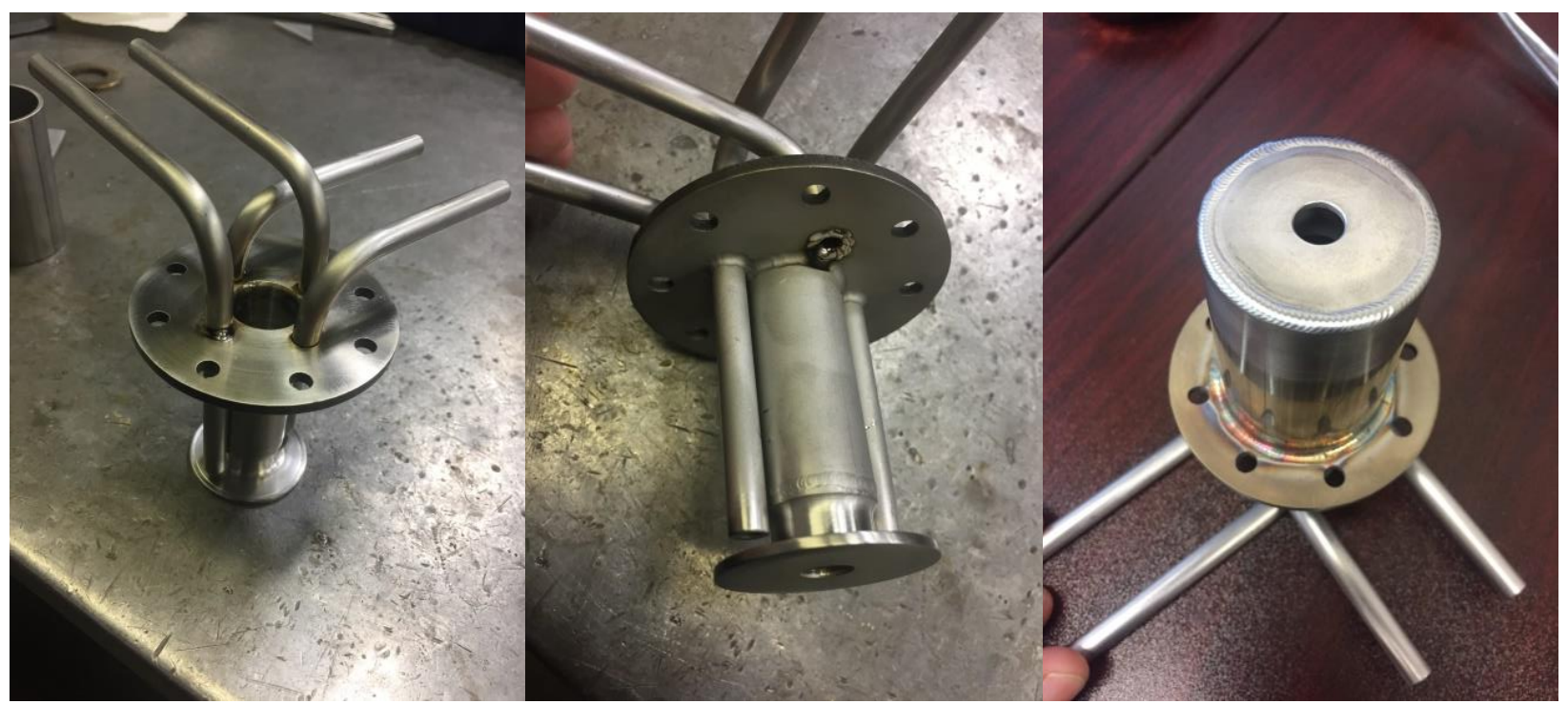

Figure 14: Coolant jacket for the plasma torch. Inlet and outlet tubes connected to the flange (left), internal view of the torch tube, coolant tube connections and end piece (middle) and the gasification side of the completed jacket (right). 


\subsubsection{Electrode Materials}

The initial design included extruded graphite electrodes and compressed air. Testing of these components system created issues that led to a search for alternative materials. For example, the negative electrode would oxidize enough to stop plasma production in less than 30 seconds during the initial testing. Further tests were conducted after modifying the design and spacing of the electrodes, running the plasma for 10 seconds per test. The negative electrodes were initially a solid 1/8-inch diameter rod with the ends cut flat to have an elongated cylinder shape. During initial testing, the sharp edges of these rods were oxidized and disintegrated, leaving a pointed electrode with a flat tip (Figure 15, left, middle, respectively). As the edges of the electrode disintegrated, the gap between electrodes increased. Greater spacing between electrodes requires a higher voltage to ionize the gas to produce plasma. Without increasing the supplied power, this increased gap resulted in the current taking the path of least resistance and arcing to the stainless steel cooling jacket (Figure 15, right).

The next material tested as part of the negative electrode design was tungsten. Tungsten is used for tungsten inert gas (TIG) welding electrodes and is readily available in sizes fitting the torch collet. Tungsten also has the highest melting point of any known metal, which will help to increase the life span of the electrodes.

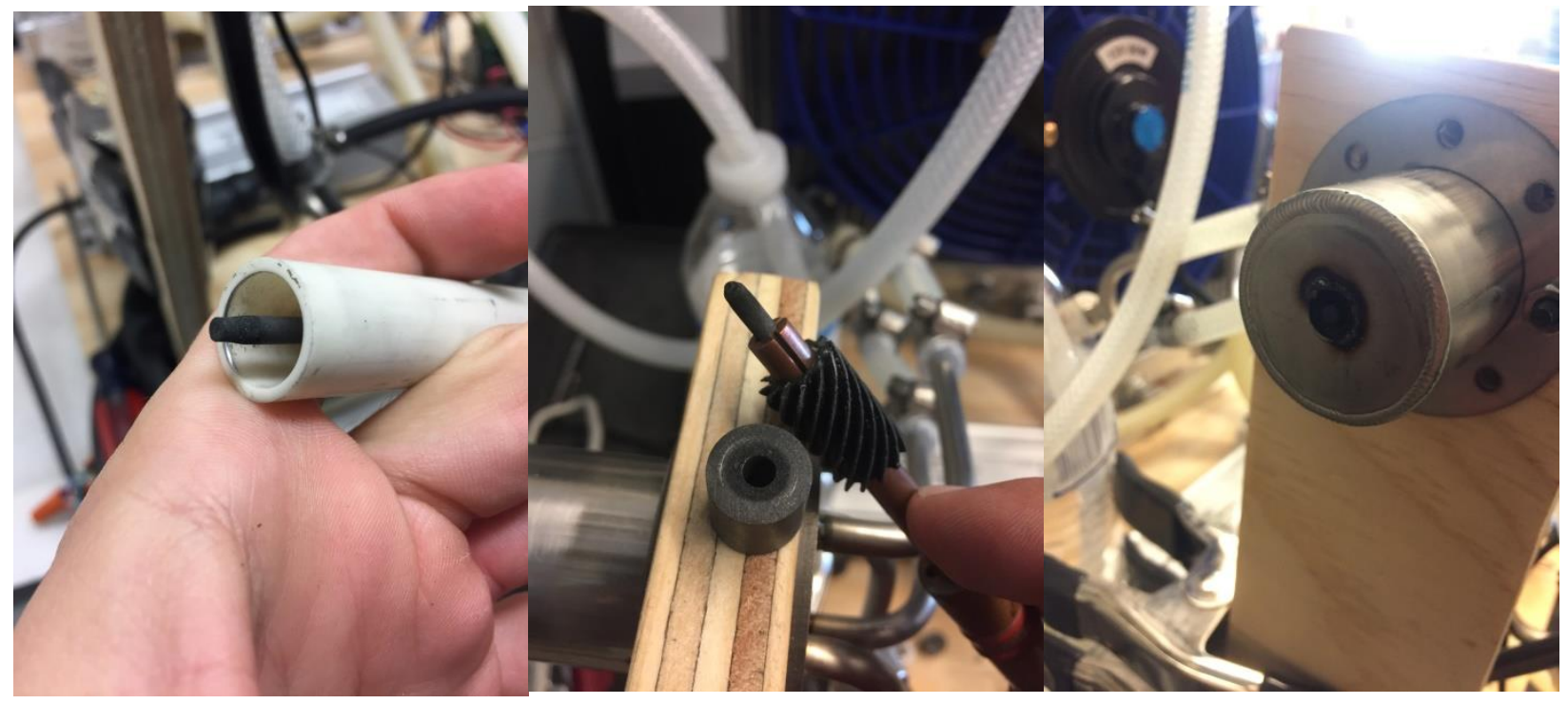

Figure 15: Graphite electrode wear on both positive and negative electrodes (left), negative electrode (middle) and positive electrode with arcing to the cooling jacket. 
The positive graphite electrode did not have nearly as much wear as the negative electrode inside the cylinder. There was, however, minor wear on the exterior face of the outlet side of the positive electrode. Graphite is less conductive than stainless steel at low temperatures, so the plasma was arcing to the stainless steel on the cooling jacket (Figure 15, middle, right). These arcs caused marks similar to what a tack weld would look like without a shielding gas. Some material from the cooling jacket and from the exposed face of the electrode was lost due to arcing. For this reason, the next positive electrode was made out of copper.

The first test using a positive copper electrode and a negative tungsten electrode revealed a different problem with oxidation. The tungsten was oxidizing to form what looked like tungsten trioxide $\left(\mathrm{WO}_{3}\right)$, which coated the negative electrode and spattered the positive electrode enough to form an insulating layer and stop the formation of plasma (Figure 16). The tungsten trioxide formation along with the breakdown of the graphite electrodes suggested that using compressed air as the gas may not be a good choice for this torch. The gas was then switched to compressed nitrogen. A short test showed the oxide layer breaking down completely, leaving the negative electrode as a clean metal surface where the plasma was produced (Figure 16, right). Part of the tungsten was consumed by the oxide formation as evidenced by the pitting on the tip of the clean electrode. The copper electrode was cleaned using fine sand paper to remove the built up oxide layer.

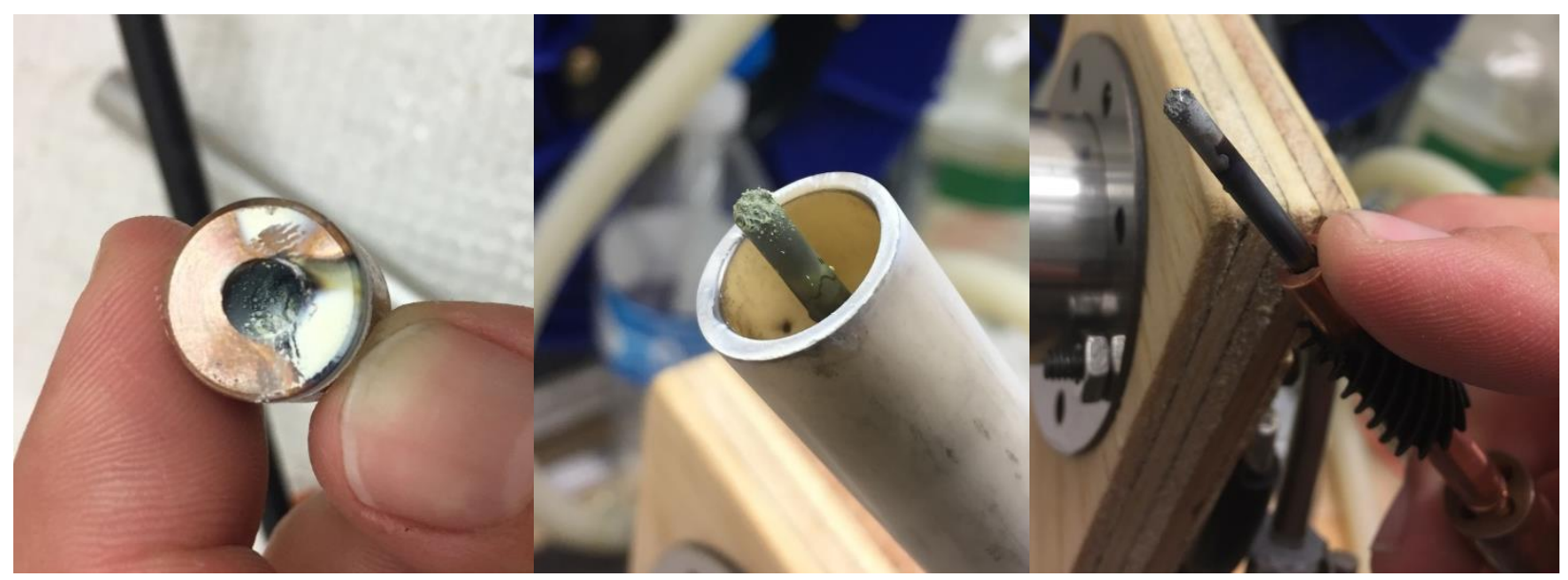

Figure 16: Tungsten trioxide on the positive copper electrode (left) and negative tungsten electrode (middle), and the oxide free negative tungsten electrode (right). 


\subsubsection{Nozzle Design}

Aside from the failure of the original nozzle material, testing of the nozzle indicated that the design was also highly inefficient. The first nozzle used a chamfered 3/16-inch hole through the entire nozzle (Figure 17, a). This nozzle did not increase the air velocity enough to push the plasma out of the electrode. The plasma was forming somewhere between one third and two thirds of the way through the nozzle resulting in arcing and nozzle deterioration. The initial assumption was that a 1/8-inch diameter negative electrode positioned in the center of the opening would allow for a small gap between the two electrodes for plasma formation. The negative electrode could then be positioned at different depths through the nozzle to modify the plasma. This proved to be challenging as the electrode would arc directly to the cooling jacket when it was too far into the nozzle, or it would short to the interior of the water jacket if the negative electrode was positioned too far back.

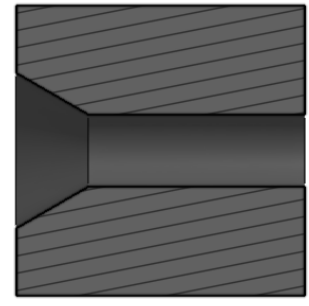

a)

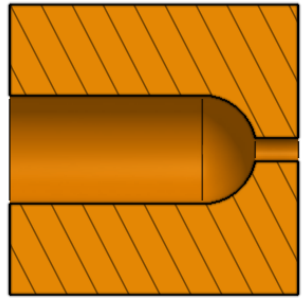

b)

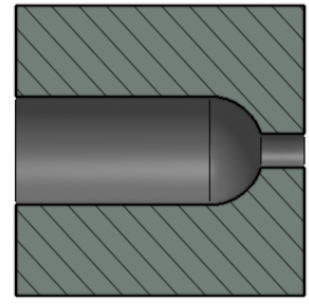

c)

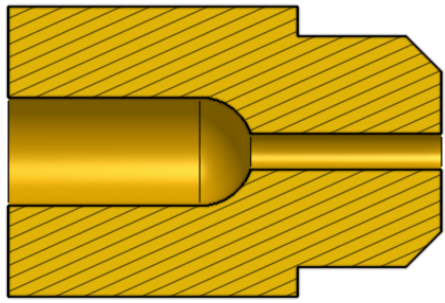

d)

Figure 17: Nozzle designs for the positive electrode. Initial graphite design (a), copper narrow nozzle (b), copper wider nozzle (c) and copper nozzle with extended tip (d).

The second iteration involved changing both the material and the internal design (Figure 17, b). The opening at the end of the nozzle was narrowed to a diameter of 0.040 inches to increase the velocity of the gas. At the base of the large bore is a hemispherical surface to provide a constant gap around the tip of the negative electrode. This would allow for the electrodes to be adjusted once to their optimum spacing and then fixed in place. These changes greatly increased the amount of operation time of the plasma torch, although the small diameter of the nozzle was opened up by the plasma (Figure 18). For example, the first test resulted in a small amount of copper melting and being pushed out of the nozzle and partially depositing on the outer edge of the nozzle (Figure 18, left). After several more tests, the diameter of this hole increased a small amount in an uneven manner; this out of round hole was a challenge to measure (Figure 18, 
right). The other side of the nozzle showed that the negative electrode was not centered as evidenced by the wear on the interior surface of the nozzle (Figure 18, middle).

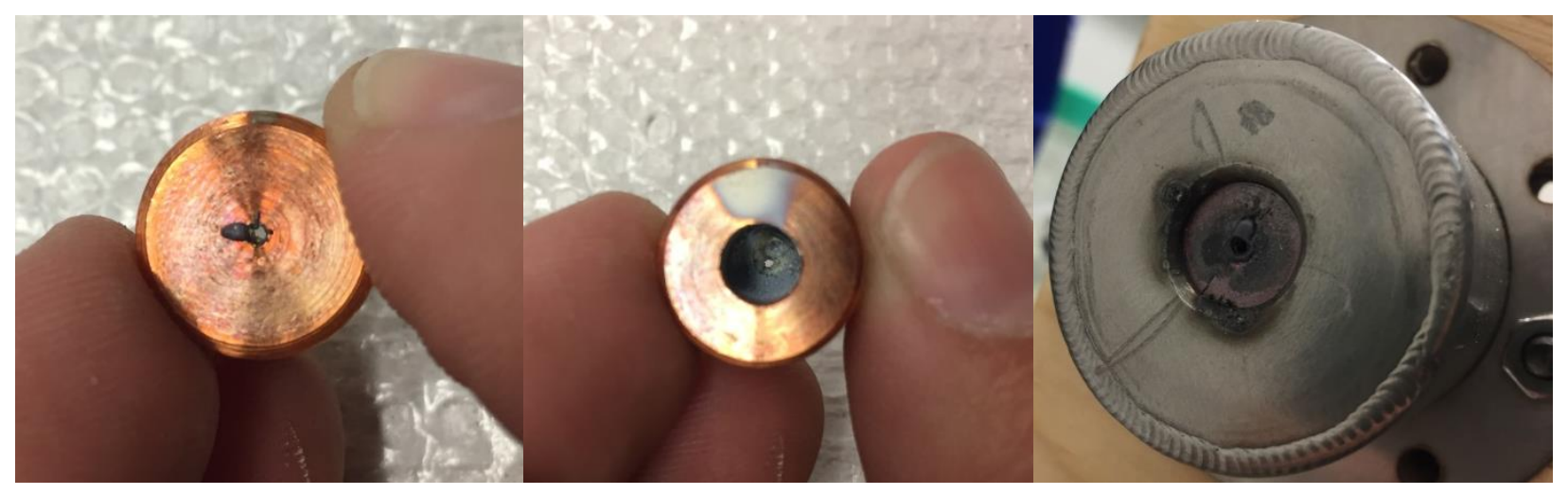

Figure 18: Copper 0.040-in. diameter nozzle after the first test (left, middle) and after several more tests (right).

The third design incorporated a small diameter of 0.060 inches into the positive copper electrode (Figure 17, c). This 50\% increase in diameter no longer had material melting away on the narrow section in the following tests. The relatively short distance between the rounded part of the nozzle and the exit caused the plasma plume to be short, wide, and slightly erratic, suggesting that there was not enough distance in the narrow nozzle section to direct the gas flow into a long slender plume. A fourth design was then made to see if increasing the length of the narrow nozzle section could improve the plasma plume shape (Figure 17, d).

The upstream side of each electrode showed increasing wear further and further from the center of the electrode suggesting that the negative electrode was migrating out of alignment. Upon disassembly and inspection of the torch components, it was discovered that the vortex generator and part of the collet had melted (Figure 19). Furthermore, the collet melted into the vortex generator off to one side greatly affecting the alignment of the tip of the electrode. 


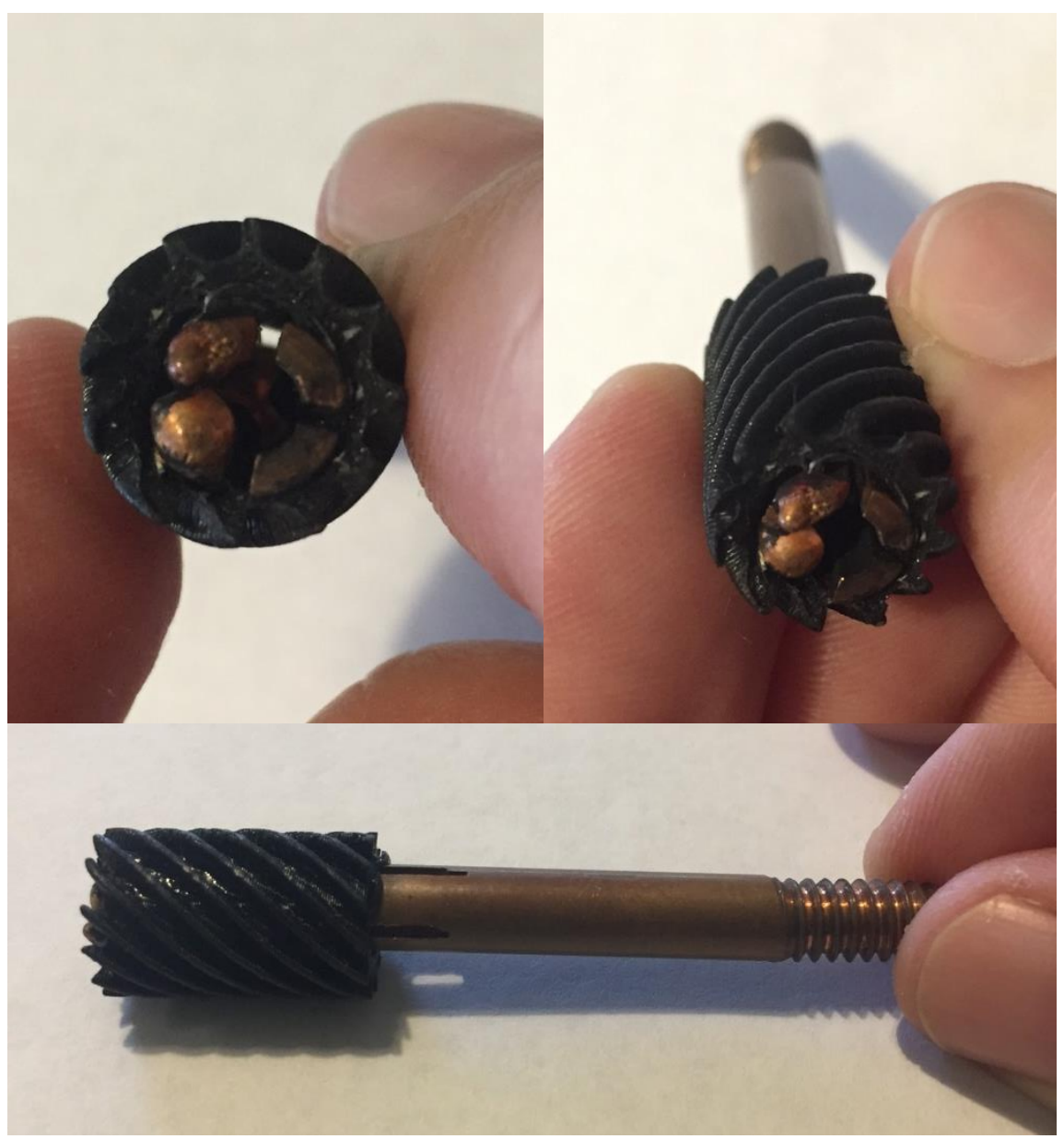

Figure 19: Melted vortex generator and collet.

There was another issue with the alignment of the negative electrode, and that had to do with tolerances of the cylindrical parts. The alumina tube holding the torch components together slides into the cooling jacket. A thin silicone gasket was to be inserted to center the torch, however, installation revealed that the alumina tube was not uniformly round. The alumina tube had an outer diameter ranging from 0.485 inches to 0.511 inches. The out-of-roundness tolerance of the tube is $0.500+/-0.015$ inches, which inhibits the torch components from being centered. To counteract the out-of-roundness of the tube, a centering device was designed and installed on the top of the gasification chamber (Figure 20). This device used three screws to adjust the position of the upper section of the torch to align the electrode. The centering device improved the performance. 


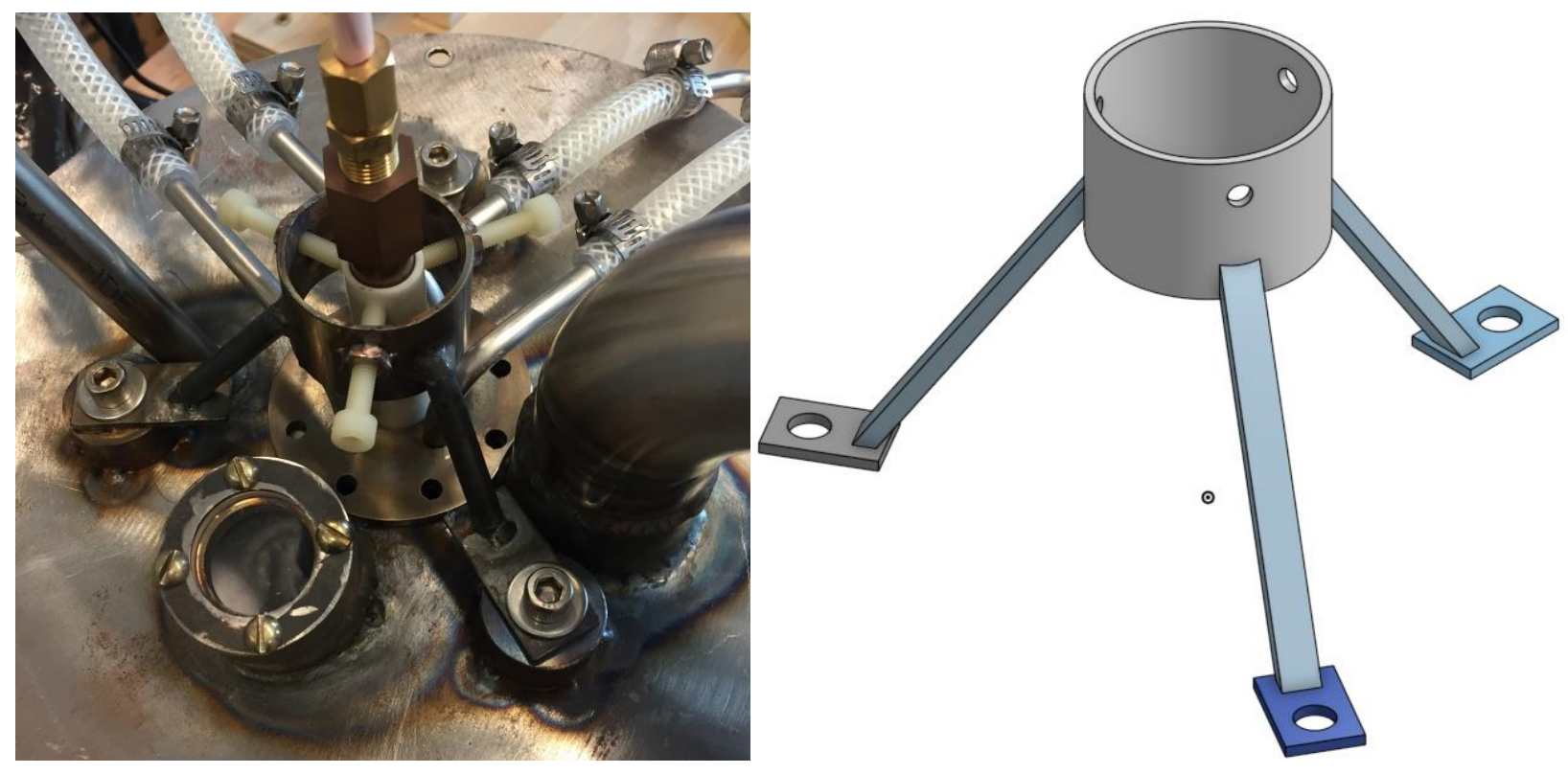

Figure 20: Torch centering device with glass-filled nylon screws mounted to the top of the gasification chamber (left), and the CAD model of the centering device (right).

\section{$\underline{5.2 \text { Gases }}$}

The most commonly used gases for plasma cutting are oxygen, argon, hydrogen, carbon dioxide and helium. The original design of the plasma torch in this prototype included the use of compressed air as the process gas. After testing with different electrode materials, it was found that the process gas needed to have less oxygen to prevent oxidation and eventual destruction of the electrodes. Testing continued using compressed nitrogen gas, however the nitrogen plasma temperature test indicated the torch did not efficiently melt stainless steel.

Maher I. Boulos, of the Institute of Electrical and Electronics Engineers, has studied the characteristics of argon and hydrogen in the range of thermal plasmas. When the enthalpies of these plasma processing gases are plotted as a function of temperature, it shows that argon has a relatively low enthalpy compared to nitrogen (Figure 21). This difference in enthalpy is likely due to the fact that nitrogen gas produces more ions, atoms, and electrons as it dissociates within the plasma (Figure 22). 


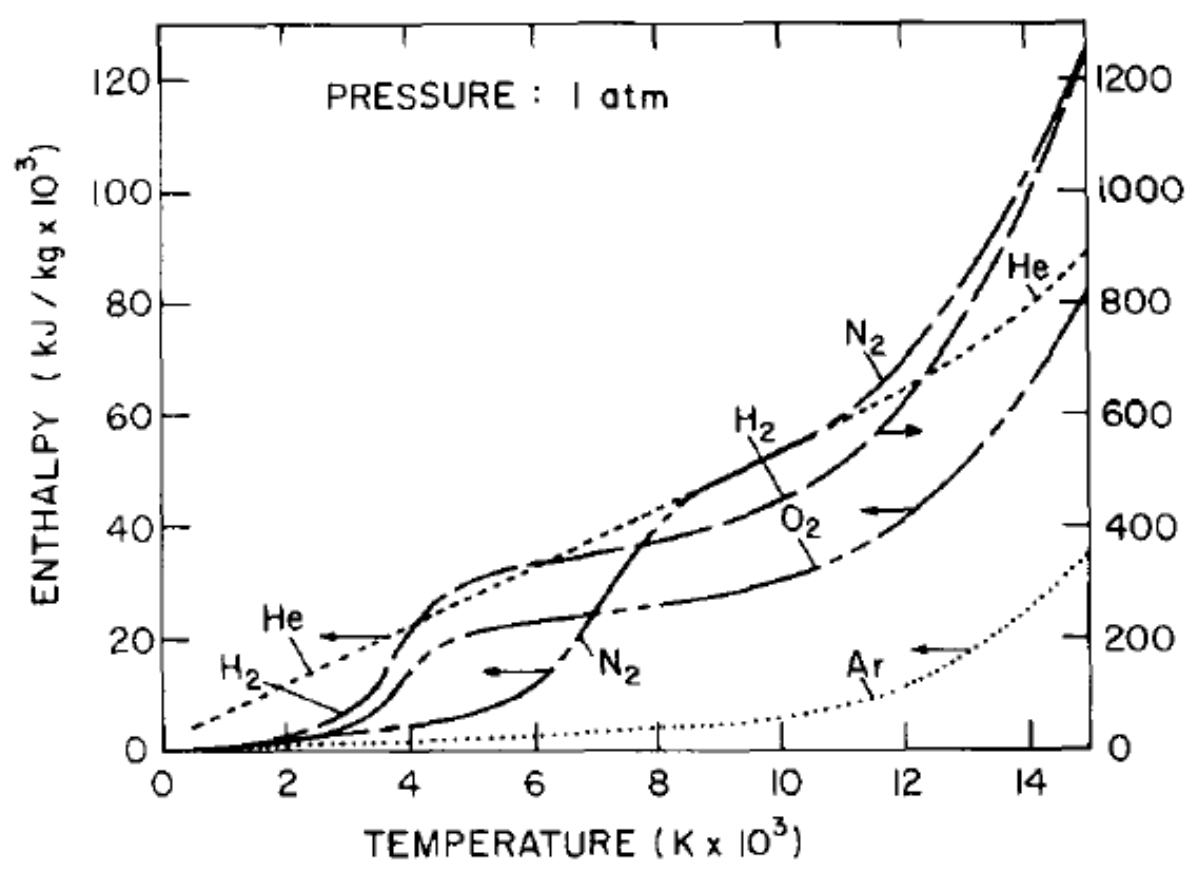

Figure 21: Specific enthalpy for different monatomic and molecular gases as a function of temperature ${ }^{[44]}$.
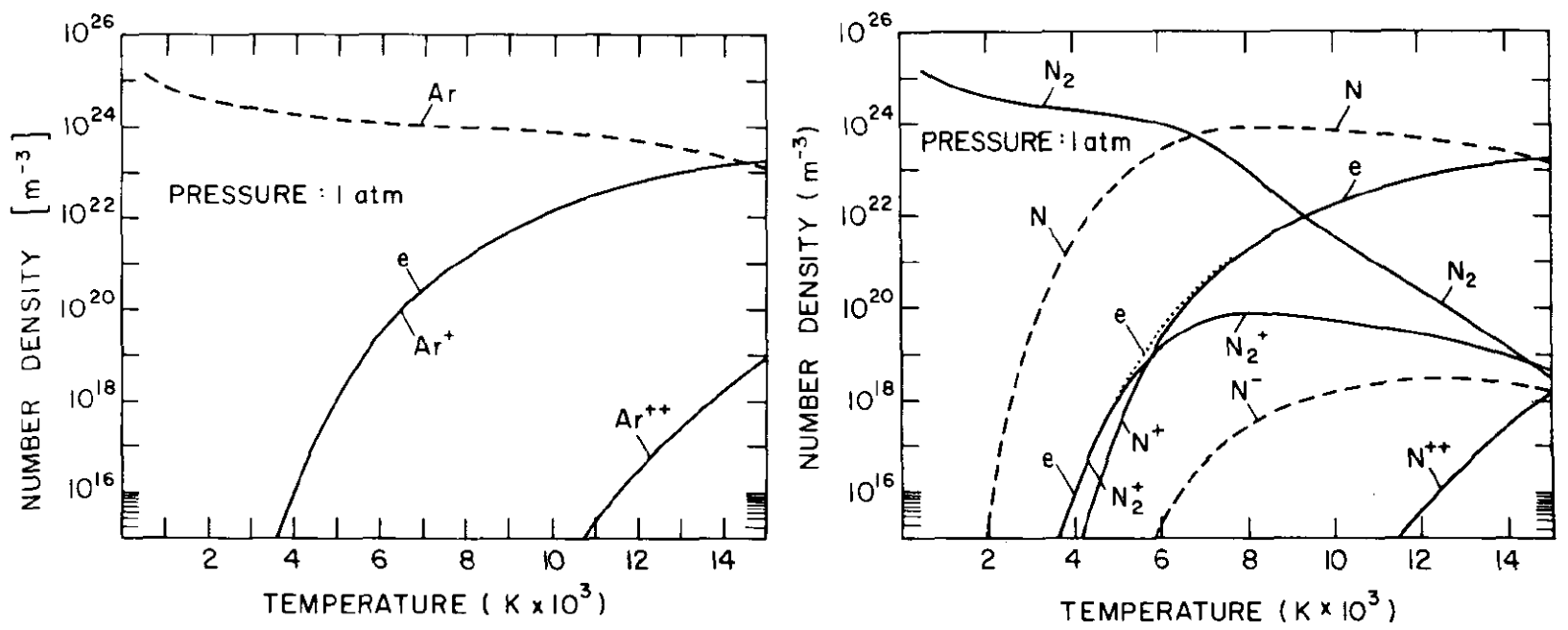

Figure 22: Equilibrium composition for argon gas (left) and nitrogen (right) as a function of temperature ${ }^{[44]}$.

The addition of hydrogen gas to argon greatly increases the thermal conductivity of the gas. The hydrogen will dissociate, introducing more ions and electrons in the plasma, which increases the density of charged particles. Argon has a higher mass than nitrogen, so if the argon/hydrogen mixture could reach a similar energy level, then the argon atoms may have the potential to have greater momentum resulting in greater destructive capacity. Mixtures with a higher percentage 
of hydrogen have increased thermal conductivity in the range of $3000 \mathrm{~K}-5000 \mathrm{~K}$ and $~ 10,000$ $\mathrm{K}-18,000 \mathrm{~K}$, which may also allow for more energy to be transferred to the MSW (Figure 23).

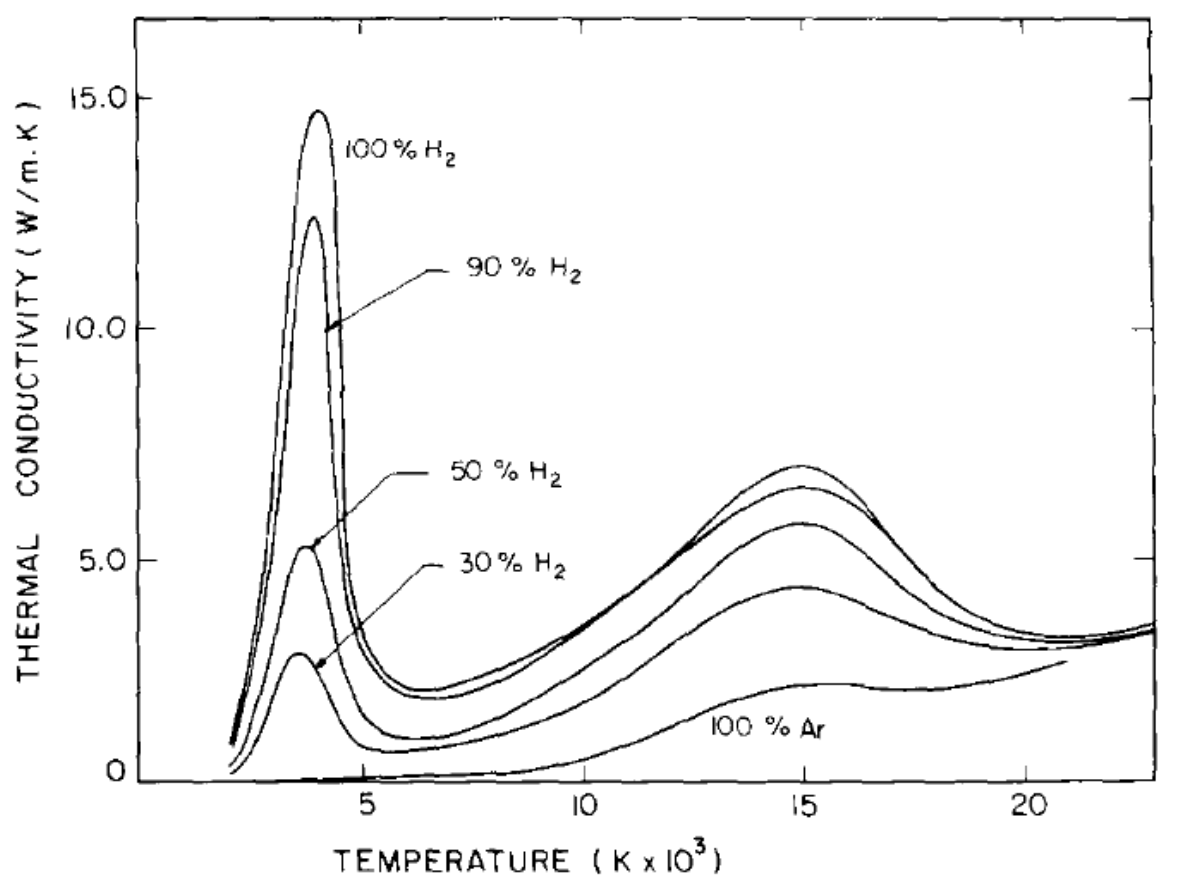

Figure 23: Thermal conductivity for $\mathrm{Ar} / \mathrm{H}_{2}$ mixtures as a function of temperature ${ }^{[44]}$.

Nitrogen gas and the mixture of argon and hydrogen were both tested inside and out of the gasification chamber. Inside the chamber, the nitrogen melted the glass, iron and stainless steel faster. Outside of the chamber, the shapes and lengths of the plasma showed that the argon/hydrogen mix made a shorter plasma plume (Figure 24). The argon/hydrogen plasma was also at an angle off the central axis of the torch, which must have been caused by the vortex generator melting. This test between the two gases was not conclusive due to the failure of the vortex generator. The regulator on the hydrogen tank was also leaking at the time, so the exact mixture was impossible to determine. 


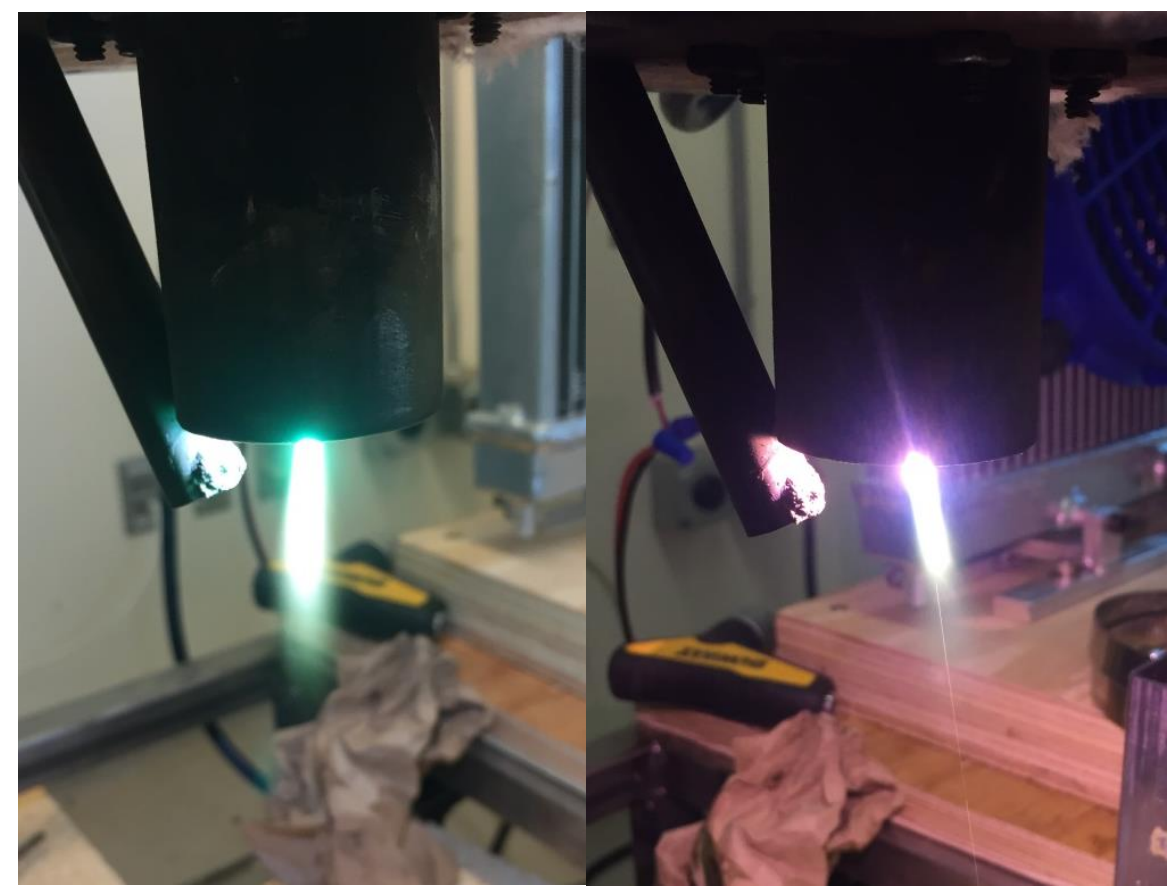

Figure 24: Plasma produced with compressed nitrogen (left) and argon/hydrogen mixture (right).

\section{$\underline{5.3 \text { Gasification chamber }}$}

The design of the gasification chamber is similar to a blast furnace, with the plasma entering the chamber in the center of the cylinder and the exhaust gases exiting along the perimeter. The chamber is meant to hold some volume of molten metal and glass as a heat mass to reduce the amount of energy input required to break down the incoming material.

The chamber was made by forming a sheet of 0.062 -inch carbon steel sheet into an 8-inch diameter, 10-inch high cylinder and welding a flat sheet to the base. A piece of 1-inch thick kaolin wool was placed at the bottom of the chamber and Mizzou castable refractory cement was then poured in the cylinder to form a base with a depth of approximately 2 inches. A 3-inch diameter round steel bar with a piece of cardboard wrapped around it was then placed in the center of the chamber and the outer edge of the chamber which was lined with 1-inch thick kaolin wool (Figure 25). The gap between the kaolin wool and the steel cylinder was filled with the refractory cement and vibrated slightly by hitting the outside of the chamber with a steel rod. The refractory cement was added until it came over the edge of the kaolin wool to form a nearly sealed mass. The outer edge at the top of the cylinder was blocked off with bent pieces of cardboard to allow the cured solid some room for thermal expansion (Figure 25). 


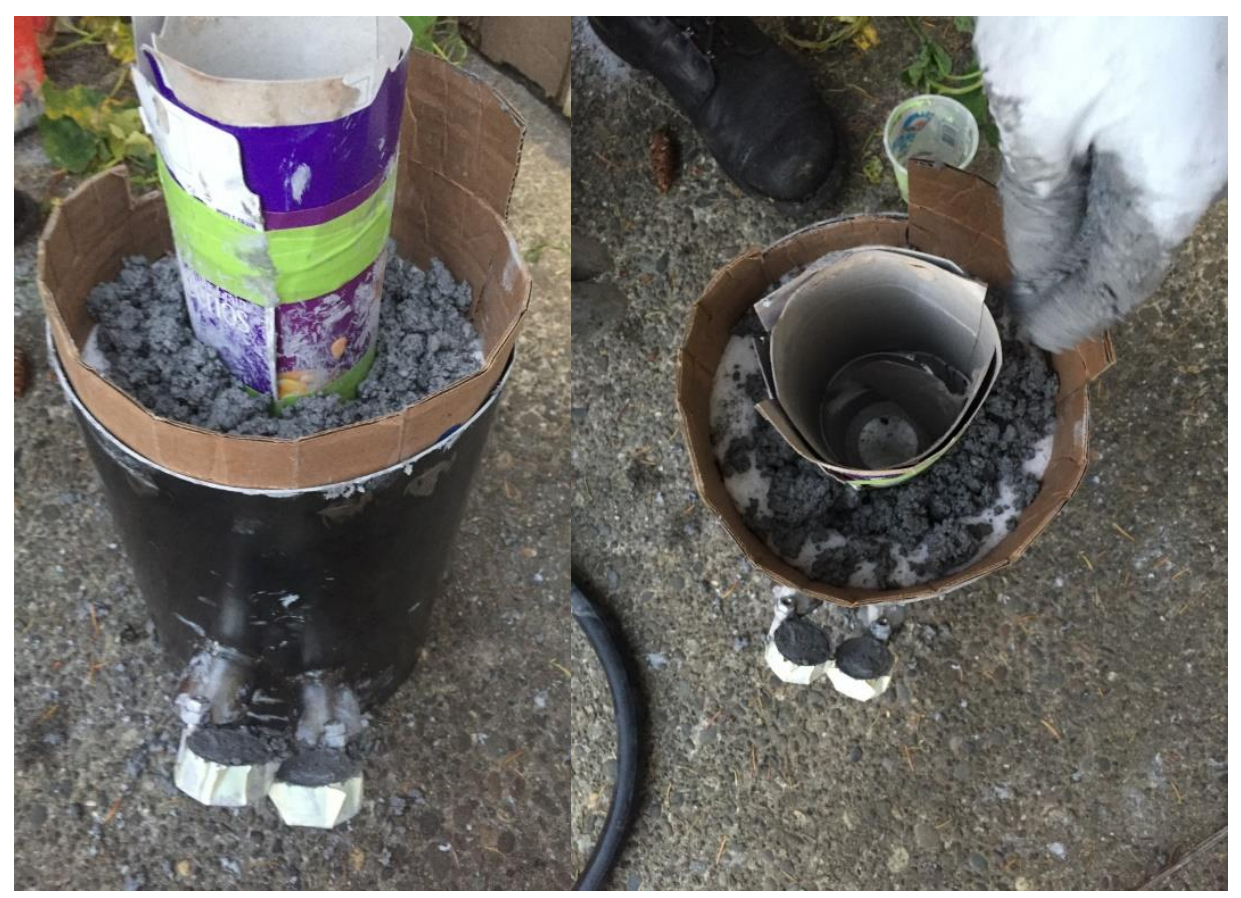

Figure 25: Gasification chamber being filled with refractory cement.

There are two offset holes at different heights from the base to allow excess molten glass and metals to be removed. Each of these holes has a tube filled with refractory material with a 0.375inch channel for material passage. The ends of each of these tubes have a valve made from filling a short section of tubing with refractory material and attaching it to the outlet tube with an offset bolt to allow for rotation when opening and closing.

The top of the gasification chamber has a flange welded on with holes for bolting the top plate in place. A gasket cut from automotive exhaust gasket is used to seal between the plate and the flanges and is held together using 1/4-20 bolts. A layer of 1-inch thick kaolin wool is placed on the top of the chamber and aligned with the parts protruding from the top plate before it is closed (Figure 26). The top plate has a half-inch diameter inlet tube protruding out (left side) for feeding test material into the gasification chamber. The exhaust is a welded stainless steel tube (right side). There is also a view port with a round quartz window to view the inside of the chamber as it is running. The torch centering device has three welded feet on the top plate to bolt the device in place. The center of the top plate has a large hole for the cooling jacket of the torch to slide into and holes for the cooling torch flange to be bolted. 


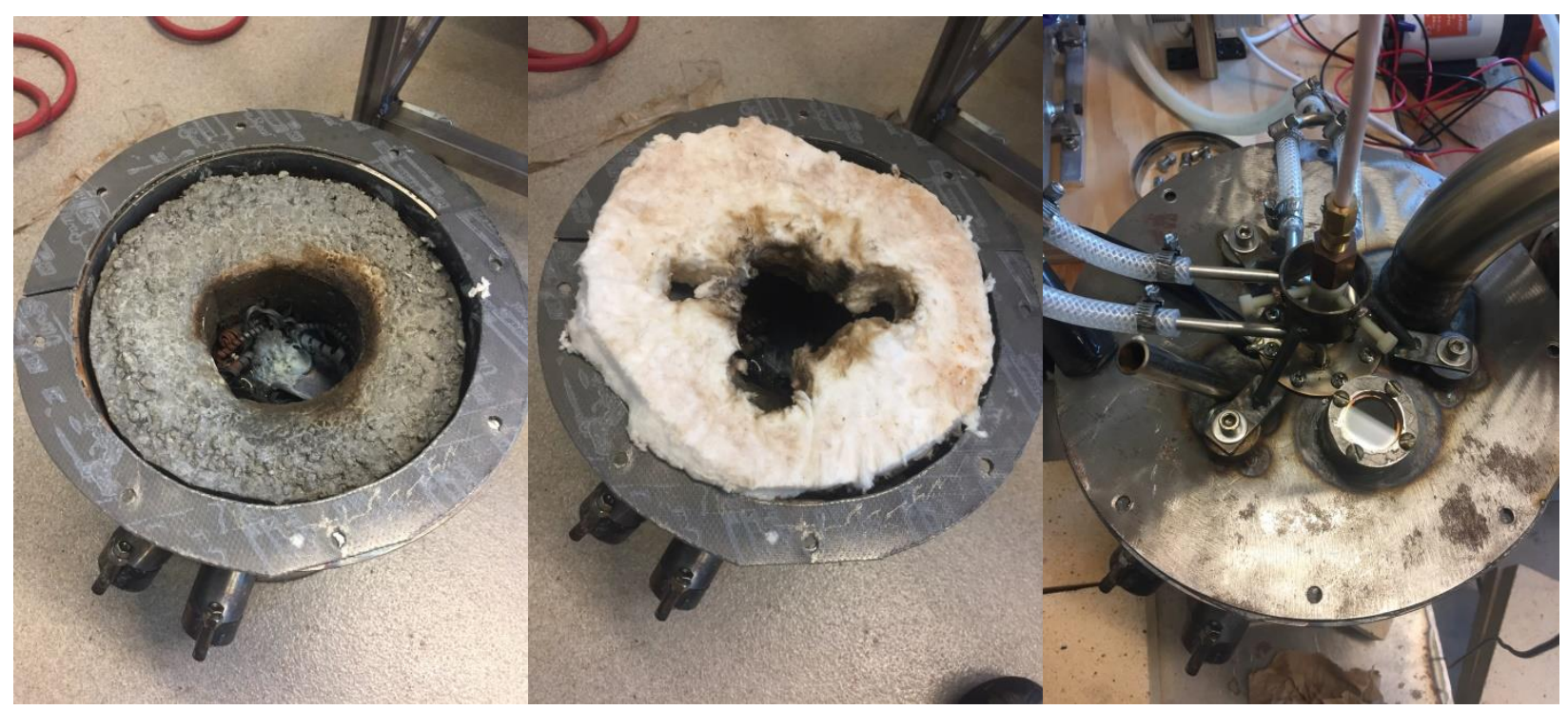

Figure 26: Gasification chamber filled with test material of steel, stainless steel, aluminum, copper scraps, and glass with the gasket in place (left); with the kaolin wool insulation in place (middle); and the top plate.

\section{$\underline{5.4 \text { Cyclone Particle Separator }}$}

The cyclone particle separator was designed based on typical high efficiency dimensions from the Air Pollution Control Technology Handbook ${ }^{[22]}$. The dimensions of the inlet and outlet were chosen based on the most easily accessible steel tubing, with the dimensions of fabricated parts based on the inlet and outlet. The inlet, outlet, and dust collection container are all connected with welded flanges for easy assembly and maintenance (Figure 27). The outlet tube is welded to a conical reducer, which was ground down to fit between the 1.75-inch diameter tubing and the 2-inch bend to the next treatment process.

\section{$\underline{5.5 \text { Catalytic converter }}$}

A catalytic converter was purchased to be used after the particle separator. Each end had flanges welded on to connect to the prototype system tubing (Figure 27). The catalytic converter used is rated for the exhaust of a 5.7L internal combustion engine. This catalytic converter will likely not be running at peak efficiency, as the gasification of MSW is not likely to be consistent in the amount of $\mathrm{NO}_{\mathrm{x}}$ or $\mathrm{CO}$ that produced in the exhaust. The catalytic converter is placed before the scrubber to allow the oxidation and reduction reactions that are catalyzed to occur at high temperature. The scrubber greatly reduces the temperature of the syngas. 


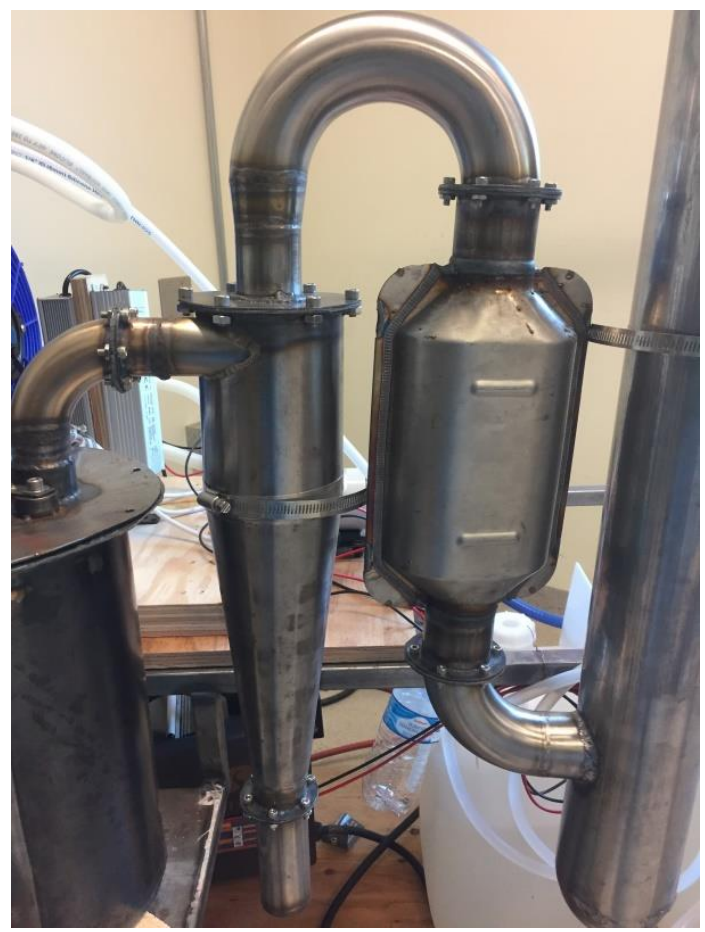

Figure 27: Cyclone particle separator and catalytic converter.

\subsection{Scrubber}

The chosen scrubber design was a packed column scrubber, composed of a stainless steel pipe with course polymer filter media (Figure 28). Ten to fourteen layers of 2-inch thick course polymer filter media are stacked inside for increased surface area. At the top of the scrubber is a laser cut plate with a mist nozzle mounted in the center where the scrubbing liquid is sprayed in, and four large openings for the exhaust to continue through the system. The mist settles and drips down the filter media as the gases injected from the base push through toward the top of the scrubber. The exhaust from the scrubber comes out the top while the scrubber liquid pools at the base and exits through a tube into the scrubber reservoir. Above the mist nozzle is a mist collector, which is several layers of stainless steel mesh, which captures droplets and allows them to combine and drip back down into the scrubber to reduce liquid losses.

The scrubber liquid is stored in two 5-gallon polyethylene jugs connected using three 1-inch bulkheads. The inlet of each jug has a polyethylene sheet placed vertically approximately $1 / 3$ of the way down into the jug that acts as a baffle to reduce turbulence from the incoming liquid. Reducing the turbulence would allow particles to settle out faster. Smaller particles may not settle in the short distance provided by these two jugs. There is a 36 Series SEAFLO diaphragm 
pump pumping the scrubber liquid out of the scrubber reservoir and into the scrubber through the mist nozzle. The liquid is pumped through silicone tubing.

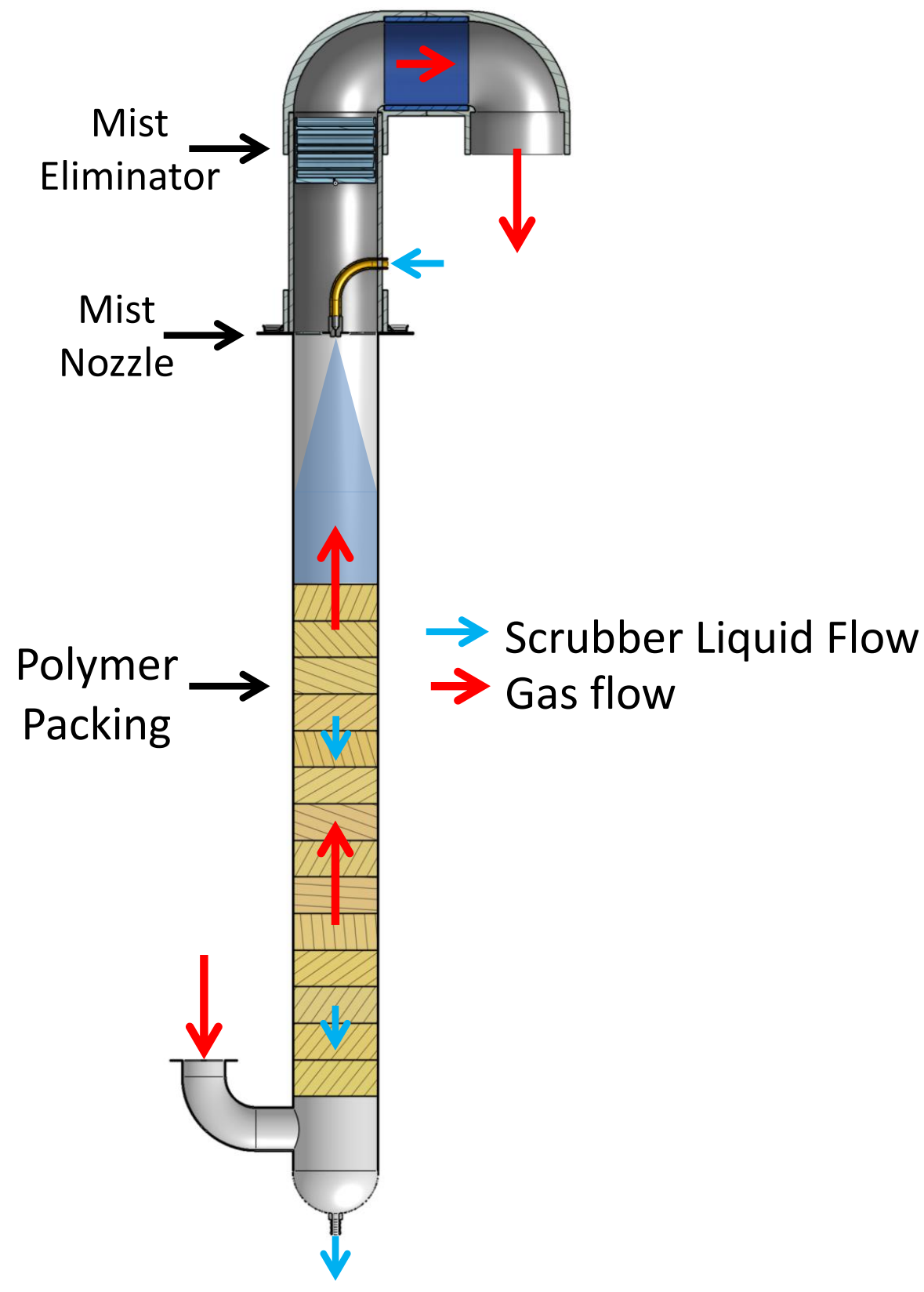

Figure 28: Packed column scrubber schematic.

The scrubber liquid used is a $0.003 \mathrm{~mol} / \mathrm{L}$ solution of sodium hydroxide $(\mathrm{NaOH})$ in deionized water. This initial $\mathrm{pH}$ was 11.48 , with the expectation of the $\mathrm{pH}$ dropping as it equilibrates with the carbon dioxide in the air, and then continues to drop as the exhaust gas flows through the scrubber. Sodium hydroxide was used as a 50\% solution was readily available in the lab. The solution in the scrubber was going to be mixed to a $\mathrm{pH}$ of 9.0 with calcium hydroxide instead of 
sodium hydroxide as it is less hazardous. If the $\mathrm{pH}$ is held below 9.0, there will be less $\mathrm{CO}_{2}$ absorbed into the scrubber liquid, which will allow for more of the target pollutants to be absorbed instead $^{[22]}$.

\section{$\underline{5.7 \text { Sensors }}$}

The sensors used on this prototype are ones that are low cost and readily available. This prototype uses four sensors made by SPEC sensors detecting $\mathrm{CO}, \mathrm{CO}_{2}, \mathrm{SO}_{2}, \mathrm{NO}_{2}, \mathrm{NH}_{3}$, and $\mathrm{H}_{2} \mathrm{~S}$. A BME 680 sensor is used to measure temperature, pressure, humidity and the resistance of the gas. There is also a Plantower PM2.5 sensor, and a D3231 Precision RTC Breakout made by Adafruit used to record the date and time of measurements. All of these sensors are connected to an Arduino Due using a breadboard and some 10kohm and 100kohm resistors. All of the measurements are displayed on a 1.8" TFT Display Breakout LED screen also made by Adafruit (Figure 29). These sensors were calibrated by placing them in a container filled with nitrogen gas and adjusting the code to match the electrical signal recorded while in this container. Python code is used to record the data in comma separated variable files as the data is recorded.

These sensors were placed in the syngas stream before the tube sucking the syngas into the fume hood to measure any components that were not removed from the syngas. The piping for the syngas leaving the prototype is 4-inch interior diameter and the sensors can be placed inside without modification. When the system was first turned on, the sensors recorded for the first 30 seconds and then all stopped sending a signal. This failure of all the sensors is likely due to the velocity of the gas across sensors that were designed for ambient air. The PM2.5 particle sensor stopped working altogether after the initial test and was apparently damaged by the high velocity. To reduce the velocity of the syngas exiting the prototype, a larger cross sectional area is required between the exit and the fume hood.

\section{$\underline{5.8 \text { Power Supply }}$}

There are several power supplies used in this prototype. The power supply for the plasma torch is a LOTOS LTP5000D non-touch pilot arc plasma cutter. The cables that came with this plasma cutter were cut and attached in the appropriate places to the plasma torch that was designed and built for this prototype. This was an inexpensive and simple way to generate the 


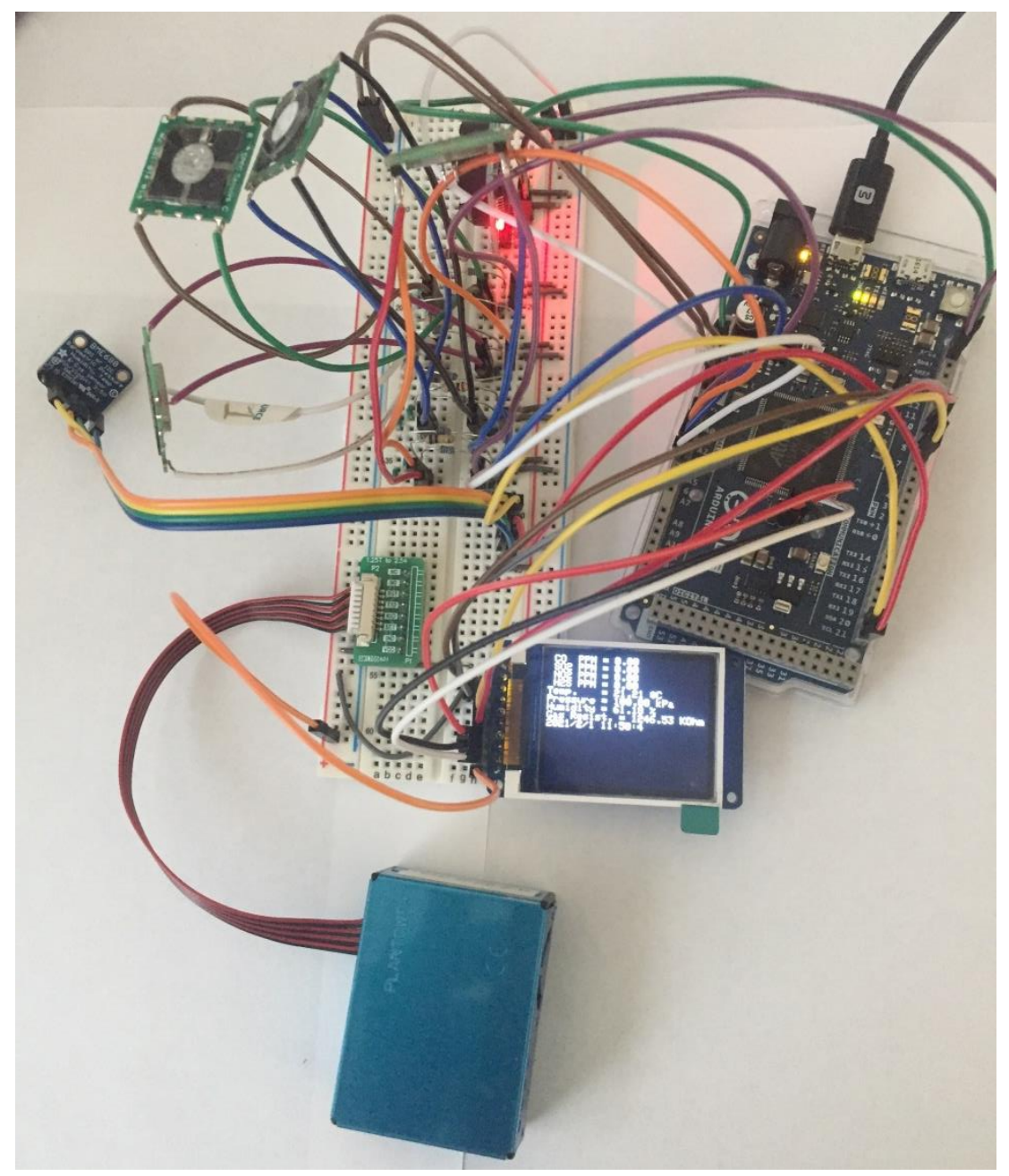

Figure 29: Sensors, Arduino controller and LCD screen.

correct current and amperage for the plasma torch to operate. The gas line was connected to the fitting that holds the electrode collet and the negative terminal is also connected to this fitting, along with the non-touch pilot connection. The ground of the power supply is connected to the cooling jacket of the torch. This power supply provides the initial spark from the non-touch pilot arc and automatically adjusts the voltage and current from startup to normal operating conditions. It can run on both $110 \mathrm{v}$ and $220 \mathrm{v}$ and can be operated with any gas. There are also two $120 \mathrm{v}$ AC to $12 \mathrm{v}$ DC converters on the prototype to run the pumps and sensor system.

\section{$\underline{5.9 \text { Frame }}$}

The frame is built with 1-inch $\mathrm{x}$ 1-inch hollow square tubing with a platform for the gasification chamber, scrubber reservoir and power supply, and the torch cooling system. The 
whole system is relatively small and has a footprint of 36 inches by 48 inches and is just over 66inches tall. The cyclone, catalytic converter and scrubber are mounted on some tubes that were welded vertically a few inches offset from the horizontal center support tube (Figure 30). The entire frame was put on caster wheels to easily move around the lab. On the platform with the gasification chamber, there are several refractory bricks for the gasification chamber to be mounted. The wiring for the torch, pumps and sensors is run along the central support tube and along other tubes in the frame. The switches for turning on each of the pumps, the torch and the sensors are mounted to a laser cut sheet of steel welded to the top right corner of the front of the frame.
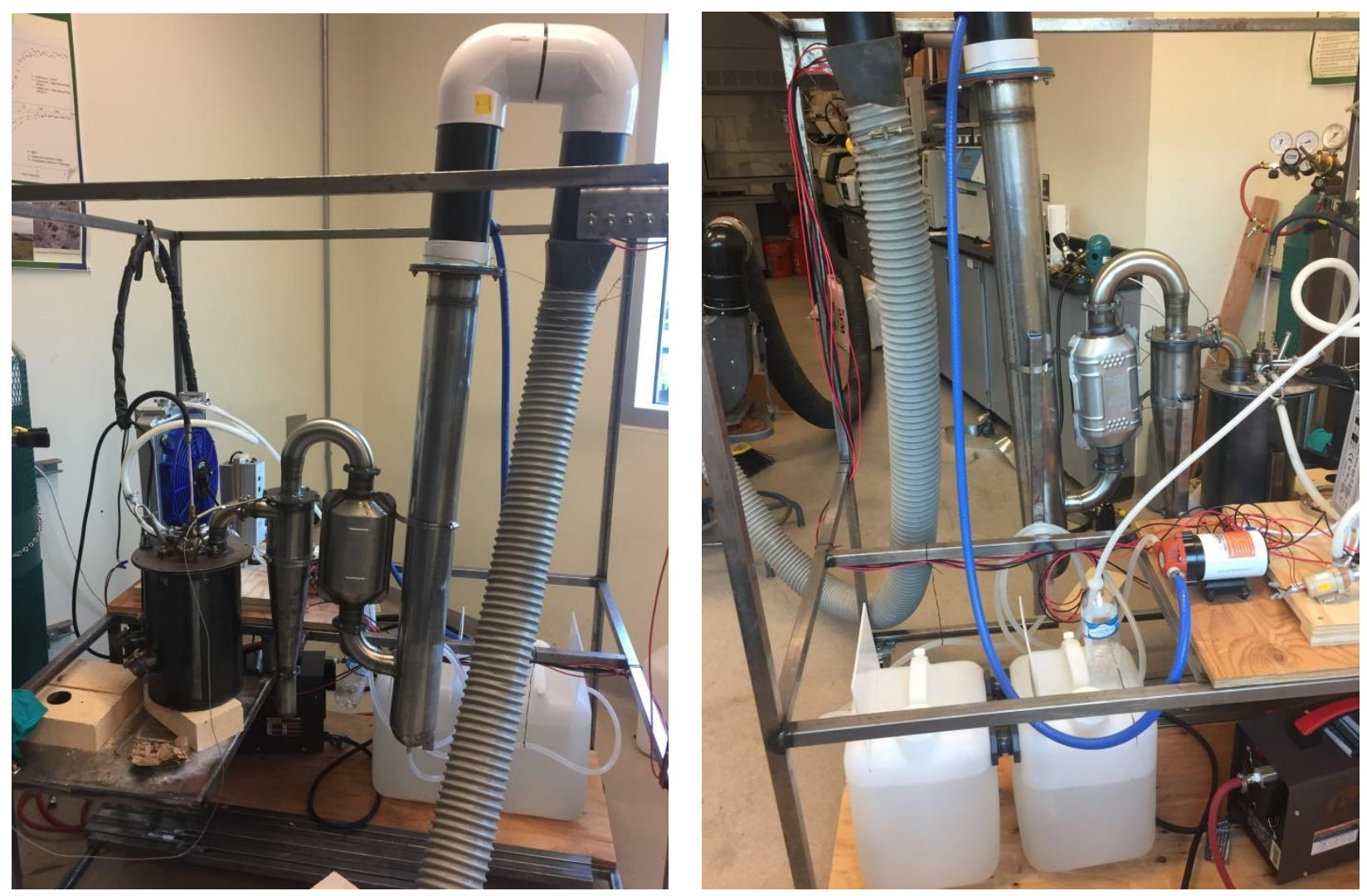

Figure 30: Plasma system prototype frame front (left) and back (right).

\subsection{System Testing}

The materials used for melting tests included copper, aluminum, glass, carbon steel and stainless steel. Small pieces of aluminum, with a melting temperature of $660{ }^{\circ} \mathrm{C}$, melted easily, while the larger pieces of approximately 1-inch thickness took several minutes due to the high 
thermal conductivity of the metal. Much of this heat was transferred to the other material below in the gasification chamber. Copper wire melted quickly, with a melting point of $1084{ }^{\circ} \mathrm{C}$. Small pieces of glass melted quickly and formed a pool of molten material, which was the goal for the inorganic material tests (Figure 31). Glass melts between $1400{ }^{\circ} \mathrm{C}$ and $1600{ }^{\circ} \mathrm{C}$ and is an insulating material so there is less heat being transferred to the surrounding material when direct heat is applied. Small pieces of carbon steel, with melting temperature between $1425{ }^{\circ} \mathrm{C}$ and $1540{ }^{\circ} \mathrm{C}$, melted slowly, and pieces of stainless steel with a 1/4-inch thickness took longer. During this test with the stainless steel, the vortex generator melted and the electrodes became misaligned, greatly reducing the size of the plasma plume.

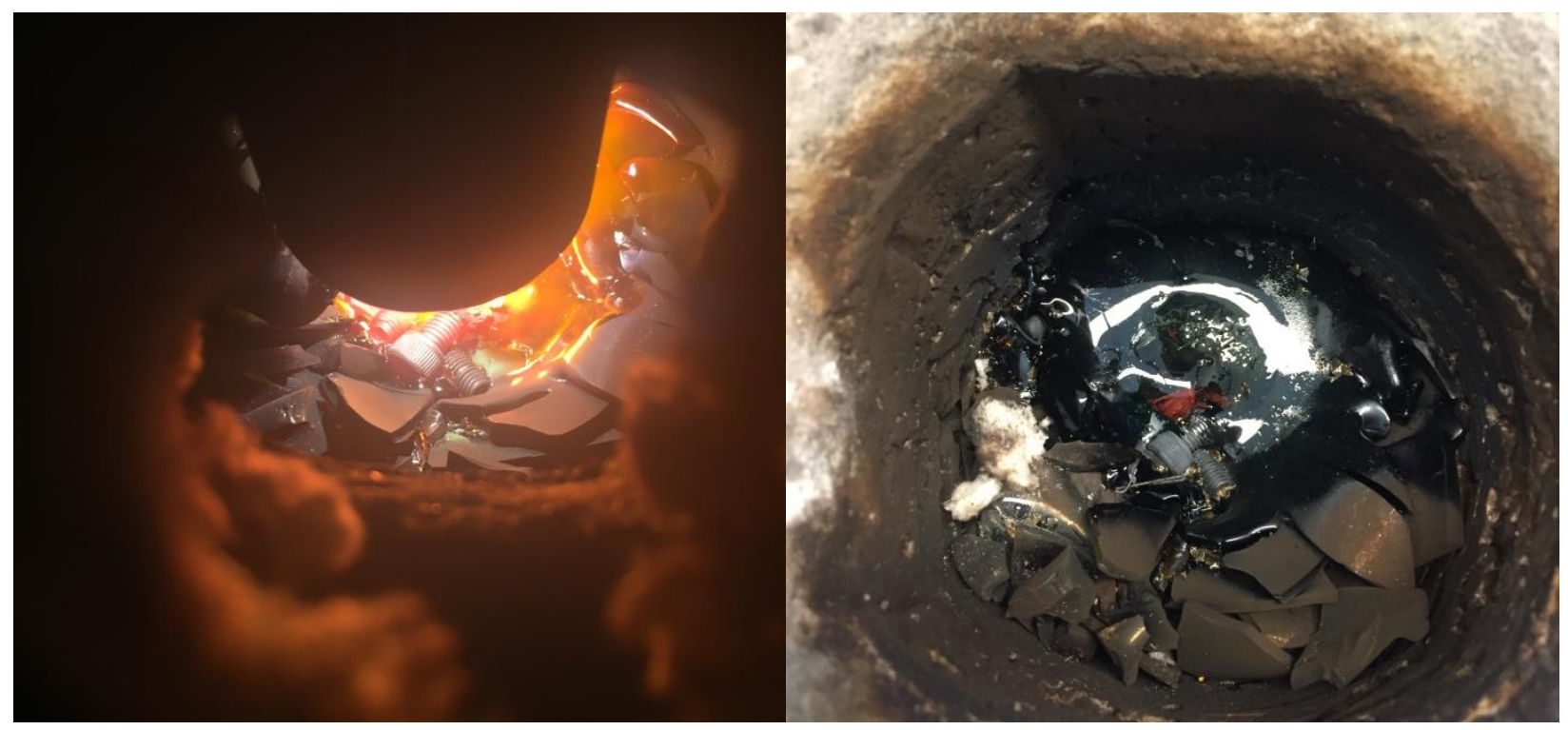

Figure 31: Molten glass and metals inside the gasification chamber. 


\subsection{DISCUSSION AND CONCLUSION}

The goal of this project was to develop a prototype plasma gasification system to treat municipal solid waste (MSW) with a design aimed at minimizing regulated emissions in a footprint small enough to be transported allowing waste management directly at the source. A small-scale plasma gasification system was designed and built with emission controls in place. The built system is contained in a package small enough to fit on the back of a standard pickup truck. Overall, this project met its goals of a working small scale plasma gasification system with emissions controls that can be transported. However, the gas cylinders required to produce plasma would also need transport and the built system requires both a 220v circuit and a $110 \mathrm{v}$ circuit to work. While the built system has been tested, the emission controls incorporated in this pilot-scale design will benefit from additional research.

The plasma torch built for this prototype did not produce a plasma plume large enough to fully break down the materials added to the chamber. Part of the failure is due to a lack of sufficient power to the torch. With more power than that of a $220 \mathrm{v}, 20$ amp circuit available in the PSU lab, the torch would be able to increase the degree of ionization of the passing carrier gas. This is a prototype plasma gasification system, though the plasma torch and gasification chamber were not matched in capacity. This torch may be improved with many more hours of testing and nozzle designs, which was not the main focus of the project.

This project currently has several consumables and power requirements that would limit its application in many parts of the world. The compressed gas that the torch runs on must be reasonably pure nitrogen, a mixture of argon and helium, or carbon dioxide. These compressed gases are not available in many of the places of the world that do not have waste management systems. The plasma torch design for this project was meant to run on compressed air, which may have been ambitious.

With minimal electrical engineering and plasma torch background, there were many challenges with this project. The plasma torch is far from optimal, yet is still a far cheaper option than commercially available plasma torches that are built for similar purposes. Much of the testing required in the project was getting the torch to work enough to continue with other aspects of the project. After researching more about plasma torches that are used in industry, the problems of electrodes and process gases could be greatly simplified by using steam instead. 
The 110 volt and 220 volt power requirements would also prevent this system from being usable in many parts of the world, unless there was some power generation and storage built into the package for startup, such as wind turbines or solar cells along with a battery pack. The scrubber system also has chemical requirements to keep the $\mathrm{pH}$ high enough to clean the exhaust that may be unavailable in other parts of the world.

The emission control components used are not expected to efficiently treat or capture $\mathrm{CO}$ or $\mathrm{CO}_{2}$. With a varying fuel to air ratio, the catalytic converter will not be optimally oxidizing $\mathrm{CO}$ and will likely let much of this CO pass by unoxidized. The scrubber is not expected to absorb much of this $\mathrm{CO}_{2}$, as there does not seem to be a long enough residence time in the scrubber for the volume of exhaust gases produced. The solubility of these gases in water also decreases with increasing temperature, further reducing the capacity for absorption into the scrubber liquid. Without a heat exchanger in place before the scrubber, the exhaust gases to be treated will likely increase the temperature of the scrubber liquid by a fair amount. The effectiveness of the scrubber still needs to be tested to verify whether it can handle treating the volume of exhaust gas produced. It is expected that the scrubber and scrubber reservoir size should be increased to meet the treatment requirements based on the flow volume of exhaust gases.

As the scrubber in this system removes pollutants from the exhaust gas, it will build up these compounds in the scrubber reservoir. Removal of these compounds has not been included in this project, and is an important part of treatment. These compounds can be separated through further processing. Removal and recovery of materials from the exhaust gases are necessary to make the plasma gasification process desirable compared to pyrolysis or incineration waste reduction processes.

The way municipal solid waste is currently dealt with is not sustainable. Much of the MSW is buried to slowly decay and release pollutants that must be remediated for years to come. The processes used today to reduce the volume of this waste and generate power still cause environmental issues that need to be considered. Plasma gasification is a promising solution that still needs optimization, yet is able to greatly reduce the volume of waste that is produced while providing the opportunity for material recovery and energy generation. With recycling, composting and re-use of materials, plasma gasification can be used to account for all the other materials that have reached the end of their useful life. With the addition of gas separation, fuel cells for energy production from the hydrogen gas, a heat recovery system, and material recovery 
systems applied to the syngas, this technology has the potential to change the way society deals with waste. Plasma gasification of MSW only needs people to focus their time and resources to optimize the technology and focus on the future of how waste is treated. This technology can help the economy go from a cradle to grave perspective to a cradle to cradle perspective.

Small-scale systems like the one built for this project could be built all around the world to treat waste where it is produced to reintroduce raw materials back into the economy. The more small systems that are built, the shorter waste collection vehicles need to travel and fewer emissions will be produced. Small-scale systems also have a far lower capital cost than the metropolitan sized processing plants, which would allow them to be built in a shorter period of time. When plasma gasification systems are built on mobile platforms, the plasma systems could be used for disaster cleanup or to help those parts of the world with no waste management systems in place to reduce their pollution. 


\subsection{FURTHER RESEARCH}

After doing more research, it was discovered that many of the newest plasma torches use steam as the carrier gas, which can greatly increase the life of the electrodes, along with other benefits ${ }^{[45],[46],[47]}$. Using steam eliminates the need for any compressed gas cylinders to be stored close by, which allows the system to be used in more parts of the world. Water vapor also does not contain nitrogen, which greatly reduces the amount of nitrogen oxides that could form and could eliminate the need for $\mathrm{NO}_{\mathrm{x}}$ emission controls ${ }^{[48]}$. Injecting steam into the gasification chamber has been shown to increase the efficiency of breakdown of MSW in large-scale systems currently in place ${ }^{[49],[50]}$. A steam plasma torch would require a source of heat to vaporize the water, which would increase the power consumption. If a heat exchanger were used to capture heat from the exhaust gases to help preheat the steam, it would reduce this extra power requirement. This preheated steam would also slightly reduce the power required to form the plasma. The steam would also act as a heat sink for the torch, causing a self-cooling effect, which would eliminate the need for an external cooling system. Water has a high heat capacity, so if the steam were continuously fed into the system above $100^{\circ} \mathrm{C}$, it would be constantly absorbing any excess heat above that temperature. The pressure of the steam as it is produced can be regulated just as a compressed gas would, so there would be no issue of decreasing gas pressure from a compressed gas tank as the supply runs low. Based on the benefits of steam plasma over compressed air plasma, it is recommended that the next prototype plasma torch be designed for use with steam.

The next steps for this small scale plasma gasification system would be to test various materials to determine the effectiveness of the emission controls. The emissions testing plan could start with wood pellets as a surrogate for organic material. These tests could be done by feeding in a known mass of wood pellets into the gasification chamber and recording the concentration of $\mathrm{CO}, \mathrm{CO}_{2}, \mathrm{NO}_{2}, \mathrm{NH}_{3}, \mathrm{SO}_{2}$, and $\mathrm{H}_{2} \mathrm{~S}$ for the duration of the test. The mass of each of these components could then be found by integrating the plot of concentration vs. time. The $\mathrm{pH}$ of the scrubber solution should also be recorded before and after each test. Small samples of syngas should be taken from each test and analyzed using gas chromatography to determine the compounds that made it through the emissions controls. This procedure should be repeated with many more materials to simulate the composition of MSW. Tests should start with 
simple, non-toxic materials and continue with increasing material complexity and toxicity to test how well the emissions system could handle refining the exhaust gases produced. 


\subsection{REFERENCES}

1. Hoornweg, D., Bhada-Tata, P. 2012. What a Waste: A Global Review of Solid Waste Management. Urban development series; knowledge papers no. 15. World Bank, Washington, DC. @ C World Bank. https://openknowledge.worldbank.org/handle/10986/17388 License: CC BY 3.0 IGO.

2. Jaunich, M. K., James, L. W., Decarolis, J. F., Gaston, E. V., Barlaz, M. A., Bartelt-Hunt, S. L., Jones, E. G., Hauser, L., Jaikumar, R. "Characterization of municipal solid waste collection operations." Resources, Conservation and Recycling 114 (2016): 92-102.

3. Nonroad Compression-Ignition Engines: Exhaust Emission Standards. EPA-420-B-16-022. United States, Environmental Protection Agency, Office of Transportation and Air Quality. March 2016.

4. Light-Duty Vehicles, Light-Duty Trucks, and Medium-Duty Passenger Vehicles: Tier 2 Exhaust Emission Standards and Implementation Schedule. EPA-420-B-17-028. United States, Environmental Protection Agency, Office of Transportation and Air Quality. September 2017.

5. Peri, G., Ferrante, P., La Gennusa, M., Pianello, C., Rizzo, G. "Greening MSW management systems by saving footprint: The contribution of the waste transportation." Journal of environmental management 219 (2018): 74-83.

6. Pérez, J., Lumbreras, J., Rodriguez, E., Vedrenne, M. "A methodology for estimating the carbon footprint of waste collection vehicles under different scenarios: Application to Madrid." Transportation Research Part D: Transport and Environment 52 (2017): 156-171.

7. Economopoulou, M. A., Economopoulou, A. A., Economopoulos, A. P. "A methodology for optimal MSW management, with an application in the waste transportation of Attica Region, Greece." Waste management 33.11 (2013): 2177-2187.

8. Das, S., and Bhattacharyya, B. K. "Optimization of municipal solid waste collection and transportation routes." Waste Management 43 (2015): 9-18.

9. Chai, J., Miura, N. 2002. Comparing the performance of landfill liner systems. J Mater Cycles Waste Management. 4, 135-142.

10. Henken-Mellies, W. U., Zanzinger, H., Gartung, E. "Long-term field test of a clay geosynthetic barrier in a landfill cover system." Clay Geosynthetic Barriers (2002): 303-309. 
11. Hirshfeld, S., Vesilind, P., Pas, E. 1992. Assessing the True Cost of Landfills. Waste Management \& Research. December 1992, Vol. 10, Iss. 6, 471-484.

12. Fatta, D., Papadopoulos, A., Loizidou, M. 1999. A study on the landfill leachate and its impact on the groundwater quality of the greater area. Environmental Geochemistry and Health, 21, 175-190.

13. Rathje, William L., Hughes, W. W., Wilson, D. C., Tani, M. K., Archer, G. H., Hunt, R. G., Jones, T. W. "The archaeology of contemporary landfills." American Antiquity (1992): 437447.

14. United States, Environmental Protection Agency. "Energy Recovery from the Combustion of Municipal Solid Waste (MSW)" 31 July, 2020. https://www.epa.gov/smm/energy-recoverycombustion-municipal-solid-waste-msw.

15. Sowards, N., d'Alene, C. Low Pollution Incineration of Solid Waste. June 30, 1975. US Patent 4,060,041. Google Patents, https://patentimages.storage.googleapis.com/76/b2/bf/b9c9137c0f69e5/US4060041.pdf. Accessed 25 Oct. 2020.

16. Garrett, D., Mallan, G. Pyrolysis Process for Solid Wastes. May 17, 1976. US Patent 4,153,514. Google Patents, https://patentimages.storage.googleapis.com/b2/97/ad/70ce2fbcf7e8b7/US4153514.pdf. Accessed 25 Oct. 2020.

17. Qi, Z., Jie, C., Tiejun, W., Ying, X. 2006. Review of biomass pyrolysis oil properties and upgrading research. Energy Conversion and Management 48 (2007) 87-92.

18. Conesa, J.A., Font, R., Fullana, I., Martín-Gullón, I., Aracil, A., Gálvez, A., Moltó, J., Gómez-Rico, M.F. Comparison between emissions from pyrolysis and combustion of different wastes. Journal of Analytical and Applied Pyrolysis, 84 (2009) 95-102.

19. Danthurebandara, M., Van Passel, S., Vanderreydt, I., Van Acker, K. 2015. "Environmental and economic performance of plasma gasification in Enhanced Landfill Mining”. Waste Management Vol. 45, 458-467.

20. Janajreh, I., Shabbar Raza, S., Snaer Valmundsson, A. 2013. "Plasma gasification process: Modeling, simulation and comparison with conventional air gasification”. Energy Conversion and Management. Vol 65, 801-809. 
21. Scott, D. A., Kovitya, P., Haddad, G. N. 1989. "Temperatures in the plume of a de plasma torch”. Journal of Applied Sciences. Vol 66. 5232-5239.

22. Schnelle, K., and Brown, C. Air Pollution Control Technology Handbook. Boca Raton, CRC Press LLC, 2002.

23. Srivastava, R. K., Hall, R. E., Khan, S., Culligan, K., Lani, B. W. "Nitrogen oxides emission control options for coal-fired electric utility boilers." Journal of the Air \& Waste Management Association 55.9 (2005): 1367-1388.

24. Pankow, J. F. Aquatic Chemistry Concepts. CRC Press, 2020, p. 186-187.

25. Harling, Alice M., Glover, D., Whitehead, C., Zhang, K. "The role of ozone in the plasmacatalytic destruction of environmental pollutants." Applied Catalysis B: Environmental 90.12 (2009): 157-161.

26. Batakliev, T., Georgiev, V., Anachkov, M., Rakovsky, S., Zaikov, G. E. "Ozone decomposition." Interdisciplinary toxicology 7.2 (2014): 47-59.

27. Catalytic Converters. Construction. https://www.catalyticconverters.com/construction/. 2020. Accessed 13 January 2021.

28. Pankow, J. F. Air Pollution in the Troposphere (where the bad ozone lives). February 2020. PowerPoint Presentation.

29. United States, US EPA. Control Techniques for Carbon Monoxide Emissions, June 1979. January 2021.

30. Srivastava, R. K., Jozewicz, W., Singer, C."SO2 scrubbing technologies: a review." Environmental Progress 20.4 (2001): 219-228.

31. Vallero, D. A. Fundamentals of air pollution. Academic press, 2014.

32. Cecala, A. B., O’Brien, A. D., Schall, J., Colinet, J. F., Fox, W. R., Franta, R. J., Joy, J., Reed, W. R., Reeser, P., W., Rounds, J. R., Schultz, M. J. "Dust control handbook for industrial minerals mining and processing." (2012).

33. "CFD Modelling Case Studies.” Computational Fluid Dynamics Consultancy | CFD | - Fluid Comp, thefluidcomp.com/projects.html.

34. Little, P., Wiffen, R. D. "Emission and deposition of lead from motor exhausts-II. Airborne concentration, particle size and deposition of lead near motorways." Atmospheric Environment (1967) 12.6-7 (1978): 1331-1341. 
35. Chiaradia, M., François C. "Behaviour of airborne lead and temporal variations of its source effects in Geneva (Switzerland): comparison of anthropogenic versus natural processes." Atmospheric Environment 34.6 (2000): 959-971.

36. Mukai, H., Furuta, N., Fujii, T., Ambe, Y., Sakamoto, K., Hashimoto, Y. "Characterization of sources of lead in the urban air of Asia using ratios of stable lead isotopes." Environmental Science \& Technology 27.7 (1993): 1347-1356.

37. Froines, J. R., Baron, S., Wegman, D. H., O’Rourke, S. "Characterization of the airborne concentrations of lead in US industry." American Journal of Industrial Medicine 18.1 (1990): $1-17$.

38. Spear, T. M., Svee, W., Vincent, J. H., Stanisich, N. "Chemical speciation of lead dust associated with primary lead smelting." Environmental Health Perspectives 106.9 (1998): $565-571$.

39. United States, Environmental Protection Agency. "Initial List of Hazardous Air Pollutants with Modifications.” June 18, 2020. https://www.epa.gov/haps/initial-list-hazardous-airpollutants-modifications . Accessed 13 January, 2021.

40. Van den Berg, M., Bimbaum, L., Bosveld, T. C., Brunstrom, B., Cook, P., Feeley, M., Giesy, J. P., Hanberg, A., Hasagawa, R., Kennedy, S. W., Kubiak, T., Larsen, J. C., Rolaf can Leeuwen, F. X., Djien Liem, A. K., Nolt, C., Peterson, R. E., Poellinger, L., Safe, S., Schrenk, D., Tillitt, D., Tysklind, M., Younes, M., Waern, F., Zacharewski, T. "Toxic equivalency factors (TEFs) for PCBs, PCDDs, PCDFs for humans and wildlife." Environmental health perspectives 106.12 (1998): 775-792.

41. Mukherjee, A., Debnath, B., Ghosh, S. D. "A review on technologies of removal of dioxins and furans from incinerator flue gas." Procedia Environmental Sciences 35 (2016): 528-540.

42. Mulholland, J. A., Akki, U., Yang, Y., Ryu, J. Y. "Temperature dependence of DCDD/F isomer distributions from chlorophenol precursors." Chemosphere 42.5-7 (2001): 719-727.

43. Das, S., Hazra, A., Banerjee, P. "PCDD/PCDFs: A Burden from Hospital Waste Disposal Plant; Plasma Arc Gasification Is the Ultimate Solution for Its Mitigation." Energy Recovery Processes from Wastes. Springer, Singapore, 2020. 9-21.

44. Boulos, M. I. "Thermal plasma processing." IEEE transactions on Plasma Science 19.6 (1991): 1078-1089. 
45. Uhm, H. S., Kim, J. H., Hong, Y. C. "Disintegration of water molecules in a steam-plasma torch powered by microwaves." Physics of plasmas 14.7 (2007): 073502.

46. "Water-stream electroarc plasma advantages." Plazarium Manufacture and Service of Plasma Devices, 2021, www.plazarium.com/en/news/technology/waste-disposalrecycling/steam-plasma-advantages/. Accessed 10 February, 2021.

47. "Steam Plasma Torch.” Plasma Air AG, 2019, www. https://plasmaair.de/en/steam-plasmaburner/. Accessed 10 February, 2021.

48. Zhang, Q., Dor, L., Biswas, A. K., Yang, W., Blasiak, W. "Modeling of steam plasma gasification for municipal solid waste." Fuel processing technology 106 (2013): 546-554.

49. Park, H. S., Kim, C. G., Kim, S. J. "Characteristics of PE gasification by steam plasma." Journal of Industrial and Engineering Chemistry 12.2 (2006): 216-223.

50. Alter NRG/Westinghouse. The Alter NRG / Westinghouse Plasma Gasification Process. Juniper Consultancy Services Limited, November 2008. http://energy.cleartheair.org.hk/wpcontent/uploads/2013/09/Westinghouse_Plasma_Gasification.pdf. 